\title{
THE MULTI-OBJECT, FIBER-FED SPECTROGRAPHS FOR THE SLOAN DIGITAL SKY SURVEY AND THE BARYON OSCILLATION SPECTROSCOPIC SURVEY
}

Stephen A. Smee ${ }^{1}$, James E. Gunn ${ }^{2}$, Alan Uomoto ${ }^{3}$, Natalie Roe ${ }^{4}$, David Schlegel ${ }^{4}$, Constance M. Rockosi ${ }^{5}$, Michael A. CarR ${ }^{2}$, French Leger ${ }^{6}$, Kyle S. Dawson $^{7}$, Matthew D. Olmstead $^{7}$, Jon Brinkmann $^{8}$, Russell Owen $^{6}$, Robert H. Barkhouser ${ }^{1}$, Klaus Honscheid ${ }^{9}$, Paul Harding $^{10}$, Dan Long ${ }^{8}$, Robert H. Lupton ${ }^{2}$, Craig Loomis $^{2}$, Lauren Anderson $^{6}$, James Annis ${ }^{11}$, Mariangela Bernardi ${ }^{12}$, VAishali Bhardwaj ${ }^{6}$, Dmitry Bizyaev ${ }^{8}$, Adam S. Bolton ${ }^{7}$, Howard Brewington $^{8}$, John W. Briggs ${ }^{13}$, Scott Burles ${ }^{14}$, JAmes G. Burns $^{9}$, Francisco Javier Castander ${ }^{15,16}$, Andrew Connolly ${ }^{6}$, James R. A. Davenport ${ }^{6}$, Garrett Ebelke $^{8}$, Harland Epps $^{5}$, Paul D. Feldman ${ }^{1}$, Scott D. Friedman $^{16}$, Joshua Frieman $^{11}$, Timothy Heckman ${ }^{1}$, Charles L. Hull $^{3}$, Gillian R. Knapp ${ }^{2}$, David M. Lawrence ${ }^{7}$, Jon Loveday ${ }^{17}$, Edward J. Mannery ${ }^{6}$, Elena Malanushenko ${ }^{8}$, ViKTOR Malanushenko $^{8}$, Aronne James Merrelli ${ }^{18}$, Demitri Muna ${ }^{19}$, Peter R. Newman ${ }^{8}$, Robert C. Nichol $^{20}$, Daniel Oravetz $^{8}$, Kaike Pan ${ }^{8}$, Adrian C. Pope ${ }^{21}$, Paul G. Ricketts ${ }^{7}$, Alaina Shelden $^{8}$, Dale Sandford $^{5}$, Walter Siegmund $^{6}$, Audrey Simmons ${ }^{8}$, D. Shane Smith ${ }^{9}$, Stephanie Snedden $^{8}$, Donald P. Schneider $^{22,23}$, Mark SubbaRao ${ }^{12}$, Christy Tremonti ${ }^{24}$, Patrick Waddell ${ }^{25}$, and Donald G. York ${ }^{26}$

${ }^{1}$ Department of Physics and Astronomy, Johns Hopkins University, Baltimore, MD 21218, USA; smee@ pha.jhu.edu

${ }^{2}$ Department of Astrophysical Sciences, Princeton University, Princeton, NJ 08544, USA

${ }^{3}$ Observatories of the Carnegie Institution of Washington, 813 Santa Barbara Street, Pasadena, CA 91101, USA

${ }^{4}$ Physics Division, Lawrence Berkeley National Laboratory, Berkeley, CA 94720, USA

${ }^{5}$ UC Observatories and Department of Astronomy and Astrophysics, University of California, Santa Cruz, 375 Interdisciplinary Sciences Building (ISB) Santa Cruz, CA 95064, USA

${ }^{6}$ Department of Astronomy, University of Washington, Box 351580, Seattle, WA 09195, USA

${ }^{7}$ Department of Physics and Astronomy, University of Utah, Salt Lake City, UT 84112, USA ${ }^{8}$ Apache Point Observatory, Sunspot, NM 88349, USA

${ }^{9}$ Department of Physics and Center for Cosmology and Astro-Particle Physics, Ohio State University, Columbus, OH 43210, USA

${ }^{10}$ Department of Astronomy, Case Western Reserve University, Cleveland, OH 44106, USA

${ }^{11}$ Fermi National Accelerator Laboratory, P.O. Box 500, Batavia, IL 60510, USA

12 Department of Physics and Astronomy, The University of Pennsylvania, 209 South 33rd Street, Philadelphia, PA 19104, USA

${ }^{13}$ HUT Observatory, Mittelman Family Foundation, P.O. Box 5320, Eagle, CO 81631, USA

${ }^{14}$ Physics Department, Massachusetts Institute of Technology, 77 Massachusetts Avenue, Cambridge, MA 02139, USA

${ }^{15}$ Institut de Ci encies de l'Espai (IEEC-CSIC), E-08193 Ballaterra, Barcelona, Spain

${ }^{16}$ Space Telescope Science Institute, Baltimore, MD 21218, USA

${ }^{17}$ Astronomy Centre, University of Sussex, Falmer, Brighton BN1 9QJ, UK

${ }^{18}$ Department of Astronomy, California Institute of Technology, Pasadena, CA 91125, USA

${ }^{19}$ Center for Cosmology and Particle Physics, New York University, 4 Washington Place, New York, NY 10003, USA

${ }^{20}$ Institute of Cosmology and Gravitation (ICG), Dennis Sciama Building, University of Portsmouth, Portsmouth PO1 3FX, UK

${ }^{21}$ High Energy Physics Division, Argonne National Laboratory, 9700 South Cass Avenue, Lemont, IL 60439, USA

22 Department of Astronomy and Astrophysics, The Pennsylvania State University, University Park, PA 16802, USA

${ }^{23}$ Institute for Gravitation and the Cosmos, The Pennsylvania State University, PA 16802, USA

${ }^{24}$ Department of Astronomy, University of Wisconsin-Madison, Madison, WI 53703, USA

${ }^{25}$ NASA Ames Research Center, Moffett Field, CA 94035, USA

${ }^{26}$ Department of Astronomy and Astrophysics and the Fermi Institute, The University of Chicago, Chicago, IL 60637, USA Received 2012 August 10; accepted 2013 May 15; published 2013 July 12

\begin{abstract}
We present the design and performance of the multi-object fiber spectrographs for the Sloan Digital Sky Survey (SDSS) and their upgrade for the Baryon Oscillation Spectroscopic Survey (BOSS). Originally commissioned in Fall 1999 on the $2.5 \mathrm{~m}$ aperture Sloan Telescope at Apache Point Observatory, the spectrographs produced more than 1.5 million spectra for the SDSS and SDSS-II surveys, enabling a wide variety of Galactic and extra-galactic science including the first observation of baryon acoustic oscillations in 2005. The spectrographs were upgraded in 2009 and are currently in use for BOSS, the flagship survey of the third-generation SDSS-III project. BOSS will measure redshifts of 1.35 million massive galaxies to redshift 0.7 and Ly $\alpha$ absorption of 160,000 high redshift quasars over $10,000 \mathrm{deg}^{2}$ of sky, making percent level measurements of the absolute cosmic distance scale of the universe and placing tight constraints on the equation of state of dark energy. The twin multi-object fiber spectrographs utilize a simple optical layout with reflective collimators, gratings, all-refractive cameras, and state-of-the-art CCD detectors to produce hundreds of spectra simultaneously in two channels over a bandpass covering the near-ultraviolet to the near-infrared, with a resolving power $R=\lambda / \mathrm{FWHM} \sim 2000$. Building on proven heritage, the spectrographs were upgraded for BOSS with volume-phase holographic gratings and modern CCD detectors, improving the peak throughput by nearly a factor of two, extending the bandpass to cover $360 \mathrm{~nm}<\lambda<1000 \mathrm{~nm}$, and increasing the number of fibers from 640 to 1000 per exposure. In this paper we describe the original SDSS spectrograph design and the upgrades implemented for BOSS, and document the predicted and measured performances.
\end{abstract}

Key words: cosmology: observations - instrumentation: spectrographs - surveys

Online-only material: color figures 


\section{INTRODUCTION}

The Sloan Digital Sky Survey (SDSS; York et al. 2000) project was conceived in the mid-1980s as an ambitious endeavor to understand the large-scale structure of the universe. SDSS and its extension, SDSS-II, conducted a coordinated imaging and spectroscopic survey from 2000-2008 over approximately $10,000 \mathrm{deg}^{2}$ of high Galactic latitude sky. Now in its third phase of operation, SDSS is one of the most successful projects in the history of astronomy. The survey has produced an enormous catalog consisting of five-band digital images that include nearly one billion unique objects, and spectra of 930,000 galaxies, 120,000 quasars, and 460,000 stars, all publicly available (Abazajian et al. 2009, and references therein).

To obtain these imaging and spectroscopic data, a dedicated $2.5 \mathrm{~m}$ telescope (Gunn et al. 2006), wide-field mosaic CCD camera (Gunn et al. 1998), and twin multi-object fiber spectrographs were constructed and installed at the Apache Point Observatory (APO) in Sunspot, New Mexico. The telescope, built to accommodate the requirements for both imaging and spectroscopy, is shared by the camera and spectrographs, which mount at the Cassegrain focus. The imaging survey was carried out on clear, dark nights with good seeing using the 120 mega-pixel camera, which operated in drift-scanning mode using a $5 \times 6$ array of $2048 \times 2048$ pixel detectors to obtain ugriz (Fukugita et al. 1996), photometry. The imaging data, once reduced and calibrated (Smith et al. 2002; Pier et al. 2003; Ivezić et al. 2004; Tucker et al. 2006; Padmanabhan et al. 2008), were used for spectroscopic target selection. Spectroscopy was performed using the two multi-object fiber spectrographs, collecting 640 spectra over the $3^{\circ}$ diameter field in one exposure.

In this paper, we describe the design and performance of the SDSS spectrographs, and their recent upgrade for the Baryon Oscillation Spectroscopic Survey (BOSS; Schlegel et al. 2009; Dawson et al. 2013). BOSS is the flagship survey in the thirdgeneration SDSS-III program currently underway at the $2.5 \mathrm{~m}$ SDSS telescope (Eisenstein et al. 2011). BOSS will measure the cosmic expansion history of the universe to percent-level precision by mapping an immense volume of sky to obtain the spatial distributions of galaxies and quasars, and from it, the characteristic scale imprinted by baryon acoustic oscillations (BAO) in the early universe (for a review of BAO with a respect to other cosmological probes, see Weinberg et al. 2013). A measure of the scale at low redshifts, out to $z \sim 0.7$, will be obtained by carrying out a redshift survey of 1.35 million massive galaxies from 10,000 $\mathrm{deg}^{2}$ of SDSS data. BOSS will also observe Ly $\alpha$ absorption in the spectra of 160,000 highredshift quasars to measure large-scale structure at redshifts of $z \sim 2.5$.

Each SDSS spectrograph utilizes a dual-channel design with a common reflecting collimator and a dichroic to split the beam into a blue channel and a red channel. In each channel, just downstream of the dichroic, a transmitting grism disperses the light, which is imaged by an all-refractive camera onto a CCD. For BOSS, the basic optical design has been retained, with several improvements. The ruled gratings have been replaced by volume-phase holographic (VPH) grisms (gratings sandwiched between two prisms) and the CCDs have been replaced with more modern devices. These changes produce a significant improvement in throughput and a modest extension of the wavelength range in both the blue and red channels. Additionally, smaller diameter fibers that are better matched to the angular scale of BOSS targets have been installed, allowing the total number of simultaneous spectra obtained from the two spectrographs to be increased from 640 in the original design to 1000 in the BOSS configuration.

The remainder of this paper is organized as follows. In Section 2 we begin by describing the design and construction of the original SDSS spectrographs in some detail, published here for the first time. This is followed in Section 3 by a discussion of the spectrograph upgrades completed in 2009 for BOSS. The performance of both the original SDSS spectrographs and the upgraded BOSS design is presented in Section 4. Finally, some highlights of the scientific research enabled by these instruments is provided in Section 5.

\section{SDSS SPECTROGRAPH DESIGN}

\subsection{Design Requirements}

The requirements for the SDSS spectrographs were set by its primary scientific goal: the creation of a three-dimensional widearea map of the universe to reveal its large-scale structure. The SDSS imaging survey provides the two-dimensional locations of nearly one billion celestial objects, and spectroscopy of a selected subset of targets is then used to determine redshifts and thus distances. The project set as a requirement spectroscopy of one million galaxies and 100,000 quasars distributed over approximately $10,000 \mathrm{deg}^{2}$.

Acquisition of a large number of spectra simultaneously over a large field of view, with moderate resolution sufficient for accurate redshift measurements, naturally led to the choice of a fiber-fed multi-object spectrograph. The spectrograph design was dictated in large part by the design of the telescope, which was itself optimized for both wide-field, multi-band, imaging and multi-object spectroscopy. Requirements were specified when possible; however, the instrument design was largely driven by technology available at the time.

In what follows throughout Section 2.1, we summarize the requirements that dictated the design of the SDSS spectrographs.

\subsubsection{Telescope Design}

The $2.5 \mathrm{~m}$ SDSS telescope is a modified distortion-free Ritchey-Chrétien design with a $3^{\circ}$ diameter field of view, and $f / 5$ final focal ratio, which provides a good match to fibers for spectroscopy $\left(180 \mu \mathrm{m}\right.$ diameter, $\left.3^{\prime \prime}\right)$ and to the imaging CCDs (pixel size $24 \mu \mathrm{m}, 0$, 4 ). The optical design incorporates two aspheric corrector lenses, a Gascoigne-type design located near the vertex of the primary mirror, and two interchangeable secondary correctors, one used for imaging and the other for spectroscopy. The imaging corrector is a thick fused silica lens located close to the focal plane and is incorporated into the SDSS camera, where it serves a mechanical function in addition to providing optical correction. The spectroscopic corrector is a thinner lens located further from the focal plane and optimized for chromatic focus. The plate scale for spectroscopy is $3.627 \mathrm{~mm} \operatorname{arcmin}^{-1}$. The spectroscopic focal surface is slightly curved, with a maximum deviation from a plane of $2.6 \mathrm{~mm}$. One important detail of the spectroscopic optics is that the central ray for each field point is not perpendicular to the focal plane, necessitating a clever correction scheme for fiber placement that will be described below.

\subsubsection{Number of Fibers}

Spectroscopy of approximately one million objects over $10,000 \mathrm{deg}^{2}$, plus $10 \%-20 \%$ additional fibers for calibration 
sources and sky background measurements, implies a density of $120 \mathrm{deg}^{-2}$. The $2.5 \mathrm{~m}$ telescope has a field of view of $7 \mathrm{deg}^{2}$, but each plate will view a unique area on the sky of about $5 \mathrm{deg}^{2}$. The higher density of plates is due to the need for overlap between fields to ensure complete sky coverage without gaps, and to allow multiple observations to cross-calibrate the entire survey. The required number of fibers is therefore approximately 600 per plate.

A practical limit on the number of fibers was imposed by the detector format, camera design, and fiber mounting scheme. For proper spectral sampling, the fiber images on the detector should be about 3 pixels in diameter, with an equal space between spectra to reduce crosstalk and allow for a measurement of the scattered light floor. Thus, each spectrum used six detector columns, and the $2048 \times 2048$ pixel detector could accommodate a maximum of 341 spectra. The actual number was reduced to 320 spectra to avoid camera optical distortions near the detector edges and to allow for extra gaps between groups of 20 fibers, which was necessary for the fiber mounting scheme described in Section 2.2. These larger gaps turned out to be quite useful for measurements of scattered light in the wings. The final choice of 640 fibers per plate, or 320 per spectrograph, provided some contingency over the required 600 fibers, allowing for broken fibers, additional calibration fibers, and/or ancillary programs utilizing the extra fibers.

\subsubsection{Fiber Diameter}

The fiber diameter is set by the desire to maximize the signal-to-noise ratio $(\mathrm{S} / \mathrm{N})$ for an extended source given the sky background. For the galaxies of interest around redshift $z=0.1$ and the sky conditions at Apache Point, this corresponds to a fiber size of around $3^{\prime \prime}$, or a fiber diameter of $180 \mu \mathrm{m}$. Fibers of good optical quality were also readily obtainable in this size.

\subsubsection{Wavelength Range}

Redshifts are determined either from absorption lines or emission lines - in both cases only a few lines contribute most of the signal. In absorption, three features are dominant: the $\mathrm{Mg}$ b triplet at $\lambda=5180 \AA, \mathrm{Ca}$ at $\lambda=5270 \AA$, and the Na I doublet (D lines) at $\lambda=5890 \AA$. At shorter wavelengths, the Ca II K and $\mathrm{H}$ lines at $\lambda=3933,3969 \AA$ and the $G$ band $4300 \AA$ may also be detected in absorption. In emission, $\mathrm{H} \alpha=6353 \AA$ is the strongest (and often the only) line, although the [O II] doublet may also be visible at $\lambda=3727 \AA$.

Given the availability of these spectral features, and considering practical limitations on UV throughput, the short wavelength cutoff was set at $3900 \AA$ to ensure that the $\mathrm{H}$ and $\mathrm{K}$ lines of Ca II are observable even at zero redshift, while the [O II] doublet is observable at $z>0.05$. Redshift determination for most nearby galaxies could have been accomplished with a single blue arm extending up to $6000 \AA$; however, the SDSS imaging camera was designed to measure to the detector red limit cutoff, so it was decided to take advantage of the detector sensitivity in the spectrographs and add the red channel. This would enable observation of $\mathrm{H} \alpha$ to a redshift of $z=0.2$ or more, as well as the observation of quasars out to redshifts beyond $z=5$.

Extension of the upper wavelength cutoff to $9100 \AA$ opened up a rich new vein of scientific discovery that was not anticipated at the time of the instrument design. In particular, pushing the long wavelength cutoff as high as possible extended the limit for redshift determination of luminous red galaxies (LRGs) using the $4000 \AA$ break. The LRG sample (Eisenstein et al. 2001) was used to make the first observation of the BAO feature, which in turn motivated the future upgrade of the spectrographs to even longer wavelengths for BOSS, as discussed later in this paper.

\subsubsection{Resolving Power}

The spectroscopic resolution is defined as the full width at half-maximum (FWHM) of the one-dimensional point-spread function (PSF), in wavelength units (a resolution element). The resolving power is the wavelength divided by this quantity, and we will often use the phrase "higher resolution" to mean higher resolving power, as is the normal usage. Given a fixed number of pixels in the dispersion direction and requiring proper sampling, increasing resolving power reduces the wavelength range. Higher resolving power also reduces the number of source photons per pixel, increasing the exposure time required to exceed the CCD read noise. On the other hand, if the resolving power is too low, absorption lines cannot be resolved and this will ultimately degrade both the accuracy and success rate of redshift measurements.

The resolution was therefore set by the requirement to obtain spectroscopic redshifts of galaxies to an accuracy limited only by the broadening due to typical velocity dispersions of 100 to $200 \mathrm{~km} \mathrm{~s}^{-1}$. This corresponds to a resolving power of 1500-3000.

The actual resolving power as a function of wavelength was allowed to vary within these limits to optimize the red-blue channel wavelength split location and the total wavelength coverage, while maintaining well-sampled spectra with 3 pixels per resolution element on the $\mathrm{CCD}$ over the full wavelength range. These choices of spectrograph parameters were chosen to optimize the overall redshift success rate for a given exposure time.

\subsubsection{Throughput and Signal-to-noise Ratio}

The requirement on throughput was set by the desire to obtain one million spectra over $10,000 \mathrm{deg}^{2}$ to a limiting Petrosian magnitude of $r=18.15$ in five years, corresponding to roughly $100 \mathrm{deg}^{-2}$ galaxies. Given the number of fibers and average weather at APO, this implied an average exposure time of one hour.

Provided that the spectral resolution is sufficient to resolve the absorption lines, the minimum $\mathrm{S} / \mathrm{N}$ needed to derive a redshift depends mainly on the strength of the absorption lines. For convenience, the $\mathrm{S} / \mathrm{N}$ per $\AA$ of the spectral continuum will be quoted. For an elliptical galaxy with strong absorption features, spectra obtained in the Center for Astrophysics redshift surveys (Huchra et al. 1983; Falco et al. 1999) demonstrated that one can measure a reliable redshift with $\mathrm{S} / \mathrm{N}$ per $\AA>8$, i.e., one needs to collect 64 object photons per $\AA$, assuming that the noise is dominated by photon statistics from the source. This number must be increased, however, if sky background and/or readout noise is significant. A significant problem for some galaxies is that they have weak absorption lines (presumably because they have a significant amount of light from earlytype stars) and yet lack strong $\mathrm{H} \alpha$ emission. In these cases one may need two or three times as many photons to derive an absorption-line redshift. We adopt as a guide the goal of obtaining spectra with $\mathrm{S} / \mathrm{N}$ of 15 per $\AA$. Simulated galaxy and quasar spectra indicated that we could in fact reach this goal with exposures of somewhat less than one hour in typical conditions for seeing and atmospheric extinction. The corresponding throughput requirement, including atmospheric extinction and the telescope throughput, varies as a function of wavelength; the maximum requirement is about $17 \%$ at $7000 \AA$, 


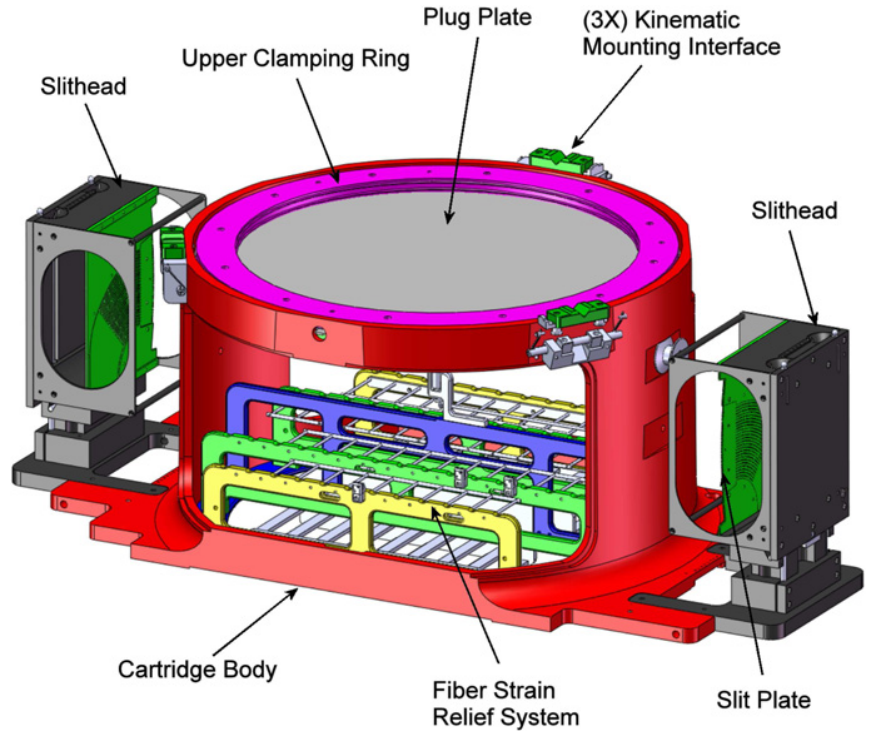

Figure 1. Rendering of a fiber cartridge. The fiber cartridge consists of a cast aluminum body that supports the fiber harness, the two slitheads, and the plugplate, which has a diameter of $800 \mathrm{~mm}$. The slitheads are attached to the cartridge body with a spring-loaded seating system that provides alignment for insertion into the spectrograph bodies, but then allows the slithead to float free from the cartridge body and engage the slithead-to-spectrograph kinematic mounting system. Kinematic mounts around the periphery of the cartridge casting ensure accurate and repeatable placement of the cartridge with respect to the telescope.

with requirements of roughly $10 \%, 15 \%$, and $10 \%$ at $4000 \AA$, $6000 \AA$, and $8000 \AA$, respectively.

\subsection{Fiber System Design}

\subsubsection{Overview of Fiber System}

Light is transmitted from the telescope focal plane to two identical spectrographs by fiber optic strands $180 \mu \mathrm{m}$ in diameter ( $3^{\prime \prime}$ on the sky). Light enters the fibers at the telescope focal plane in a cone of numerical aperture $0.1(f / 5$ beam), and the spectrograph collects light emitted from the other end of the fibers in a slightly larger cone with numerical aperture 0.125 $(f / 4)$, due to focal ratio degradation (FRD) that occurs as the light travels down the fiber. Any light emitted outside this cone will be lost, so a primary requirement on the fiber system is to limit FRD so as to maximize throughput. To this end, the spectrographs are mounted on the telescope to avoid any relative motion between the two ends of the fibers and the potential stress that can result in increased FRD (an issue that was not well understood at the time). References available in those days (early 1990s) suggested that the macrobending of fibers is benign (Angel et al. 1977; Heacox 1986; Clayton 1989); however, a recent study shows that the repeated bending of fibers, as would be the case for a bench-mounted spectrograph, can increase FRD over several years of operation (Murphy et al. 2012). This scheme also maximizes throughput by keeping the fibers short, minimizes fiber throughput variations due to physical motion and stress, and avoids the problems of routing and protecting long fiber runs. The sky ends of the fibers are plugged into drilled $800 \mathrm{~mm}$ diameter aluminum plates called plug-plates that position the fibers on the spectrograph focal plane, and the other ends of the fibers are terminated in one of two slitplates. Each thin slitplate is mounted to a rigid frame with precision locating features for accurate placement in the spectrograph. The assembly of plug-plate, fibers, and slitheads is mechanically supported by a portable aluminum cartridge that can be

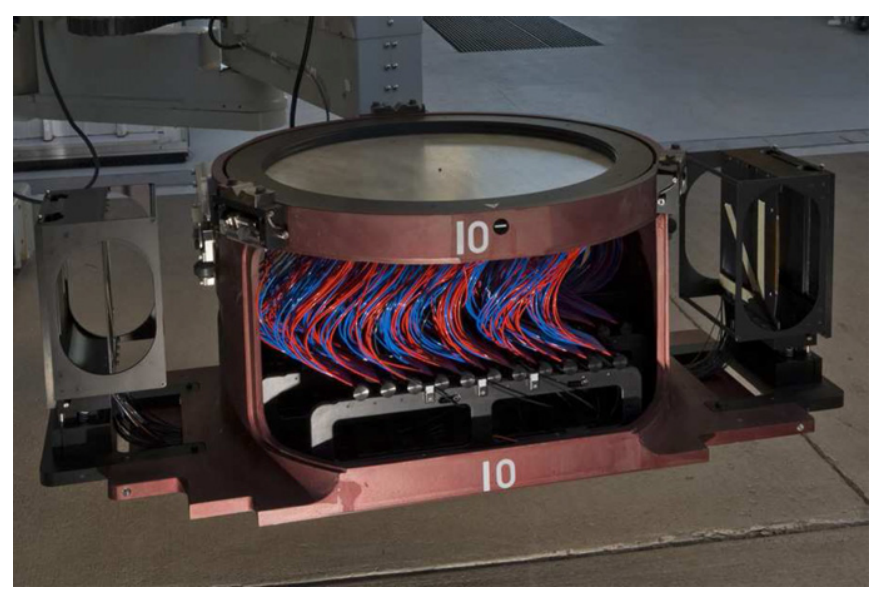

Figure 2. Photograph of a BOSS fiber cartridge. Fibers plugged into the back of the plug-plate are routed in bundles to the slitheads (the two boxes standing upright at the left and right side of the cartridge). The design shown is identical to that used for SDSS except for the number and size of the fibers. For SDSS, 320 fibers are routed to each slithead, while for BOSS each slithead carries 500 fibers.

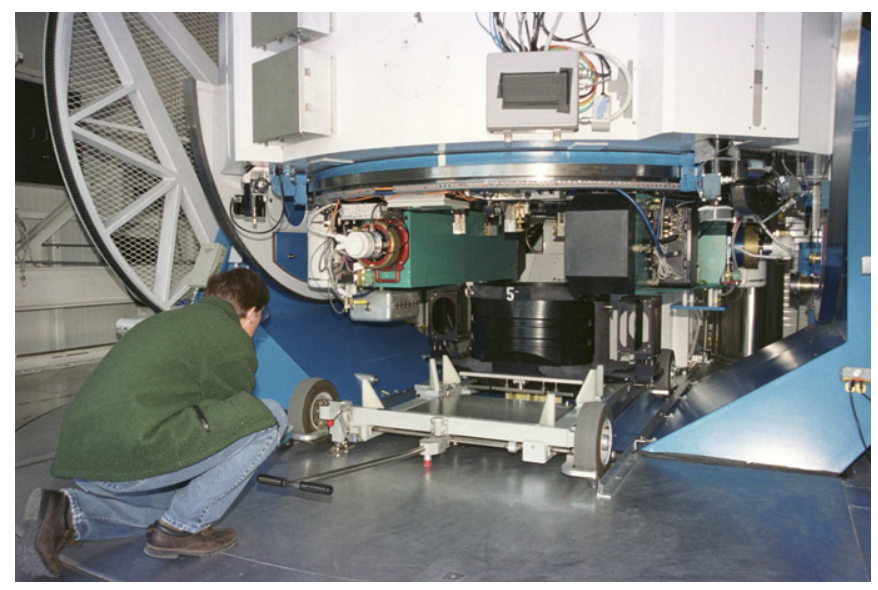

Figure 3. Photograph showing a fiber cartridge being installed on the telescope. The twin spectrographs are the green instruments on either side of the focal plane. The cartridge is raised by a hydraulic lift in the floor below the primary cell. When raised, the cartridge engages kinematic mounts for precise location. At the same time, the two slitheads engage the spectrographs, each of which is located by its own kinematic mounting features integral to the slithead and spectrograph optical bench. Installation takes approximately three to five minutes.

installed on the telescope by a single operator in a few minutes. New plug-plates are mounted on the cartridges during the day and plugged with fibers, then sequentially mounted on the telescope during the night. A rendering and photograph of a fiber cartridge are shown in Figures 1 and 2, respectively.

For each new sky field, a cartridge is wheeled under the telescope using the Linde cart (named for its designer Carl Lindenmeyer) and attached to the telescope rotator using pneumatic clamps. At the same time the attached slitheads enter the spectrographs through the open slithead doors and are clamped in place. A kinematic mounting interface ensures accurate, repeatable placement. The photograph in Figure 3 illustrates the operation. Eight cartridges were fabricated for SDSS to provide sufficient pre-plugged plates for an entire night of observing.

Each cartridge also has a set of coherent fibers that are placed on pre-selected guide stars and viewed by the guider camera. These stars are used for field rotation and translation to align the plug-plate to the field. 


\subsubsection{Cartridges}

The fiber cartridge consists of a machined aluminum cast body that supports the optical fiber harnesses, spectrograph slitheads, and plug-plate. Assembling these components into a single robust unit protects the fragile fibers during the manipulations necessary for plugging, transport to and from the telescope, and mounting onto the instrument rotator. The cartridges are plugged during the day, and are designed so they can be quickly installed on the telescope at night under often difficult conditions of low light and cold temperatures.

The plug-plate holder consists of two large bending rings that warp the plug-plate to match the telescope best-focus surface. An adjustment rod centered on the back side of the plug-plate is used to fine-tune the plate curvature. The bending rings are mounted to the cartridge body with a set of kinematic pin mounts. The alignment of the cartridge to the telescope is provided by another kinematic mount employing v-groove blocks on the cartridge that engage with v-blocks on the telescope. This system ensures proper and repeatable alignment between the plug-plate and telescope focal plane.

The two slitplates, each supporting 320 fibers, are mounted in their respective slitheads. These slitheads are aluminum assemblies mounted outboard of the cartridge body that support and protect the slitplates. The slitheads are attached to the cartridge body with a spring-loaded seating system that provides alignment for insertion into the spectrograph bodies, but then allows the slithead to float free from the cartridge body and engage the slithead-to-spectrograph kinematic mounting system. When not mounted on the telescope, these slitheads are protected by sliding covers to prevent contamination and/or mechanical contact with the delicate slitplates.

All cartridge operations occur at the same elevation, on the telescope platform and the adjacent support building. In the plugging lab, the exposed plug-plates from the previous night's observing are unplugged and removed from their cartridges and new plates are installed. Once plugging and fiber mapping is completed (a process that takes $30-45$ minutes), the $145 \mathrm{~kg}$ cartridge is stowed on a lift table installed in a bay that provides both interior and exterior bay door access. At night, the outside door is opened to allow the cartridges to equilibrate to the temperature of the ambient air.

To install a new cartridge on the telescope, an outside manipulator arm is employed to move the cartridge from the storage bay to one of two receiving plates on the Linde cart. The cartridge is then wheeled from the storage bay to the telescope. With the telescope parked at zenith and locked into position, the Linde cart is rolled under the mounted cartridge to align the empty receiver plate with it. Aided by a hydraulic lift, the observer removes the exposed cartridge from the telescope. Then, maneuvering the Linde cart to align the unexposed cartridge onto the hydraulic lift, the observer mounts the new cartridge onto the telescope instrument rotator. Once the new cartridge is latched and the cart receiver plate is lowered back onto the Linde cart, the cart is rolled out from under the telescope. The telescope is now ready to move to the next field and to begin another exposure. Only three to five minutes is required to perform this cartridge change.

As the cartridge is lifted into place and clamped to the telescope, the slitheads are simultaneously inserted into sockets in the spectrographs. The slitheads are attached to the cartridge frame by stiff springs so that they can move slightly with respect to the rest of the cartridge. Once the cartridge has been correctly positioned and clamped to the telescope, each slithead is loaded

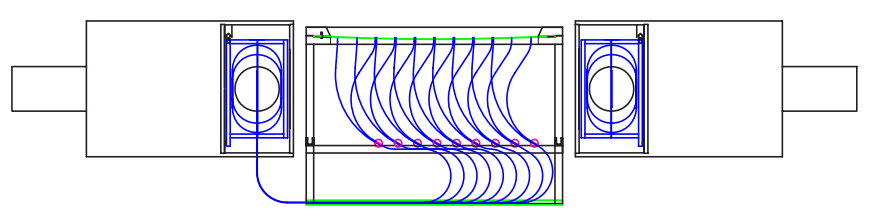

Side View

(only 9 fiber harnesses shown)

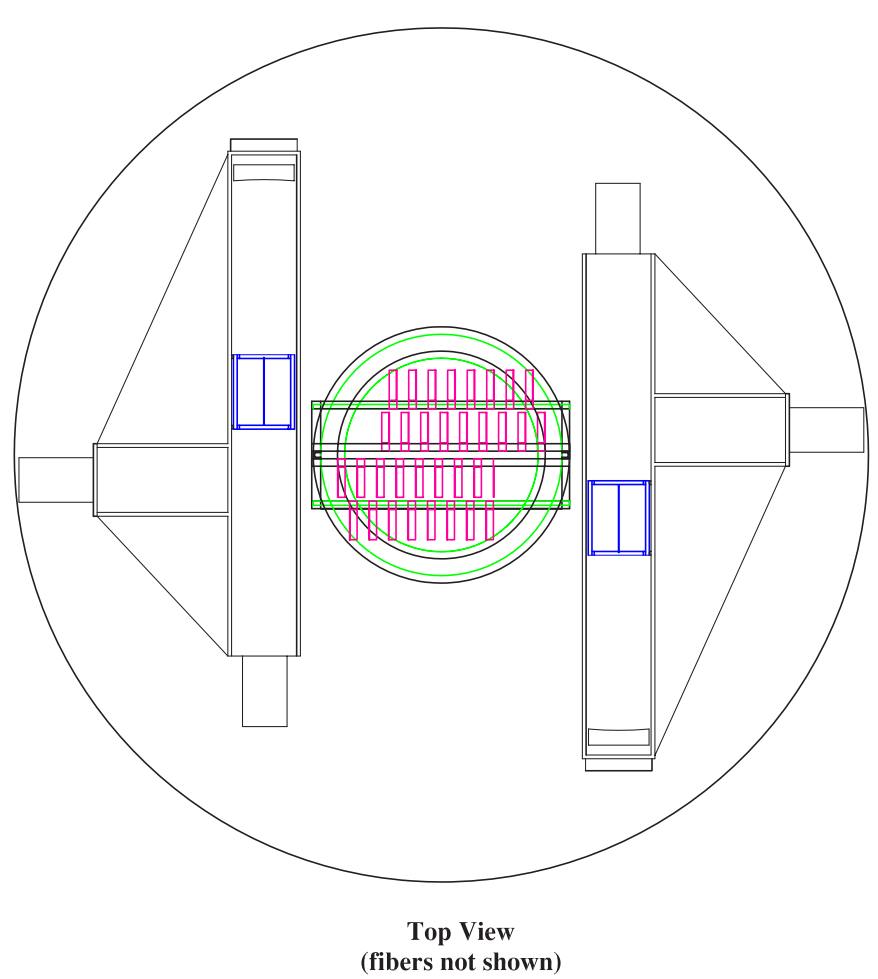

Figure 4. Two schematic views of the cartridge mounted on the telescope. Top: a cutaway side view showing the slitheads inserted into the spectrographs (only nine fiber harnesses are shown). Bottom: bottom view showing the cartridge located between the two spectrographs, which are mounted to the instrument rotator (depicted as the large outer circle).

(A color version of this figure is available in the online journal.)

against a three-point kinematic mount on the spectrograph by a single pneumatic clamp. A flexible rubber seal between the slitheads and the spectrograph bodies prevents extraneous light from entering during exposures. Each slithead is coded and its identification relayed to the observer's workstation when it is inserted. This information allows adjustments for each slithead, e.g., image placement on the CCD and focus, to be made automatically. Figure 4 shows two schematic views of the cartridge mounted on the telescope with the slitheads inserted into the spectrographs.

\subsubsection{Optical Fiber}

The selected optical fiber material is a silica UV-enhanced step-index fiber with a core diameter of $180 \mu \mathrm{m}$, a thin cladding and a polyimide protective layer. The actual fiber was Polymicro Technologies, Inc. ${ }^{27}$ FHP 180-198-218, where the numbers refer to the diameter of the bare fiber, plus cladding and plus polyimide buffer.

\subsubsection{Fiber Harnesses}

A fiber harness consists of 20 fibers of length $1.865 \pm 0.025 \mathrm{~m}$; each fiber cartridge contains 32 fiber harnesses. The fiber

\footnotetext{
27 Polymicro Technologies, Inc., http://www.polymicro.com
} 


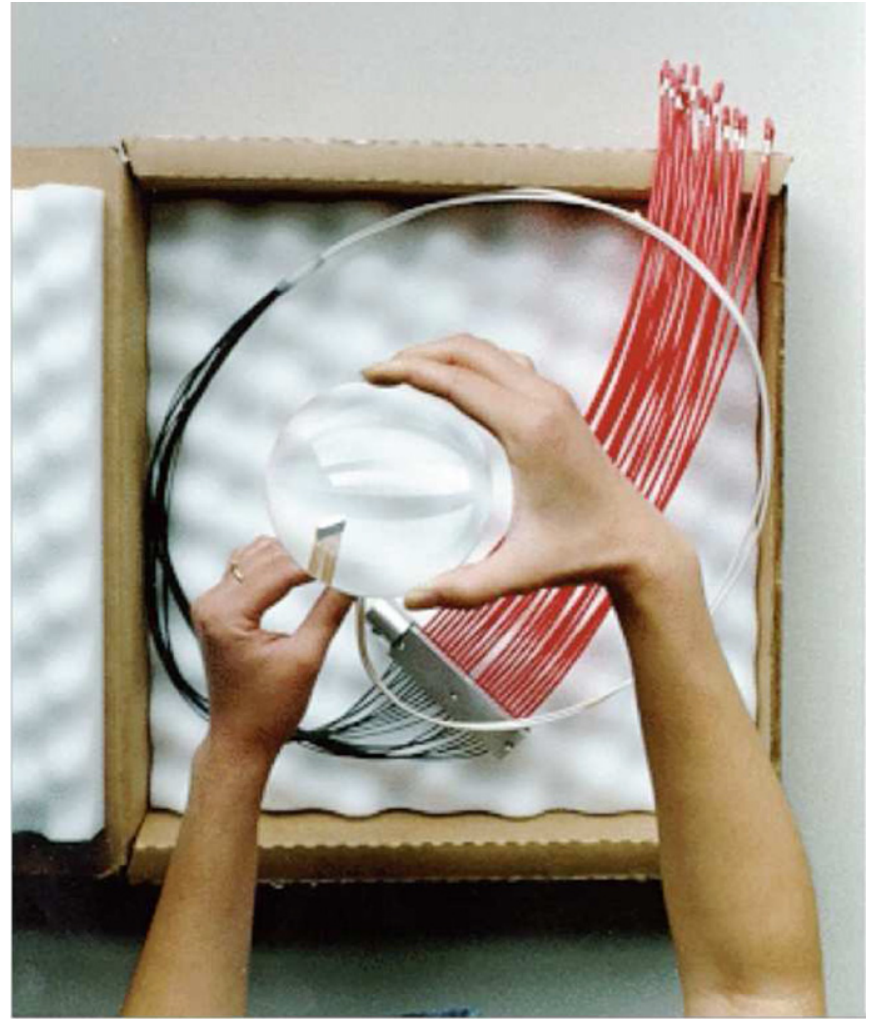

Figure 5. Photograph of an SDSS fiber harness. The ends, which are plugged and unplugged during operations, are protected by tough nylon tubing, which terminates in the anchor block. The lens enlarges the view of the v-groove block termination at the slit end.

harness is the unit procured from C Technologies, Inc. ${ }^{28}$ the vendor responsible for preparing the fibers by terminating both ends. A fiber harness is shown in the photograph in Figure 5.

The plugging end is terminated by gluing it into a ferrule and polishing the end. The ferrule is machined from stainless steel; the most important tolerances on the ferrule are the centering and angle of the hole in which the fiber is inserted, specified at $12 \mu \mathrm{m}$ and 1.1 , respectively. The tilt of the plane of the polish on the fiber end is specified at less than 1.0. The fiber is glued into the ferrule using a low-shrinkage adhesive (MasterBond $\mathrm{EP} 21 \mathrm{AR}^{29}$ ) selected to retain flexibility for many years over a temperature range $-20^{\circ} \mathrm{C}$ to $+30^{\circ} \mathrm{C}$. To facilitate handling the fiber assemblies during plugging and unplugging while protecting the fragile fibers, a protective jacket $380 \mathrm{~mm}$ long is attached to the ferrule on one end and glued into an anchor block on the other end. The selected jacket material consists of nylon tubing $3.2 \mathrm{~mm}$ in diameter, allowing the fiber to move freely inside to avoid imparting any stress. The anchor block holds 20 fiber jackets in a row on $5.1 \mathrm{~mm}$ centers. The anchor blocks are attached to the cartridge body, providing a fixed point from which the fibers are routed to the slitplate. The fibers are stationary from the anchor block to the slitplate, and the only portion of the fiber which moves during plugging is protected inside the nylon jacket. Thin tubing, $950 \mathrm{~mm}$ long and $2 \mathrm{~mm}$ in diameter, is used to bundle the fibers into groups of 10 immediately before their termination at the slitplate.

At the slitplate end, 20 fibers at a time are mass-terminated by gluing them (using MasterBond EP21AR) into a machined piece

\footnotetext{
28 C Technologies, Inc., http://www.ctechnologiesinc.com

29 Master Bond, Inc., http://www.masterbond.com
}
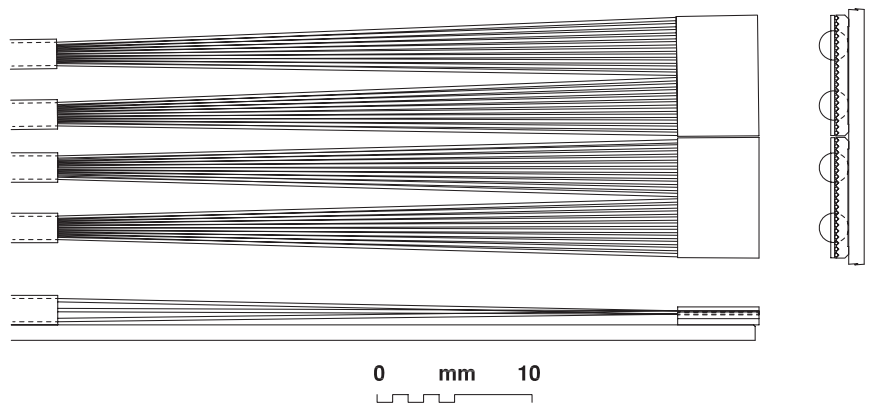

Figure 6. Three views of the v-groove blocks as mounted on the slit plate. Each block contains 20 fibers, which are aligned by $20 \mathrm{v}$-shaped grooves. The fibers are held in place by epoxy sandwiched between the fibers and a cover plate. A hole in the slit plate under each block permits easy removal for replacement.

of stainless steel called a v-groove block; see Figure 6. This item is so named because it has $20 \mathrm{v}$-shaped grooves machined on an electrical discharge machine (EDM) to precisely locate the fibers. The grooves are not parallel, but fan out slightly so they are normal to the tangent of a circle with radius $640 \mathrm{~mm}$. For termination, 20 fibers are arranged side by side in the v-groove block on $390 \mu \mathrm{m}$ centers and glued into place with a cover plate on top. The tolerance on the fiber placement is $30 \mu \mathrm{m}$ (individual, not cumulative), and 0.3 in tilt between the groove and length of the fiber; there is an additional tolerance of 0.3 on the alignment of the grooves with respect to the body of the v-groove block. After the fibers are aligned in the v-groove block, covered and glued in place, the ends are polished on a flat surface, with a specified tolerance of 1.0 on the tilt of the plane of polish.

\subsubsection{Slitplates}

Each spectrograph accepts light from 320 fibers, terminated in $16 \mathrm{v}$-groove blocks that are glued to a thin, rigid aluminum slitplate with a $640 \mathrm{~mm}$ radius of curvature. The center-tocenter spacing between fibers in adjacent v-groove blocks is $523 \mu \mathrm{m}$, compared with $390 \mu \mathrm{m}$ between fibers within a $\mathrm{v}$-groove block. The v-groove block is aligned by locating the output edge against a curved alignment jig; it is fastened to the slit plate with adhesive. The adhesive is metered using an air-powered fluid dispenser to prevent contamination of critical surfaces. The slitplates are in the beam and therefore must be as thin as possible; the total thickness of the plate and v-groove blocks is specified to be less than $3.2 \mathrm{~mm}$.

\subsubsection{Plug-plates}

The telescope optical system is a simple, fast, large field design with a focal surface flat to $2.6 \mathrm{~mm}$ but one where the principal ray deviates from the normal to the best-focus surface by up to $37 \mathrm{mrad}(2.12)$. For highest efficiency, the ends of the optical fibers should be positioned on the best-focus surface with their axes aligned with the principal ray. Plug-plate technology can be made to satisfy these criteria quite nicely.

The plug-plates are an aluminum alloy 2024-T3, $3.2 \mathrm{~mm}$ thick and $800 \mathrm{~mm}$ in diameter. By applying bending moments to the edge of the plate (beyond the field of view), finite element calculations show they can be deformed to match the best-focus surface to an area-weighted $62 \mu \mathrm{m} \mathrm{rms}$. The greatest departure from the best-focus surface is $200 \mu \mathrm{m}$ and occurs at the center where the images are the best. Overall, the images are not significantly degraded from the best-focus surface. 
As deformed to match the best-focus surface, the hole axes should align with the principal ray axes. This configuration is straightforward to accomplish if the plug-plate is deformed (in the opposite sense) over a properly curved mandrel, for drilling. The drilling is performed using a three-axis Computer Numerically Controlled (CNC) milling machine, as it is not necessary to tilt the drilling head or the plug-plate. Plugplate drilling is performed in the Physics Machine Shop at the University of Washington, Seattle, WA.

Drill test results indicate that holes can be drilled with an accuracy of $9 \mu \mathrm{m} \mathrm{rms}$ in position and $4 \mu \mathrm{m} \mathrm{rms}$ in diameter using short high-precision spade drill bits in a custom-made collet. In the test, four different bits were used to drill 50 holes each. The drilling time was $5.8 \mathrm{~s}_{\text {hole }}{ }^{-1}$. No significant degradation in drilling accuracy was observed for a range of slopes in the work-piece surface from 0 to $70 \mathrm{mrad}(4.01)$.

The plug-plates have a mass of $4.3 \mathrm{~kg}$ and are sufficiently thin so that the bending stresses, forces, and material costs are reasonable. They are thick enough to provide hole depth adequate to constrain the plug angular alignment with the hole and to prevent significant gravity-induced deflections.

Once plugged, profilometry is performed on the plug-plates in the plugging laboratory to verify that the surface conforms to the focal plane within tolerances. This is done using a fixture that mounts five digital linear indicators on a steel beam that straddles the plug-plate, using a kinematic support system to make contact with the aluminum. The five indicators measure the plate displacement from a calibrated zero reference along a radial direction from the center to the edge of the plug-plate. There are four sets of measurements taken, spaced $90^{\circ}$ apart for each plug-plate. The measurements are then recorded in a database for each plugging of each plate. ${ }^{30}$

\subsubsection{Fiber Tester}

Each completed fiber harness is tested by the vendor to ensure that each of the 20 fibers meets a minimum throughput requirement of $85 \%$. The test device, which was supplied to the vendor, uses white light from an intensity-stabilized quartzhalogen lamp, which is imaged onto the fiber under test using a source fiber and a microscope objective, which produces a uniform $f / 5$ converging beam. A microscope eyepiece and pellicle beamsplitter provide a view of the input end of the fiber under test.

Light from the output end of the test fiber is collected by a pair of achromatic doublets focused onto a silicon photodiode. A filter between the doublets limits the source to the visible range where the optics perform well. A calibrated aperture blocks light outside a cone of $f / 4$. A computer-controlled translation stage accurately locates the appropriate fiber of the $\mathrm{v}$-groove block in front of the aperture. This same light collection system is also used to measure light from the microscope objective, in order to make absolute throughput measurements.

An identical instrument was used to verify the manufacturer's measurements for some fraction of the fibers (see footnote 30).

\subsubsection{Fiber Mapper}

With 640 fibers on each plug-plate, it was important not to rely on manual plugging of each fiber into a specific preassigned hole. However, plugging cannot be completely random

\footnotetext{
30 Portions of the description provided in this section have been copied from http://www.astro.princeton.edu/PBOOK/welcome.htm and are reproduced here for completeness.
}

because not every fiber can reach every position on the plate; this constraint was adopted to keep the total fiber length relatively short. The solution was to have hand-marked regions on each plate for specific fiber sets that can reach all of the holes within the delineated area, and to then allow for random plugging within those regions. After plugging is complete, the cartridge is wheeled over to a fiber mapper system, which is used to determine which fiber is in which hole.

The fiber mapper illuminates each fiber sequentially by moving a light along the slithead; the illuminated fiber appears as a bright point against the dark background of the plug-plate. A CCTV camera behind a narrow band filter matching the light source determines the $\mathrm{X}-\mathrm{Y}$ position of each fiber on the plugplate. This $\mathrm{X}-\mathrm{Y}$ position is converted to sky coordinates and stored in the database, providing a map between the physical location of each spectra on the CCD and the corresponding coordinates on the sky. The fiber mapper is also useful for identifying broken or loose fibers that drop down into the hole after plugging. The entire operation takes about five minutes.

\subsection{Optical Design}

\subsubsection{Optical Design Overview}

The optical design of the spectrographs was strongly influenced by a few considerations including, the use of optical fibers to feed light from the telescope to the spectrographs, available large-format science-grade CCDs, the required bandpass, and the required resolving power. The exit face of a fiber, which is the entrance aperture into the spectrograph for one object, must be collimated, dispersed, and finally re-imaged onto a detector with adequate pixel sampling. At the time, Tektronix/SITe $2 \mathrm{k} \times 2 \mathrm{k}$ format CCDs having $24 \mu \mathrm{m}$ pixels were state-of-theart. Given a fiber output focal ratio of $f / 4$, the collimated beam must be re-imaged by a camera with a focal ratio of $f / 1.5$ to provide sufficient demagnification to achieve 3 pixel images of the fibers on the $24 \mu \mathrm{m}$ pixel pitch. At this sampling, approximately 700 resolution elements are available across the detector. Clearly, the full 3900-9100 Å bandpass will not fit within a single detector at the required resolving power of $\sim 2000$. Thus, two channels are needed. Lastly, as a practical matter, it was determined that a collimated beam diameter of $160 \mathrm{~mm}$ would be ideal; a larger diameter would begin to require unreasonably large optics, while a smaller beam would require higher field angles into the cameras and therefore more difficult designs. With this beam diameter, the cameras have a focal length of $240 \mathrm{~mm}$, and reasonable grating designs can provide the necessary angular dispersion.

Figure 7 shows the optical layout of the SDSS spectrographs. Light enters each spectrograph through 320 fibers, which terminate at a curved slithead. The slithead positions the fiber ends on a radius concentric with the spherical collimating mirror, which operates at $f / 4$ and produces a $160 \mathrm{~mm}$ diameter beam. The $45^{\circ}$ dichroic beamsplitter reflects the blue portion of the bandpass $(\lambda<6000 \AA)$ and transmits the red wavelengths $(\lambda>6000 \AA)$. Located immediately behind the beamsplitter in each channel is a grism, consisting of a right angle prism with a transmissive surface-relief grating replicated on the hypotenuse. The dispersed light exits the grisms and enters all-refractive, eight-element, $f / 1.5$ cameras. Each camera contains a single Tektronix/SITe $2 \mathrm{k} \times 2 \mathrm{k}$ CCD with $24 \mu \mathrm{m}$ pixels. The camera demagnification from $f / 4$ to $f / 1.5$ produces fiber images that are just under 3 pixels in diameter. A fiber-center separation of $390 \mu \mathrm{m}$ at the slithead produces 6 pixel center-to-center spectra 


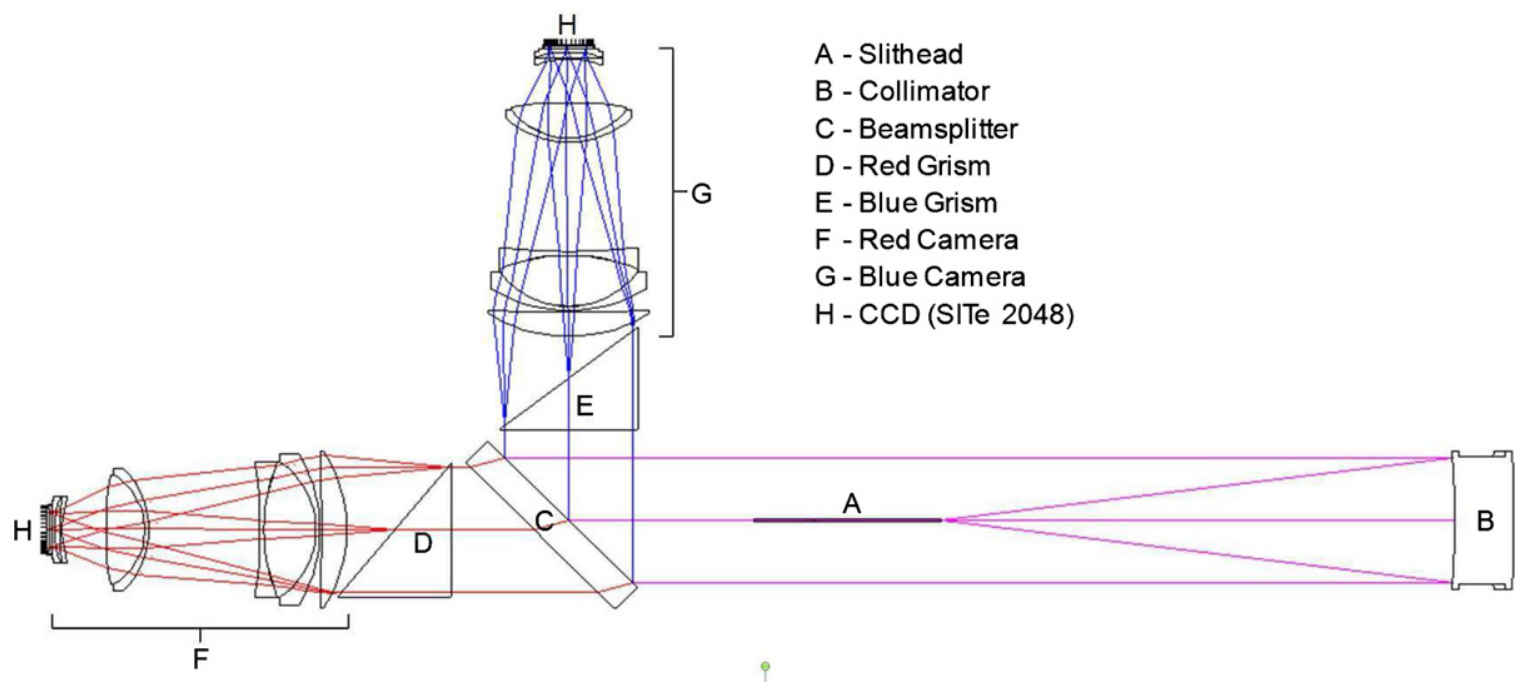

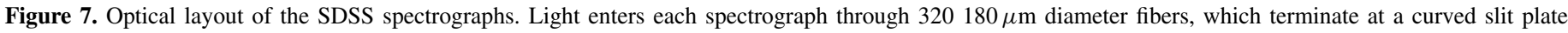

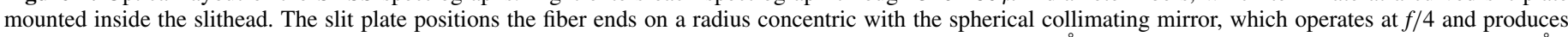

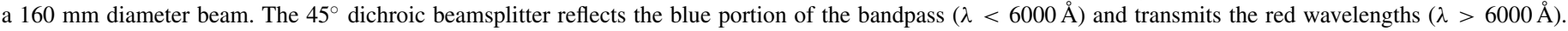

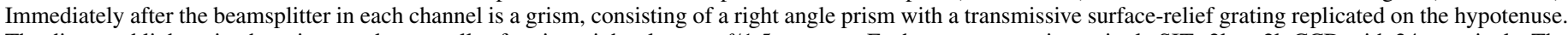

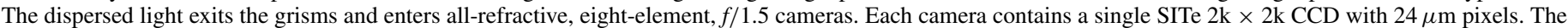
camera demagnification from $f / 4$ to $f / 1.5$ produces fiber images that are just under 3 pixels in diameter, resulting in 3 pixel tall spectra on the detector.

spacing at the detector, sufficient to avoid crosstalk between spectra.

\subsubsection{Slithead}

Light enters the spectrograph through $180 \mu \mathrm{m}$ diameter fibers, which terminate at the curved slit plate; see Section 2.2 for more details on the slithead design. The fibers are stacked vertically to form a tall, narrow slit on a radius whose center of curvature coincides with that of the collimator. Additionally, the fibers are arranged in a fan-like pattern outward from the center of curvature toward the collimator, so that the central ray from each fiber strikes the collimator normal to the surface. Thus, the slit plate is at the focus of a one-dimensional Schmidt collimator.

\subsubsection{Collimator}

In combination with the curved slithead, the spherical collimator mirror forms a corrector-less Schmidt collimator. It was possible to eliminate the corrector plate since some of the reduced performance could be compensated for in the spectrograph cameras; enough to satisfy the imaging requirements. Thus, the final collimator design is a single mirror with a spherical figure. The mirror itself is fabricated from a rectangular Hextek $^{31}$ gas-fusion process borosilicate blank, $175 \mathrm{~mm}$ wide, $419 \mathrm{~mm}$ tall, and $73 \mathrm{~mm}$ thick. The planar blank is slumped by Hextek to near-net radius and then ground and polished to the final radius of $1264 \mathrm{~mm}$. An enhanced silver coating was applied to the optical surface by Denton Vacuum. ${ }^{32}$ The collimator forms a pupil at the center of curvature of the mirror, and this is where the gratings are located in each channel in order to minimize their required size.

Alternative mirror technologies were investigated, including aluminum, eggcrate Zerodur, and monoliths. The gas fusion blank was determined to be optimal given weight and cost considerations. The thermal coefficient of expansion, while

\footnotetext{
31 Hextek Corp., http://www.hextek.com

32 Denton Vacuum LLC, http://www.dentonvacuum.com
}

non-zero and much smaller than aluminum, is an acceptable compromise. Obviously an aluminum mirror would eliminate the need to refocus when the temperature changes, but is expensive and the coatings are tricky. An eggcrate Zerodur mirror is expensive and would require refocusing. Lastly, a monolith would not be much cheaper than the gas fusion mirror, and would be significantly less responsive thermally than the gas fusion mirror.

To compensate for changes in instrument temperature with time, focus adjustment is provided by the collimator mount (see Section 2.4), which enables not only focus adjustment but tip/tilt adjustment as well. A pair of Hartmann doors located in front of the mirror (also described in Section 2.4) allow shifts in the collimator focus to be measured rapidly. Tip/tilt adjustment allows the collimator to be precisely coaligned to the cameras, or at least to an average of the two camera axes. Thus, the center fiber can be positioned at the center of the detector in the spatial direction, and the central wavelength can be positioned at the center of the detector in the spectral direction.

\subsubsection{Beamsplitter}

A dichroic beamsplitter divides the incident collimated beam, reflecting the blue portion of the bandpass $(\lambda<6000 \AA)$ and transmitting red wavelengths $(\lambda>6000 \AA)$. It is fabricated from BK7, is $271 \mathrm{~mm}$ wide, $229 \mathrm{~mm}$ tall, and $38 \mathrm{~mm}$ thick, with the dichroic coating applied to the incident surface. The coating reflects the blue light very efficiently $(R>98 \%)$ and transmits the red light somewhat less efficiently $(T>94 \%$ average, including the reflection loss at the exit surface, which has a high performance broadband antireflection coating). The $10 \% / 90 \%$ zone at the crossover wavelength is approximately $50 \mathrm{~nm}$ wide.

\subsubsection{Gratings}

The dispersing elements are grisms with zero angular deviation at $4960 \AA$ for the blue and $7400 \AA$ for the red. These are right angle $\mathrm{BK} 7$ prisms with a transmission grating replicated on the hypotenuse. While a reflection grating might have 


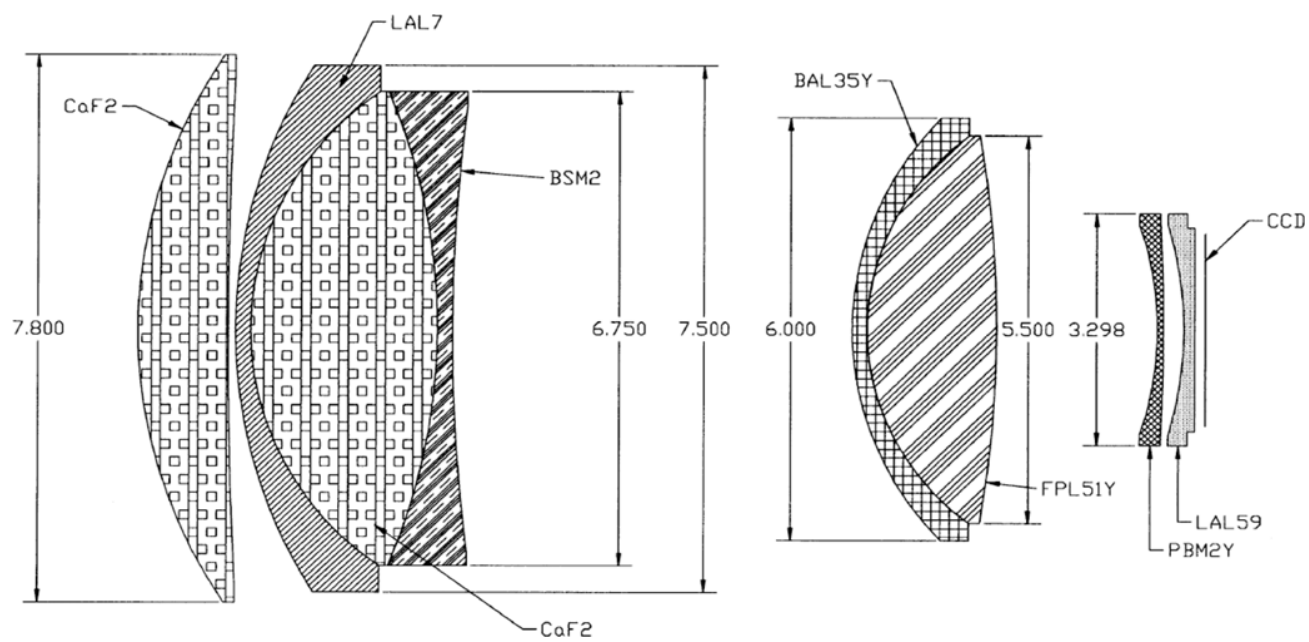

Figure 8. Optical layout of the SDSS spectrograph blue channel camera designed by Harland Epps. Diameters are in inches. The red channel camera layout shares the same eight-element configuration, though the prescription for the doublet and field flatteners differ for the two camera designs. Anti-reflective coatings are optimized for the respective bandpasses of each channel.

been used, the grism permits mounting the cameras close to the system pupil, which is located, approximately, in the middle of the grism. With a reflection grating, the cameras have to be mounted away from the grating to avoid interference with the incoming beam, making them larger and more difficult to design. A plane transmission grating does not work because the diffracted angle is large, making geometric losses high (the groove facets are foreshortened) and forcing the blaze peak outside the optical band. The configuration used here has little groove shadowing or foreshortening and results in high grating efficiency.

The ruling densities are 640 and 440 lines $\mathrm{mm}^{-1}$ for the blue and red grisms, respectively. Because master rulings of the necessary size and groove angle did not exist, new masters were ruled by Hyperfine, Inc.

\subsubsection{Cameras}

The spectrograph cameras are $f / 1.5$ with a $240 \mathrm{~mm}$ focal length and 16.5 deg field of view; see Figure 8 for a layout of the blue camera (the red camera layout is nearly identical). The all-refractive design was chosen to maximize throughput since placing the detector or a secondary mirror in the unobstructed collimated beam, as would be the case for example with a Schmidt camera, would result in significant light loss. Both the red and blue cameras share the same optical prescription for the first four elements (i.e., the singlet and triplet). However, the doublets have unique prescriptions for the red and blue designs, as do the field flatteners. Anti-reflective coatings are optimized for the respective bandpasses of each channel.

Harland Epps (University of California, Santa Cruz) was contracted to design the cameras, using the Low Resolution Imaging Spectrometer (LRIS) spectrograph camera design (Epps 1990) as a starting point with some additional constraints. The most important was to limit aspheric surfaces to one per camera with only mild angles (LRIS had two steep aspheric surfaces). The reason for this was to reduce schedule risk and to increase the vendor pool. The camera employs eight lens elements arranged in five groups, including a contact triplet, a contact doublet, and three singlets. All the surfaces are spherical except for the air side surface of the second element in the doublet, which is a relatively mild asphere. Careful attention was paid to glass selection in order to maximize throughput at $390 \mathrm{~nm}$, with five of the eight elements being either calcium fluoride $\left(\mathrm{CaF}_{2}\right)$ or
Ohara $^{33}$ i-line glasses (which have $>98 \%$ internal transmission at $365 \mathrm{~nm}$ ). Dow Corning Q2-3067 ${ }^{34}$ optical coupling grease was used to join the elements in the doublet and triplet. The resulting design, shown in Figure 8, is similar to LRIS but has a smaller diameter and larger field of view.

Once the fundamental camera design was in hand, it was tuned using Zemax ${ }^{35}$ to match surfaces with existing vendor test plates, optimizing spacings to melt data received from the glass vendor, and generating optical prescriptions for the two field flattening lenses to accommodate the different CCD curvatures. The problem of CCD curvatures was particularly annoying. The CCD surfaces were not flat to start and the shape changed when the devices were cooled to operating temperature. Fortunately, they returned to the same shape every time they were cooled to operating temperature and most had a generally spherical shape that could be corrected with spherical optics. Unfortunately, they were all different so each CCD required a customized pair of field flattening lenses.

\subsubsection{Optical Prescription}

Table 1 shows the spectrograph optical prescription. Some of the surface descriptions can be deciphered from this example: "Doublet,first,back" refers to the doublet component, first lens element, back surface (closest to the CCD). The "Radius" is the radius of curvature in inches, negative implying a concave left surface. "Thickness" is the distance from the current surface to the next; positive to the right. All materials are from Ohara Glass except for $\mathrm{CaF}_{2}$ and the lens couplant, Dow-Corning Q2-3067.

\subsubsection{Predicted Optical Performance}

This section discusses the predicted optical performance of the SDSS spectrographs, including image quality, spectral resolution, and throughput. Measured performance is discussed in Section 4.

Image quality. Spot diagrams for the red and blue channels are shown in Figures 9 and 10, respectively. The spots are shown within a $67.5 \mu \mathrm{m}$ diameter circle, representing the diameter of the imaged fiber on the detector. Each diagram covers the full respective bandpass of the channel, and field points cover the full length of the slit. The average rms spot diameter for the red

\footnotetext{
33 Ohara Corporation, http://www.oharacorp.com

34 Dow Corning, http://www.dowcorning.com

35 Radiant Zemax LLC, http://www.zemax.com
} 
Table 1

Optical Prescription for the SDSS Spectrographs

\begin{tabular}{|c|c|c|c|c|c|}
\hline \multirow[t]{2}{*}{ Surface } & \multicolumn{2}{|c|}{ Radius (mm) } & \multicolumn{2}{|c|}{ Thickness (mm) } & \multirow[t]{2}{*}{ Material } \\
\hline & Blue & Red & Blue & Red & \\
\hline Slithead & -640.080 & -640.080 & 630.123 & 630.123 & Air \\
\hline Collimator mirror & -1263.904 & -1263.904 & -1087.831 & -1087.831 & Air \\
\hline Prism & Plano & Plano & 64.592 & 70.663 & BK7 \\
\hline Grating & $\operatorname{Plano}\left(37^{\circ}\right)$ & Plano(39.5) & 52.578 & 58.420 & Air \\
\hline Singlet,front & -182.804 & -182.804 & -30.531 & -30.531 & $\mathrm{CaF}_{2}$ \\
\hline Singlet,back & -1813.560 & -1813.560 & -2.540 & -2.540 & Air \\
\hline Triplet,first,front & -185.522 & -185.522 & -5.156 & -5.156 & LAL7 \\
\hline Triplet,first,back & -105.867 & -105.867 & -0.076 & -0.076 & Q2-3067 \\
\hline Triplet,second,front & -105.867 & -105.867 & -63.525 & -63.525 & $\mathrm{CaF}_{2}$ \\
\hline Triplet,second,back & 226.136 & 226.136 & -0.076 & -0.076 & Q2-3067 \\
\hline Triplet,third,front & 226.136 & 226.136 & -5.105 & -5.105 & BSM2 \\
\hline Triplet,third,back & -661.416 & -661.416 & -136.931 & -135.941 & Air \\
\hline Doublet,first,front & -110.490 & -109.423 & -5.080 & -5.080 & BAL35Y \\
\hline Doublet,first,back & -87.274 & -81.331 & -0.076 & -0.076 & Q2-3067 \\
\hline Doublet,second,front & -87.274 & -81.331 & -44.120 & -46.990 & FPL51Y \\
\hline Doublet,second,back & $429.006^{\mathrm{a}}$ & $432.892^{b}$ & -53.518 & -53.899 & Air \\
\hline Flattener,first,front & 136.449 & 135.560 & -2.032 & -2.032 & PBM2Y \\
\hline Flattener,first,back & 761.975 & 608.355 & -8.128 & -6.858 & Air \\
\hline Flattener,second, front & 122.733 & 114.427 & -3.810 & -3.810 & LAL59 \\
\hline Flattener,second,back & Plano & Plano & -3.835 & -3.683 & Air \\
\hline $\mathrm{CCD}$ & 2502.992 & -1920.037 & 0 & 0 & Silicon \\
\hline
\end{tabular}

Notes.

${ }^{\text {a }}$ Aspheric surface coefficients: $\alpha_{4}=7.517856 \mathrm{E}-04, \alpha_{6}=-5.900703 \mathrm{E}-05, \alpha_{8}=2.644114 \mathrm{E}-06$.

b Aspheric surface coefficients: $\alpha_{4}=-7.880071 \mathrm{E}-04, \alpha_{6}=6.800388 \mathrm{E}-05, \alpha_{8}=-2.676036 \mathrm{E}-06$.

channel is $21.5 \mu \mathrm{m}$ and the maximum rms diameter is $29.2 \mu \mathrm{m}$. For the blue channel, the average rms diameter is $21.1 \mu \mathrm{m}$ and the maximum rms diameter is $27.3 \mu \mathrm{m}$. When convolved with the $67.5 \mu \mathrm{m}$ imaged fiber diameter, the geometric aberrations represented in these figures degrade the image, but to a minimal degree, and the resulting image width is well sampled by 3 pixels $(72 \mu \mathrm{m})$ on the detector.

Spectral resolving power. To analyze the spectral resolving power, defined as $\lambda / \delta \lambda$, where $\delta \lambda$ is taken to be the spectral FWHM of the fiber image on the detector in $\AA$, the following procedure was used. In Zemax, a circular source with a diameter equal to that of the fiber was placed at the center fiber location on the slit for the wavelength under consideration. Many thousands of rays were launched from this circular source with a uniform distribution, and the resulting image recorded on a simulated detector with pixels $1 / 4$ the size of the $24 \mu \mathrm{m}$ CCD pixels (in order to better sample the image). The simulated image data was analyzed to determine the FWHM, without collapsing the image. The FWHM thus determined was taken to be the resolution, and the resolving power is plotted in Figure 11.

In reality, the resolving power of the instrument is determined using collapsed spectra, so the prediction here is on the low side. However, the Zemax analysis assumes perfect optics and alignment. For this reason, the predictions have been based on uncollapsed spectra, which is the conservative approach. The expectation was that the actual resolving power would be somewhat better than shown here.

Throughput. The total throughput, on-sky, for the SDSS spectrographs was predicted from an end-to-end component model as a function of wavelength. Included in the model were: atmospheric extinction, seeing (slit) losses, telescope, fibers, collimator, dichroic, grism, camera, and CCD. Atmospheric extinction was modeled at one airmass using a Palomar curve ${ }^{36}$ scaled to

\footnotetext{
36 http://www.sdss.org/dr3/instruments/imager/filters/index.html
}

Apache Point using mean values for photometric nights during 1998-2008 (Table 3 of Padmanabhan et al. 2008). Seeing losses were modeled using a double Gaussian PSF with a FWHM of $1^{\prime \prime}$ centered in a $3^{\prime \prime}$ aperture, resulting in a $5.5 \%$ throughput loss assumed flat across the bandpass. The telescope efficiency is based on measured data for $\mathrm{CO}_{2}$-cleaned bare aluminum mirrors (Wilson 1999), along with simulated anti-reflection coating curves to match the specifications of the two wide field corrector lenses (a small overall effect). Measured curves were used for the dichroic, grism, camera coatings, and CCD quantum efficiency (QE). The manufacturer's curve for Denton FSS99 silver was used for the collimator coating. Internal transmission curves for the camera glasses were obtained from the manufacturer's data sheets. The fiber efficiency $(85 \%)$ is based on lab measurements, representing an average value for the fibers measured and assumed flat across the bandpass. ${ }^{37}$ An additional $3 \%$ loss is included for FRD beyond the $f / 4$ beam the collimator was designed to accept. Figure 12 shows the individual component efficiencies used for this model along with the total expected throughput. Peak efficiencies in the blue and red channels are $17 \%$ and $22 \%$, respectively.

\subsection{Mechanical Design \\ 2.4.1. Overview}

The twin spectrographs mount to the SDSS telescope Cassegrain rotator adjacent to the focal plane with sufficient separation between the spectrographs to allow routine installation and removal of the imaging camera and fiber cartridges; see Figure 13. Compared to a bench-mounted configuration this approach greatly reduces fiber length, and the associated throughput loss. It also eliminates potential adverse effects resulting

\footnotetext{
37 As per the Polymicro data sheet, the variation in transmission is only about
} $2 \%-3 \%$ over the SDSS bandpass. 


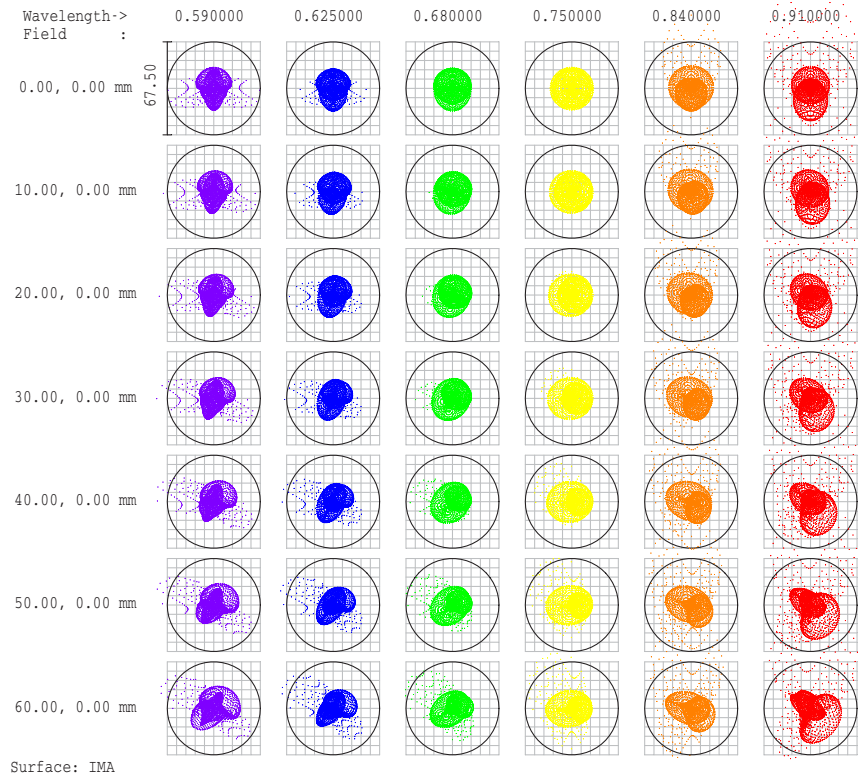

Figure 9. Spot diagram for the SDSS red channel. The average rms spot diameter is $21.5 \mu \mathrm{m}$ and the maximum rms diameter is $29.2 \mu \mathrm{m}$.

from the repeated bending of fibers. A consequence, however, is flexure induced motion of the spectra as the telescope tracks the sky, a problem that is mitigated by the use of a stiff, enclosed optical bench.

Each spectrograph has a mass of approximately $320 \mathrm{~kg}$. The overall length, width, and height are $2210 \mathrm{~mm} \times 1000 \mathrm{~mm} \times$ $570 \mathrm{~mm}$, respectively. The optical layout of the instrument lends itself to a simple mechanical design, having only a few mechanisms and only one actively controlled optic, the collimator. To ease assembly and testing, the fiber slithead and all refractive components (i.e., camera lenses, the dichroic, and grisms) are mounted without adjustment in logical sub-assemblies, relying solely on machining tolerances for placement. Shims are located at strategic locations where opto-mechanical tolerances are tighter than could be achieved with reasonable machining practices. This basic approach has produced a robust, reliable instrument that has been straightforward to maintain and upgrade. In the following sections we describe the mechanical design in more detail, with an emphasis on the opto-mechanical sub-assemblies.

\subsubsection{Optical Bench}

Figure 14 shows the spectrograph optical bench and the opto-mechanical subassemblies that interface to it. The optical bench is an enclosed aluminum (6061-T6) weldment with precision-machined interfaces to locate all five opto-mechanical subassemblies: the fiber slithead, the collimator, the central optics, and the red and blue channel cameras. The dual-channel optical layout naturally leads to the T-shaped configuration depicted in the figure. Both the red and blue cameras, as well as the collimator assembly, mount to exterior faces of the bench; one at the end of each leg of the T. The fiber slithead and the central optics assembly, which contains the dichroic and grisms, are mounted internally through access ports. At all of these opto-mechanical interfaces precision-machined reference datums and kinematic-locating features are employed to ensure accurate, repeatable alignment of the optical system. A shim at the collimator interface provides a one-time coarse adjustment to compensate for the as-built collimator radius of curvature.

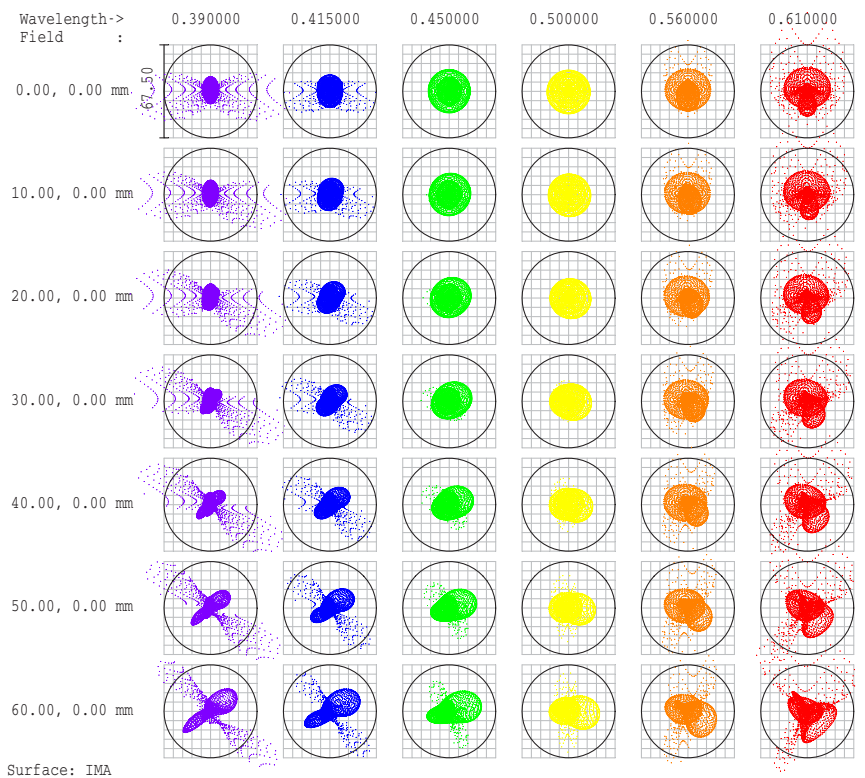

Figure 10. Spot diagram for the SDSS blue channel. The average rms diameter is $21.1 \mu \mathrm{m}$ and the maximum rms diameter is $27.3 \mu \mathrm{m}$.

Another shim where the slithead kinematic mount attaches to the bench provides a one-time adjustment to compensate for machining tolerances. Instrument control electronics and the CCD controller mount to exterior walls of the bench. The guide camera, located on only one of the two spectrographs, mounts externally as well.

The principal considerations in designing the optical bench were ease of assembly, precise optical alignment, and the requirement to minimize flexure-induced motion of the spectra as the telescope tracks the field. With a tall closed-end, boxsection design, internal and external gussets, and optimized placement of the mount locations to the rotator, flexure-induced motion of the spectra is kept to an acceptably low level during a typical exposure. Secondary considerations include the desire to reduce stray light from external sources and to keep exposed optics free of dust and debris.

\subsubsection{Collimator Mount}

The collimator is the only actively controlled optic in the spectrograph; it is adjustable in piston, tip, and tilt. Enabling these degrees of freedom serves multiple functions and eliminates the need for numerous mechanisms elsewhere within the spectrograph. Translating the collimator in piston adjusts focus in both channels, an adjustment that occurs routinely during operation to compensate for thermal drift, and flexure due to changes in telescope pointing and rotator angle. A pair of Hartmann doors located in front of the collimator permits shifts in focus to be measured rapidly. Tip/tilt adjustment allows the collimator to be precisely coaligned to the cameras, or at least to an average of the two camera axes. Thus, the center fiber can be positioned at the center of the detector in the spatial direction, and the central wavelength can be positioned at the center of the detector in the spectral direction. Lastly, variations in the pixel-to-pixel sensitivity in the spatial direction can be accurately removed by dithering (i.e., tilting) the collimator between successive flat-field exposures and processing the coadded image. This is necessary because the image of the fiber on the detector has a Gaussian PSF, and the spectrograph flexes as the telescope moves, so the fiber illumination pattern changes 


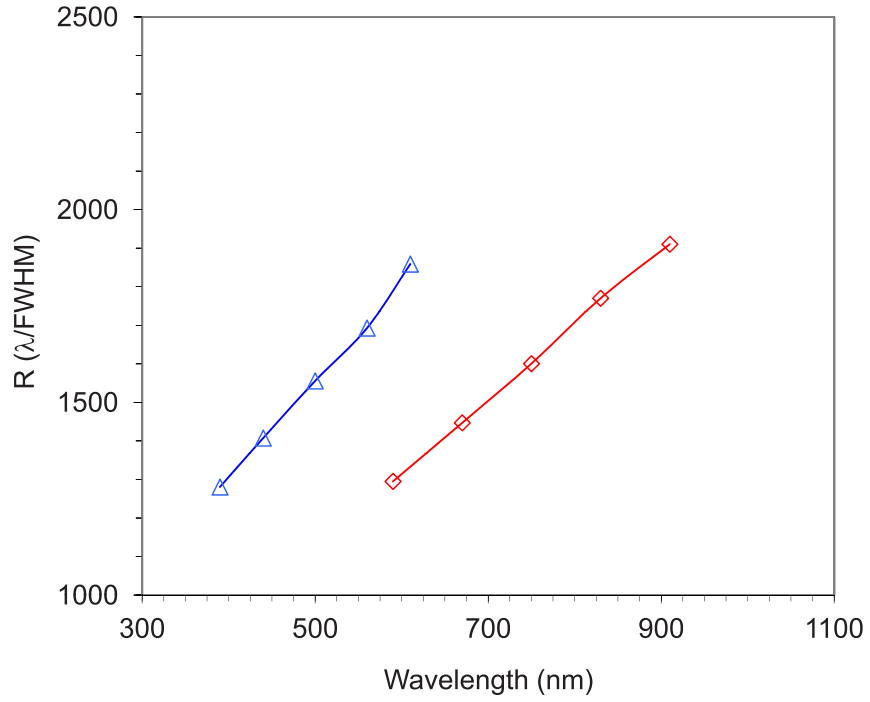

Figure 11. Predicted resolving power, $R$, as a function of wavelength for the SDSS spectrographs. Predictions are derived from the FWHM of simulated, uncollapsed images computed using Zemax. In reality, the resolving power of the instrument is determined using collapsed spectra, so the prediction here is on the low side. However, the Zemax analysis assumes perfect optics and alignment. For this reason the predictions have been based on uncollapsed spectra, which is the conservative approach. The expectation is that the actual resolving power will be somewhat better than shown here.

slowly. This does not matter if one has a uniformly illuminated flat, but is disastrous if one has little or poor calibration data where now there is a significant amount of flux because flexure has moved the fiber trace. Even if there is no flexure, the uniform flat provides better statistics across the fiber image. See Section 2.7 for a description of the flat-field dithering process.
Details of the collimator mount are depicted in Figure 15. The mirror is supported from the back surface, at its center, by a circular membrane flexure, which constrains the mirror in-plane but allows out-of-plane compliance for tip, tilt, and piston adjustment. Motion is provided by three linear actuators (Physik Instrumente model\# M-222.20 38 , which provide sub-micron resolution, more than sufficient focus adjustment, adjustment of the spectra on the CCD, and flat-field dithers. Each actuator connects through a spring-pre-loaded universal joint to an Alloy-42 steel pad bonded to the back of the mirror with $3 \mathrm{M} 2216^{39}$ two-part epoxy. Three limit switch assemblies behind the mirror limit travel to $\pm 3 \mathrm{~mm}$. The linear actuators, limit switches, and flexure are attached to a common mounting plate, which interfaces to the optical bench. Electrical connections from the actuators and limit switches are routed to a single connector on a custom printed circuit board mounted to the rear of the plate. A shim between the mounting plate and the bench facilitates one-time, gross focus adjustment. Accurate and repeatable assembly of the subsystem is made possible by two dowel pins (one round and one diamond shape) that engage hardened bushings pressed into the optical bench. Safe installation is facilitated by two handles for lifting, and four guide rods that flank the collimator ensure the collimator clears the bench opening as it is installed. A sheet metal cover surrounds the rear of the assembly, which protects vital components from physical and environmental damage, and reduces stray light. Finite element analysis conducted early in the design phase indicated that the distortion produced by gravity and the membrane load would be negligible.

\footnotetext{
38 Physik Instrumente, GmbH, http://www.physikinstrumente.com

39 3M, http://www.3m.com
}

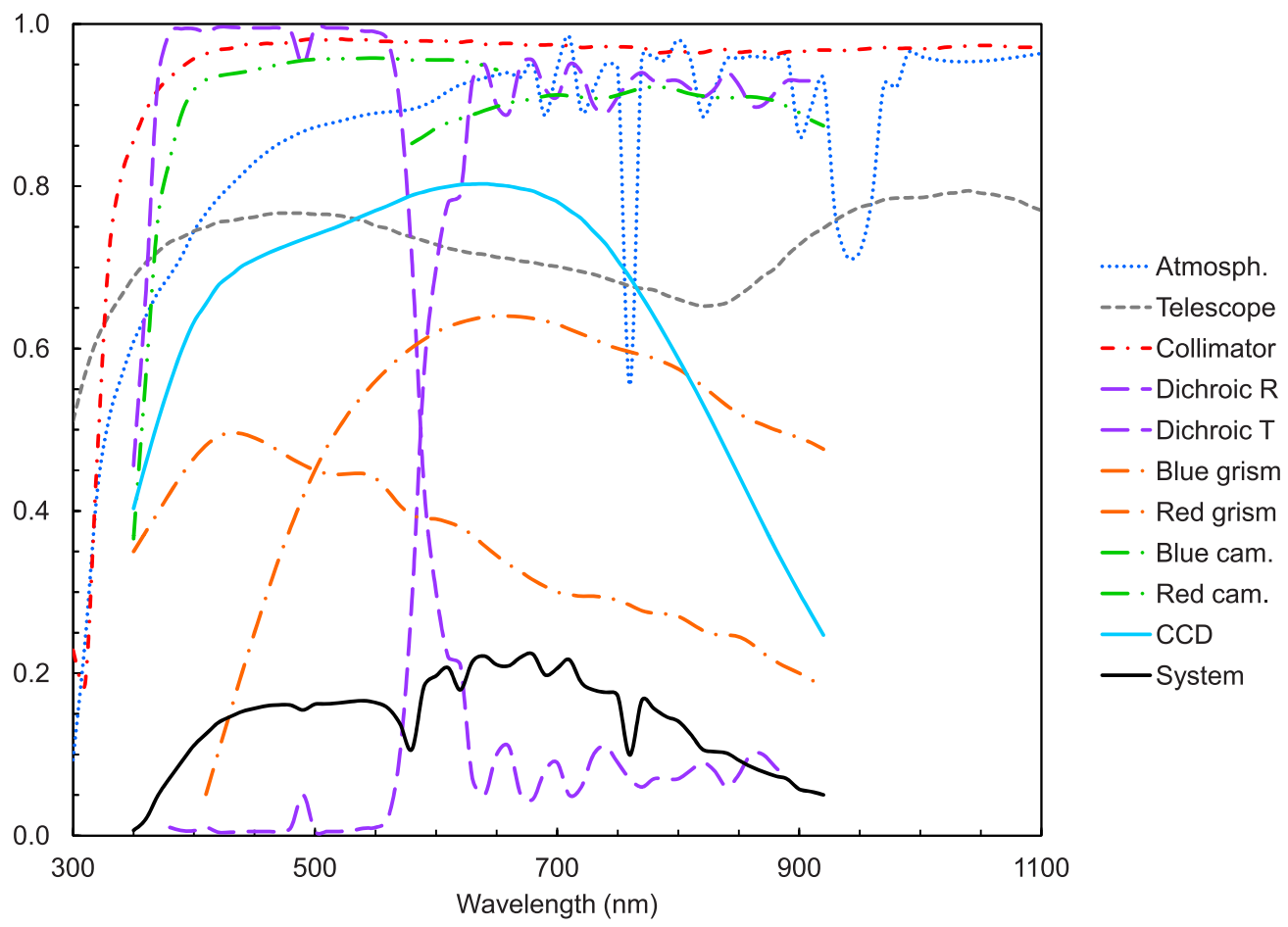

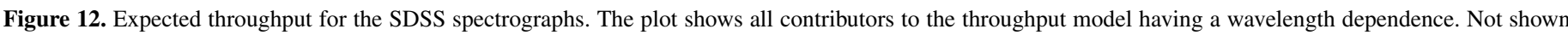

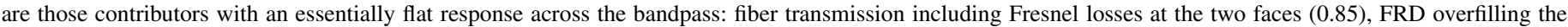

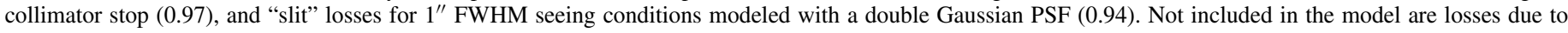

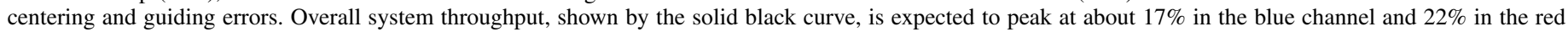
channel. 


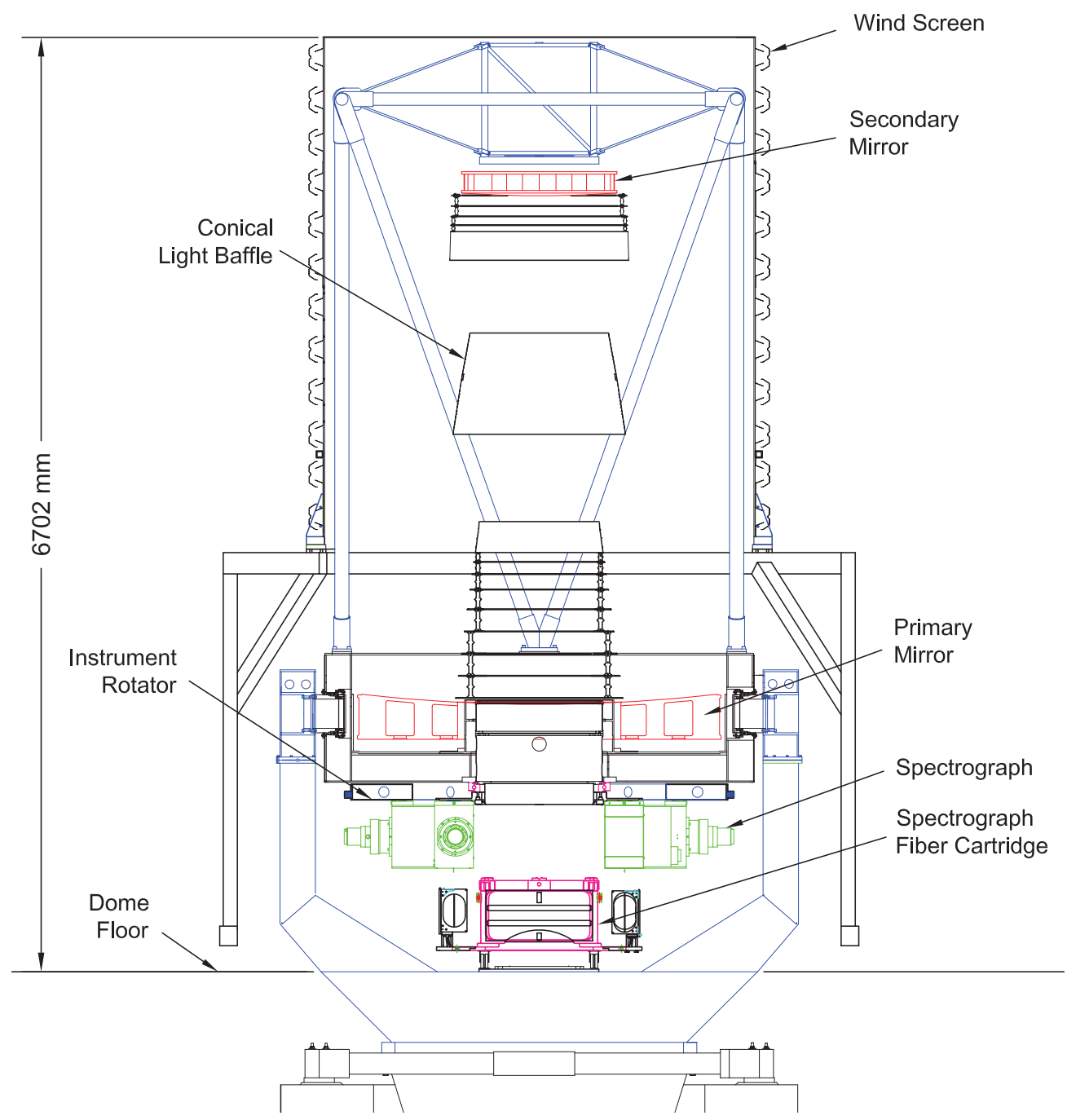

Figure 13. Front view of the SDSS telescope depicting the location of the spectrographs. Here a fiber cartridge is shown retracted from the spectrographs. The twin spectrographs, each with a mass of $320 \mathrm{~kg}$, mount to the back of the Cassegrain instrument rotator adjacent to the focal plane with sufficient separation between the spectrographs to allow routine installation and removal of the imaging camera and fiber cartridges.

\subsubsection{Hartmann Doors}

The Hartmann doors are a simple bi-fold design. Each door pivots about an upper and lower bushing pressed into the optical bench, and is driven by a $90^{\circ}$ pneumatic rotary actuator (Bimba Pneu-Turn model PT-006090-A1M ${ }^{40}$ ) located on the top surface of the bench. The doors are controlled by the spectrograph micro-controller and a bank of programmable solenoid valves (Clippard model number EMC- $08^{41}$ ) located in the spectrograph electronics box. Solid-state magnetic sensors (Bimba model number HSC-02) mounted to the actuators report the state of the doors (i.e., open or closed). Small manual flow control valves (Bimba model number FCP-1) at the inputs to each actuator set the speed of rotation.

\subsubsection{Shutter}

A shutter is required to set the exposure time for wavelength calibrations, flat fields, and science exposures. The shutter does not need to be fast or very accurate since science exposures include calibration standards that are used to determine the zero-point calibration at the time of the exposure. The minimum

\footnotetext{
40 Bimba Manufacturing Company, http://www.bimba.com

${ }^{41}$ Clippard Instrument Laboratory Inc., http://www.clippard.com
}

exposure time, $4 \mathrm{~s}$, is set by the arc lamps used for wavelength calibration. Flat fields require $30 \mathrm{~s}$ exposures. Science exposures are 15 minutes.

The spectrograph shutter is located just upstream of the dichroic on a dividing wall in the optical bench residing between the central optics and the slithead; see Figure 14. This location is ideal since the entire bandpass can be blocked by a single, relatively small shutter. The shutter is a black anodized aluminum sliding door driven by a double-acting pneumatic cylinder (Bimba model BRM-02x-DXP). Slots in the Delrin door frame guide the door, making a light-tight seal when the door is closed. An oval window in the frame, slightly oversized to the beam, serves as a light baffle. Like the Hartmann doors, the shutter is controlled by the spectrograph micro-controller and solenoid valves on the Clippard EMC-08 board. Solid-state magnetic sensors (Bimba model number HSC-02) mounted to the cylinder indicate the state of the door (i.e., open or closed). Small manual flow control valves (Bimba model number FCP-1) at the inputs to cylinder set the speed at which the door opens and closes.

The shutter is accurate to approximately $0.5 \mathrm{~s}$. Latency in the door motion due to temperature changes is responsible for most of the inaccuracy. 


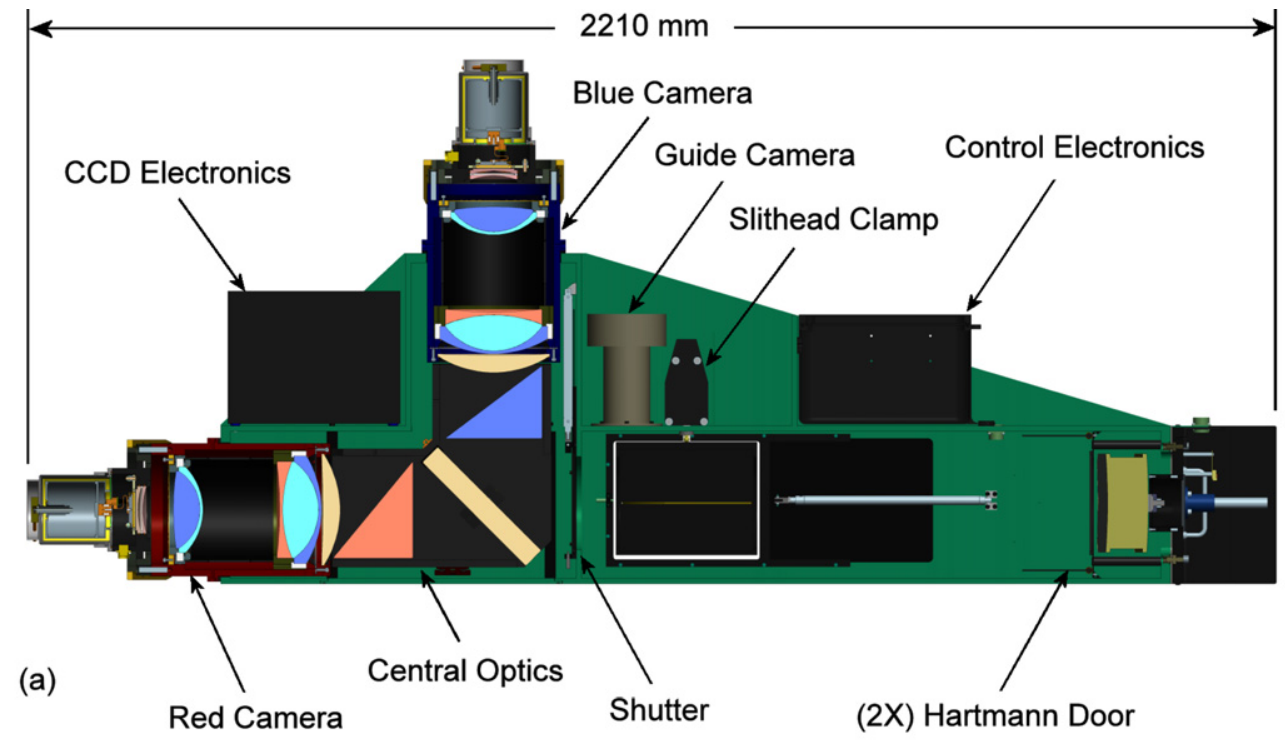

(3X) Spectrograph Mount

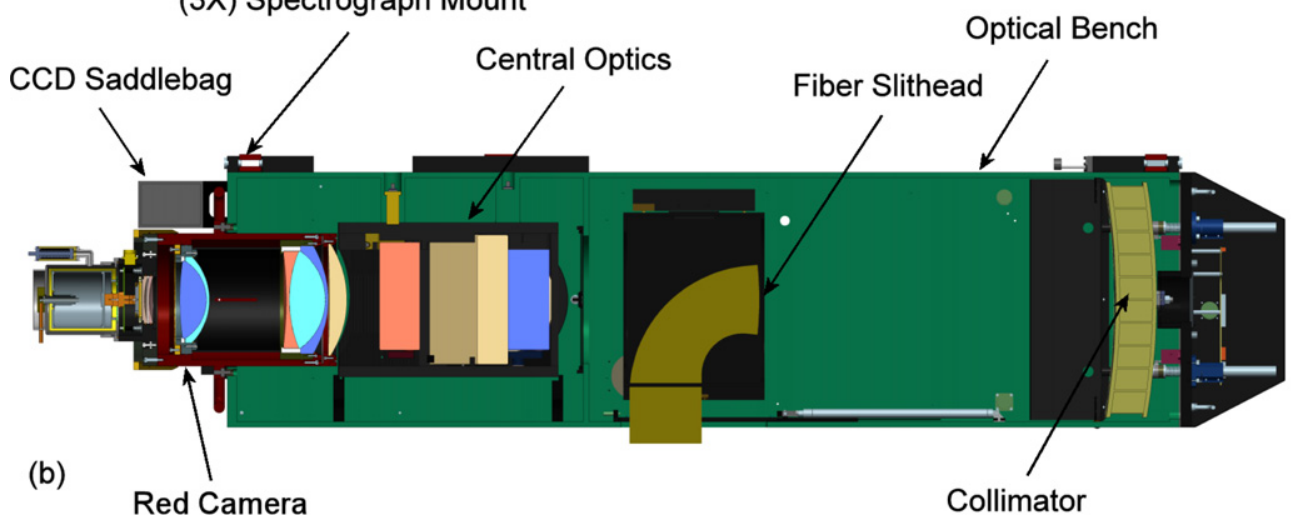

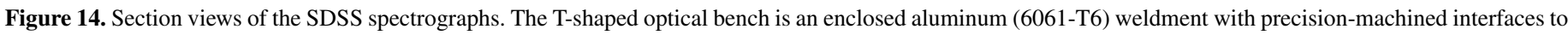

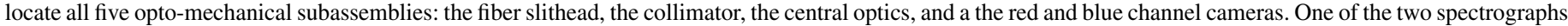

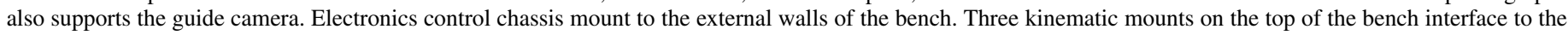
back of the Cassegrain instrument rotator.

\subsubsection{Central Optical Assembly}

The beamsplitter and grisms are mounted in a single optomechanical structure, the central optics assembly. The assembly is kinematically mounted inside the optical bench on three gusseted posts integral to the weldment and located on the top surface of the optical bench, thus the central optics assembly is suspended from the top of the bench. The faces of the three posts are precisely machined establishing a planar reference that is square to the collimator mounting surface and the two camera mounting surfaces. Two locating sleeves centered about two of the three posts establish the in-plane location of the assembly. These machined references precisely locate the assembly relative to the slithead, collimator, and cameras. A large port in the sidewall of the optical bench provides access for machining the post surfaces and installation.

Figure 16 shows the details of the central optics assembly. The dichroic and both grisms are each located, without adjustment, by six machined reference surfaces (Kapton tape covers each surface to avoid metal-to-glass contact). Spring plungers seat the elements against these surfaces accommodating differential contraction between the glass optics and aluminum structure. A three-point-contact Alloy-39 block bonded to the top of each grism spreads the vertical load applied by a single large plunger embedded in the top plate, and provides a convenient lift point for installing the grism. To minimize tolerance stack-up and improve placement accuracy, all but 1 of the 18 reference surfaces (6 for each of 3 optics) is machined into a single component, the base plate, which interfaces to the optical bench. The single remaining reference surface, which controls the tip of the beamsplitter, is located on the top plate. Black anodized surfaces and thin light baffles at the exit faces of the assembly serve to mitigate stray light. The entire assembly has a mass of $39 \mathrm{~kg}$.

\subsubsection{Camera Opto-mechanics}

The opto-mechanical design of the SDSS cameras, shown in Figure 17, was derived largely from the Norris spectrograph camera design (Cohen et al. 1988); a logical consequence of the two cameras having very similar optical designs, and the same optical designer, Harland Epps. The lenses and lens-groups are mounted in athermal cells. External reference surfaces on each cell are machined true to the opto-mechanical reference surfaces that locate each lens, thus establishing lens concentricity from cell-to-cell and accurate placement of the lenses along the 


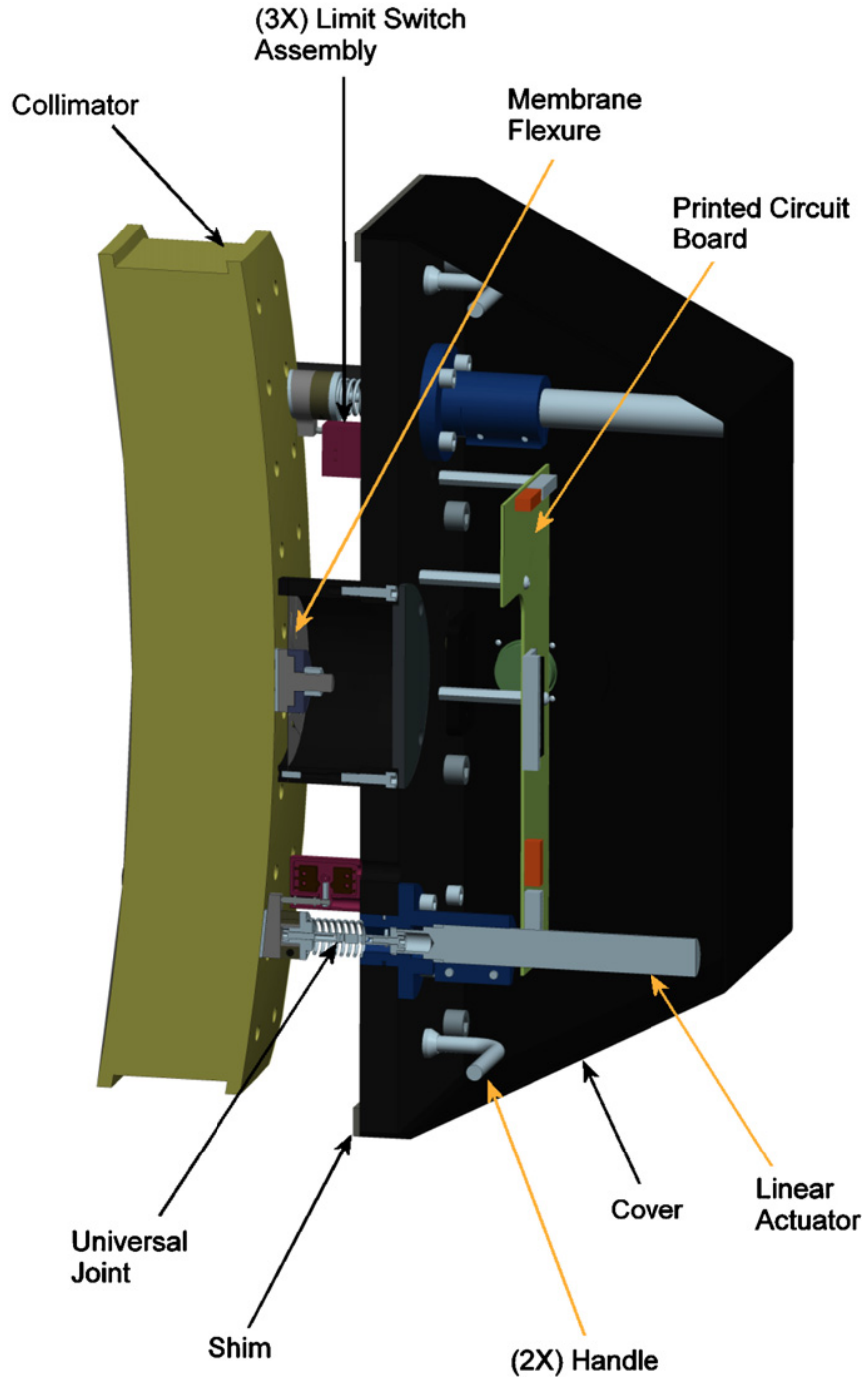

Figure 15. Collimator mount for the SDSS spectrographs. The mirror is supported from the back surface, at its center, by a circular membrane flexure, which constrains the mirror in-plane but allows out-of-plane compliance for tip, tilt, and piston adjustment. Motion is provided by three linear actuators, which provide sub-micron resolution, more than sufficient for focus adjustment, adjustment of the spectra on the CCD, and flat-field dithers.

optical axis. The singlet and triplet cells are bolted together in series and mounted in an aluminum barrel along with the doublet cell. A steel inner barrel meters the distance between the triplet and doublet, the doublet being pressed against the inner barrel by a compression spring assembly behind the cell. A thick shim between the singlet and triplet allows a one-time adjustment to compensate for machining tolerance stackup. The field flatteners are integral to the dewar, as described in Section 2.4.8.

The design and construction of the athermal cell design is best described as follows. A metal ring with an appropriate coefficient of thermal expansion is bored oversize to the lens diameter. Six glass-filled Teflon plugs are lightly pressed into a hole pattern circumscribing the lens bore. Here the rear two elements are located by a single set of plugs given the small edge thickness of the middle, calcium fluoride, element. These plugs are then bored on a lathe to a diameter slightly oversized ( $50 \mu \mathrm{m}$ on diameter) to the as-built lens diameter. In the same machining step, the remaining critical surfaces are machined, thus achieving lens concentricity (within the limits of radial clearance), location along the optical axis, and perpendicularity to the optical axis. The plug diameter is calculated such that

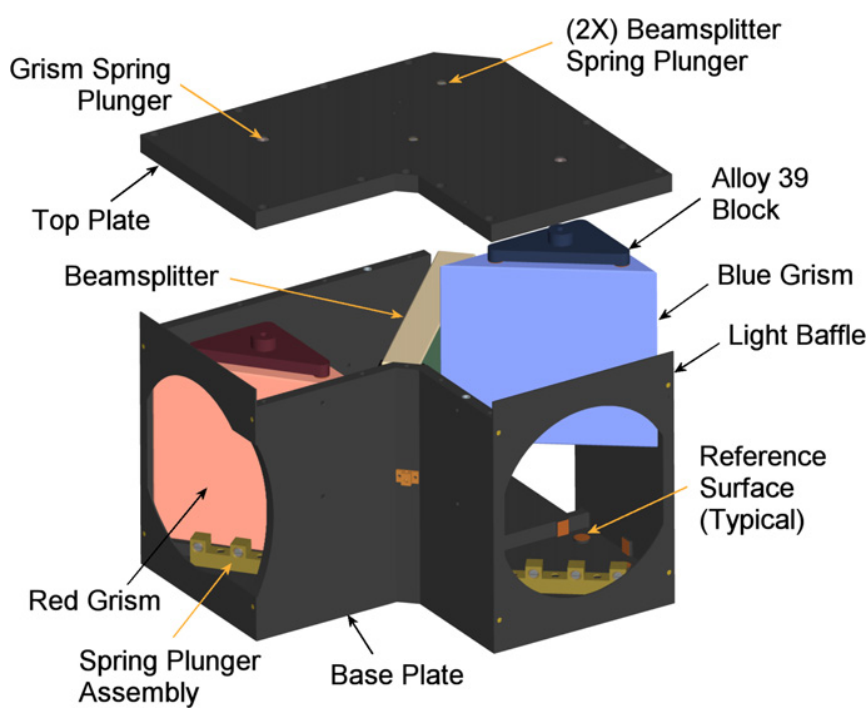

Figure 16. Exploded view of the Central Optics Assembly. The dichroic and both grisms are each located, without adjustment, by six machined reference surfaces. Spring plungers seat the elements against these reference surfaces and accommodate differential contraction between the glass optics and aluminum structure. Kinematic mounting interfaces on the bottom of the base plate locate the assembly inside the optical bench.

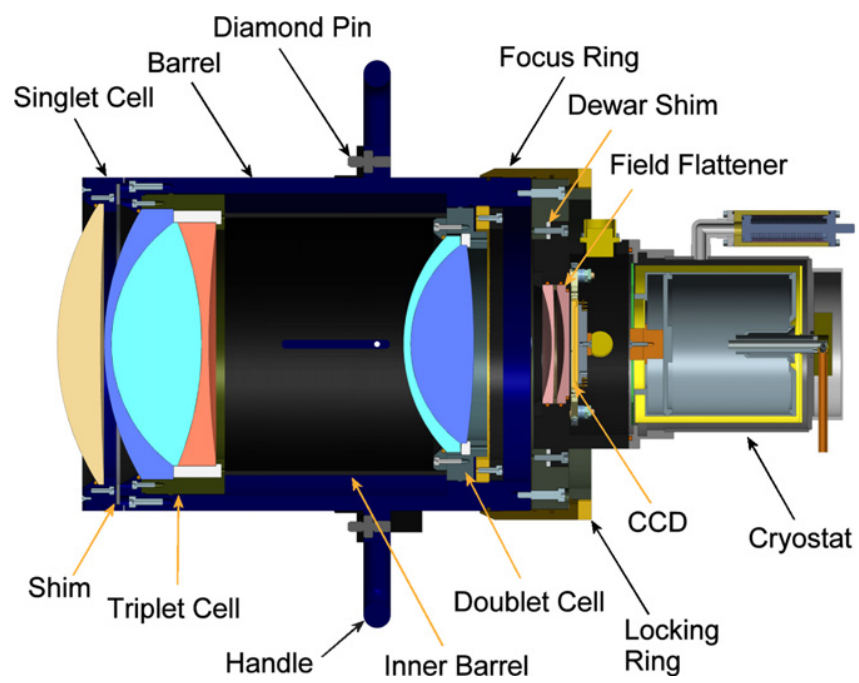

Figure 17. Cross section of the SDSS blue camera highlighting the optomechanical design details. The opto-mechanical details of the red camera are identical. The design was derived largely from the Norris spectrograph camera design. The lenses and lens-groups are mounted in athermal cells. External reference surfaces on each cell are machined true to the opto-mechanical reference surfaces that locate each lens, thus establishing lens concentricity from cell-to-cell and accurate placement of the lenses along the optical axis. Manual focus adjustment is provided by a threaded focus ring at the back of the camera barrel. This adjustment is used to compensate for small focus drifts and to ensure the two channels are parfocal.

the net change in the diameter of the finished bore is less than the diametrical clearance to the lens over the temperature range of interest, given the coefficients of expansion for the metal ring, the Teflon plug, and the glass lens. Where lens groups are packaged in a single cell, multiple sets of plugs are used. For the SDSS cameras, 6061-T6 aluminum was used for the singlet and doublet cells, and 330 stainless steel was used for the triplet cell. A Kapton shim rests between the lens locating face and the metal cell. An O-ring in the retaining ring contacts the first lens surface, providing force to seat the lens and compliance to 


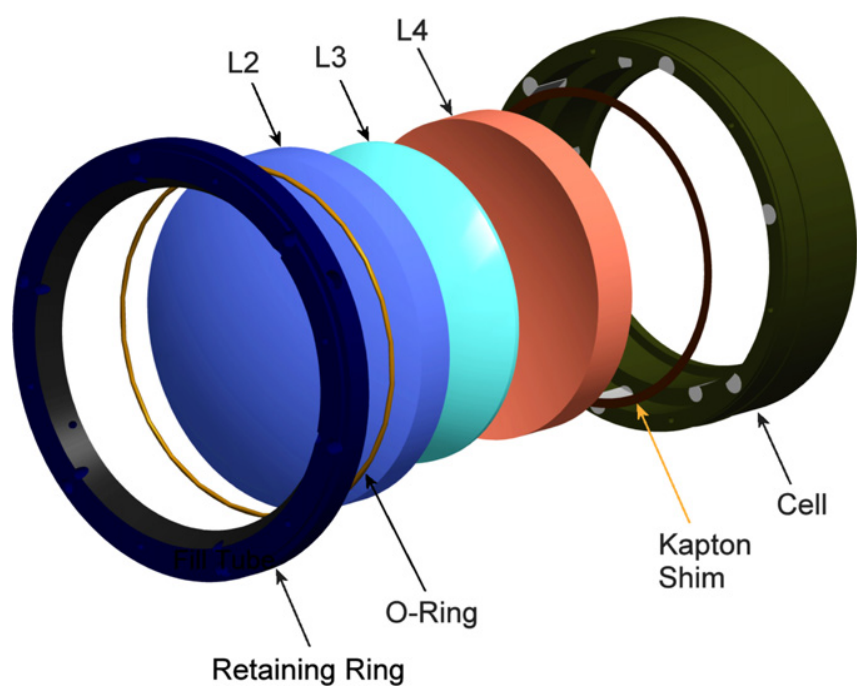

Figure 18. Exploded view of the SDSS blue camera triplet. The athermal cell design consists of a type 330 stainless steel ring with six glass-filled Teflon plugs lightly pressed into a hole pattern circumscribing each lens bore. Here the rear two elements are located by a single set of plugs given the small edge thickness of the middle, calcium fluoride, element. These plugs are then bored on a lathe to a diameter slightly oversized $(0.05 \mathrm{~mm}$ on diameter $)$ to the as-built lens diameter. The net change in the diameter of the finished bore is less than the diametrical clearance to the lens over the temperature range of interest, given the coefficients of expansion for the metal ring, the Teflon plug, and the glass lens. An o-ring in the retaining ring contacts the first lens surface, providing force to seat the lens and compliance to accommodate differential expansion.

accommodate differential expansion. A rendered image of the triplet cell depicting these details is shown in Figure 18.

A mounting flange on the camera barrel (located at the camera center of gravity) provides the interface to the optical bench. Two pins (one dowel pin and one diamond pin) in the face of the flange engage two steel bushings in the bench, ensuring repeatable placement. Handles on the flange, and a separate, detachable guide-rail system, which bolts to the optical bench, facilitate safe removal and installation of the camera. The entire camera mass (including the dewar) is $40 \mathrm{~kg}$.

Camera focus is achieved by translating the dewar, which houses the detector and the two field flattening lenses, and attaches to a flange at the rear of the camera barrel. A shim between the dewar and flange provides a one-time coarse focus adjustment. Small focus adjustments to compensate for seasonal temperature variations are affected by turning the focus ring threaded onto the rear of the camera barrel. Turning the ring translates the dewar flange, which is pre-loaded against the ring by eight compression springs in the rear face of the camera barrel. Two guide pins, also in the rear face of the barrel, engage two linear bearings in the flange providing smooth axial motion with negligible lateral motion. The large diameter of the focus ring combined with a fine 32 thread-per-inch pitch allow focus resolution of roughly $2 \mu \mathrm{m}$. Marks engraved in $1^{\circ}$ increments on the outer diameter of the ring provide a convenient method for metering adjustments.

\subsubsection{Dewars}

The CCD is housed in a dewar and cooled using liquid nitrogen $\left(\mathrm{LN}_{2}\right)$; see Figure 19.

The front half of the dewar contains the last two elements of the camera (i.e., the field flatteners), the detector, and the preamp board. O-rings locate the lenses on-axis and provide radial compliance for what is a small differential thermal contraction

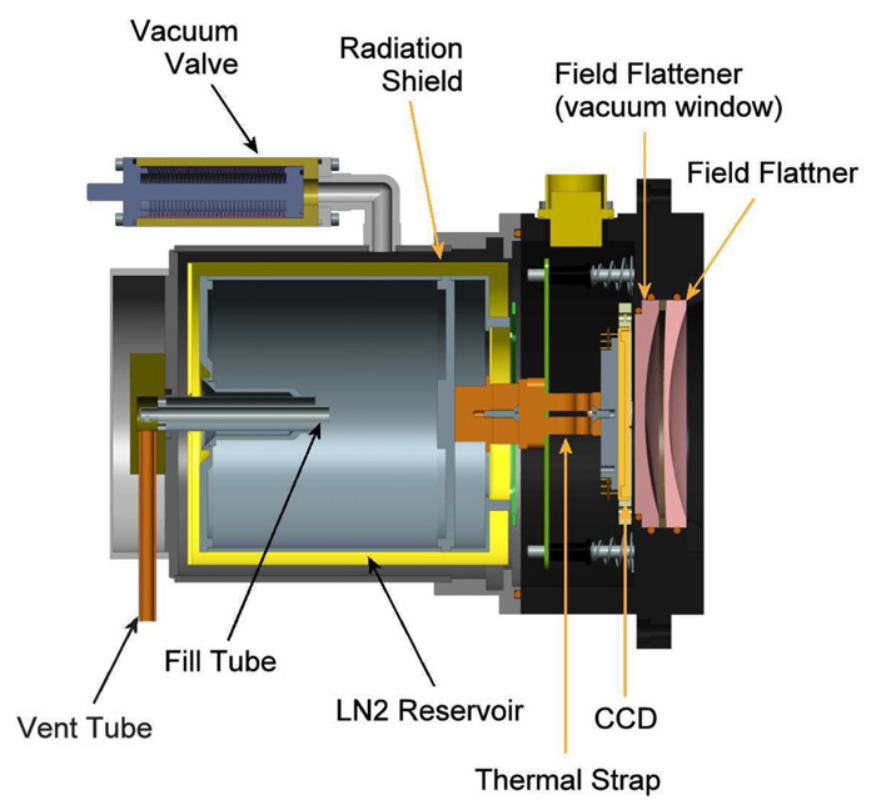

Figure 19. Cross section of the CCD dewar. The liquid nitrogen dewar contains the last two elements of the camera (i.e., the field flatteners), the detector, and the pre-amp board. Location of the CCD is adjustable in five degrees of freedom for centration, piston, tip, and tilt. In operation the total dewar heat load is $4 \mathrm{~W}$. An autofill system fills the dewar at roughly three hour intervals from a 10 liter storage dewar adjacent to the spectrograph.

over the operating temperature range. Location of the CCD is adjustable in five degrees of freedom for centration, piston, tip, and tilt. The detector columns are aligned to the slit by rotating the entire dewar relative to the back of the camera.

The rear half of the dewar contains the $\mathrm{LN}_{2}$ reservoir and is bolted to the front half of the dewar, a logical arrangement whereby the front half containing the optics, detector, and electronics can be assembled independent of the rear half containing the liquid nitrogen tank. A flexible copper braid provides the thermal connection between the reservoir and the detector. The space surrounding the reservoir and the remainder of the internal volume extending up to the rear of the last field flattener is evacuated. Detector temperature is controlled to $180 \pm 0.5 \mathrm{~K}$ using a small heater located on the cold-finger attached to the rear of the detector. The $\mathrm{LN}_{2}$ reservoir is filled from the rear of the dewar through the center of a coaxial tube located on the reservoir axis and penetrating to the middle of the volume; this allows half of the 0.6 liter volume to be filled with liquid, regardless of gravity vector orientation. Gaseous boil-off vents through the outer tube.

The thermal design of the dewar is well optimized. The liquid nitrogen reservoir is fabricated from stainless steel and is well isolated from its surroundings. Aside from the detector cold strap, the only conductive paths to the reservoir are the thinwalled stainless vent tube and the G10 spider that provides radial support at the front end of the reservoir. Polished and gold-plated surfaces on the exterior of the reservoir and the radiation shield minimize radiative heat load. In operation the total dewar heat load is $4 \mathrm{~W}$ and the hold time is approximately three hours.

\subsubsection{Dewar Autofill System}

Camera dewars are filled automatically using a liquid nitrogen autofill system arranged in the following manner. A single 180 liter dewar resides on the telescope platform and is 
connected, during the day only, to two 10 liter intermediate dewars mounted on the instrument rotator; one next to each spectrograph. Each 10 liter dewar feeds the two camera dewars on the adjacent spectrograph. Cryogenic solenoid valves $\left(\mathrm{ASCO}^{42}\right.$ Red Hat series), one per dewar, control the flow. The intermediate dewars have sufficient capacity to operate through the night without refilling.

Dewar fill is initiated by a timer in the camera electronics that is triggered every hour. (Filling every hour maintains a more even fill level minimizing temperature variations in the dewar.) Termination is controlled by a simple analog circuit that uses a thermistor on the vent line to sense the liquid overflow when the dewar is full. With this arrangement, both spectrograph camera dewars start filling at the same time, but terminate independently depending on the amount of $\mathrm{LN}_{2}$ remaining at the start of the fill, and variation in parasitic losses in the plumbing. Camera dewars may be filled during observations without any impact on the science. It typically takes six minutes to fill the dewar once the fill cycle is initiated.

For the 10 liter intermediate dewars, filling is initiated and terminated using the same analog circuit scheme described for the camera dewars. However, two sensors are used; one located in the tank to sense when the tank is empty, the other located on the vent line to sense when it is full. Like the camera dewars, the 10 liter dewars are filled to half capacity to avoid spillage as the telescope slews. Again, the 10 liter intermediate dewars are only filled when the telescope is not in operation. During operation they are disconnected from the 180 liter supply dewar. Logic in the autofill circuitry prevents the 10 liter empty sensor from triggering a fill if the lines are not connected. Similarly, interlocks prevent the telescope axes from going live if the 180 liter supply line is connected to the intermediate dewars.

\subsection{Flexure}

The variation in gravity load as the telescope tracks the sky causes gravity-induced deformations within the instrument to change with time and these changes lead to motion of the spectra on the detector during an exposure. Motion of the spectra can degrade resolution and increase cross-talk between the spectra. For this reason, the optical bench and the opto-mechanical assemblies (slithead, collimator, central optics assembly, and cameras) were designed to be extremely stiff.

A goal was set to limit flexure to 0.3 pixels in one hour, or $15^{\circ}$ on the sky. This goal stems from the experience that one could reliably find the centroid of an unresolved emission line (a calibration arc line) to $1 / 10$ the slit width, or 0.3 pixels in this case. So assuming a set of exposures would last an hour (three 15 minute science exposures, plus 15 minutes for field acquisition and calibration), it would take a shift of 0.3 or more pixels before it would be noticed. The 0.3 pixels translates to, roughly, $0.3 \AA$ in the blue camera, or roughly $20 \mathrm{~km} \mathrm{~s}^{-1}$ in redshift. Since galaxy internal velocity dispersions are on the order of $200 \mathrm{~km} \mathrm{~s}^{-1}$, a galaxy absorption line would be 3 or $4 \AA$ wide and just resolvable by the spectrograph. Therefore, flexing 0.3 pixels would just barely be detectable in a single galaxy absorption line. Now it is the case that with night sky lines for reference, as well as the lines from the calibration lamps, this amount of flexure would not be a problem for the actual velocity measurements for high enough $\mathrm{S} / \mathrm{N}$. However, the smearing could make sky subtraction a problem. Sky subtraction, at that time, was a much more difficult problem.

\footnotetext{
$\overline{42}$ ASCO Valve, Inc., http://www.ascovalve.com
}

The 0.3 pixel budget was divided equally between each of three subsystems: the slithead, the collimator, and the optical bench; each being allowed to contribute no more than 0.1 pixel shift in one hour. It was assumed, based on the details of the design, that the cameras and central optics assembly were rigid. It was also assumed, based on the designs, that the slithead and collimator would not exceed the 0.1 pixel allowance. Hence a detailed analysis was not performed on these two subsystems. The collimator was analyzed for surface figure deformation as a function of gravity for the described mounting constraints, but these models assumed rigid actuators, something we knew was not true but was sufficient for the purpose of quantifying the change in surface figure.

The optical bench, however, did receive scrutiny. The primary concern being the deformation in the relatively thin plate structure with multiple heavy optical assemblies attached to it. To assess the stiffness of the optical bench a finite element study was conducted in collaboration with Swales Aerospace Corporation. The goal of the analysis was to quantify the shift in the spectra at the detector for a change in gravity vector orientation of $15^{\circ}$.

The results showed that flexure in the bench is influenced largely by the placement of the three kinematic support points that attach the instrument to the rotator, as well as the thickness of the bench top plate. The optimal placement of the supports was determined to be at the edges of the bench; this set the final location for the mounts. In addition, the region of the bench around the mount points was stiffened by a thick plate, which became integral to the mount itself. The results indicated that with this configuration, and for rotations of $15^{\circ}$ about the red camera axis, the shift in the spectra would be less than 0.1 pixels in both channels.

Details on flexure performance can be found in Section 4.3.

\subsection{Detectors, Electronics, and Data Acquisition}

\subsubsection{Detectors}

The CCD detectors are thinned Tektronix/SITe SI424A $2048 \times 2048$ devices with square $24 \mu \mathrm{m}$ pixels. The readout is done through two amplifiers at opposite ends of a single serial register. Readout noise is about 5 electrons and full well is about 150,000 electrons. A remarkable characteristic of these devices is a high QE in the blue spectral region.

\subsubsection{Detector Readout}

The readout electronics are essentially identical to those used in the SDSS camera, which were described in detail in Gunn et al. (1998). In brief, the electronics consist of an in-vacuum preamp/clock driver using Burr-Brown OPA62 $7^{43}$ FET-input low-noise operational amplifiers and a set of solid-state switches for DC restoration and to generate the CCD clocks from supplied rail voltages and CMOS clock signals. This unit is physically and electronically identical to the ones used in the camera. The voltages and signals come to the dewar over a 37 wire cable, and the video is transmitted over a 10-wire cable. These cables are routed to a saddlebag, which is mounted to the optical bench and carries three cards: (1) a bus receiver, which receives clock signals over an RS-485 bus serving both dewars on a single spectrograph and produces CMOS signals for the dewar and the other saddlebag cards; (2) a power distribution

\footnotetext{
$\overline{43}$ Now manufactured by Texas Instruments, http://www.ti.com
} 
board, which regulates and filters incoming DC power for distribution to the saddlebags and dewar, and contains some of the temperature regulation circuitry; and (3) a complex eight-layer signal-chain/bias board, which has circuitry on one side to generate all the needed rail voltages and on the other to implement CDS processing of the CCD video, digitize it, and drive an RS-485 output line to the controller. With the exception of some component values, these cards are identical to the ones used in the camera.

The RS-485 bus signals and the RS-485 digitized video to/from each dewar/saddlebag are carried to a single controller chassis for each spectrograph on two cables, the RS-485 on a standard 50 pin SCSI cable and the video/ADC signals on a ribbon cable terminated with DB-15s. The controller houses power supplies, using boards essentially identical to those used for the camera, the TDS Forth microprocessors ${ }^{44}$ used for all control functions and to generate the CCD clock waveforms, RS-485 driver/receiver circuitry, and circuitry to drive the FOXI fiber transmitters, ${ }^{45}$ which send the (now multiplexed) digital data from the instrument to the data acquisition hardware approximately a hundred meters away in the operations building. The camera uses two micros, one for executive control functions and one for waveform generation, but in the somewhat simpler spectrograph environment these functions are combined. Again, see the camera paper for details.

\subsubsection{Data Acquisition}

The data acquisition hardware for the spectrograph CCDs is a clone of the photometric camera system. The data are received on FOXI receivers, demultiplexed, and stored on a "pool" SCSI disk by a Motorola MVME167 single-board computer. Transfers from this VME backplane to the Linux host are handled by a custom interface designed and built at Fermilab.

On the Linux host, the observer's program (SOS) actuates the system through remote procedure calls (RPC) over the Ethernet. The commands are few and simple, only those required to prepare the CCD, expose, and read out the data. Once the data are off the CCDs and in the data system pool, the images are downloaded over the high speed VME link to the observer's workstation where they are written to disk. At the end of the night, the SDSS data was archived and sent to Fermilab. A typical night of observing produces $\sim 1.5 \mathrm{~GB}$ of data.

The drilling database is the list of objects on a plug-plate. It includes the name and drilling coordinates of each object and the field coordinates ( $\alpha, \delta$, equinox) of the plug-plate. This information is needed at observing time so the telescope can be pointed and the plug-plate ID can be incorporated into the data file. This database is generated at Fermilab from the photometric imaging data and is delivered on the internet to the observer's workstation at Apache Point well before the observations.

As described in 2.2.8, the plugging station is equipped with a device to map the plug-plate locations to the slithead after plugging is finished. The plugging database information is written directly to the observer's workstation disk at plug time. The name of this file is then merged with the CCD data before it is written to disk as extended FITS keywords for each exposure (see footnote 30 ).

\footnotetext{
44 Triangle Digital Systems, http://www.triangledigital.com

45 FOXI transmitters were provided by Fermi National Accelerator Lab.
}

\subsubsection{Spectrograph Control}

Spectrograph operations are controlled from a single program (called SOP) running on the observer's Linux workstation. This program, the observer's sole interface to the spectrographic system, is a clearinghouse for observing commands that translates observer's requests into the commands required by the independent subsystems used for spectroscopy. These systems include: spectrograph microprocessor, telescope and guider, CCD data acquisition system, drilling database, and plugging station.

The spectrograph mechanical controls are handled by a Z-World Little Giant Z180 microprocessor board. This processor communicates with the observer's workstation via RS-232A serial commands sent through an Ethernet terminal server located at the telescope. This processor handles all motion control (shutters, Hartmann masks, collimator tip, tilt, and focus) and also reports ambient and instrument temperatures for focus compensation.

The observer's software communicates fully with the telescope control system via an Ethernet telnet connection. When a new plug-plate is locked on the telescope, the spectrograph relays the plug-plate ID to the observer's software, which looks up the coordinates in the plug-plate database. The observer's software commands the telescope to move to the mid HA position for flat field and wavelength calibration, then to the field for precise positioning, scaling, and focusing. When these operations are finished, the observer's software starts the guider, opens the shutters, and begins observing.

\subsection{Calibration}

Calibration for the spectrographs, both flat-fielding to tie the responses of the fibers together for flux calibration, and the use of spectral lamps to provide wavelength calibration, are provided as part of the telescope structure. The screens for these functions as well as the lamps are mounted on the windbaffle structure, which serves as a dome/enclosure for the SDSS telescope (see, e.g., Gunn et al. 2006).

At the top of the windbaffle structure is a mechanism incorporating eight lightweight aluminum-honeycomb sectorshaped panels mounted on shafts and driven by DC gearmotors. These panels can be closed to cover the annular aperture of the telescope or opened to allow observing. The bottoms of these panels are coated with a special wide-spectrum Lambertian paint (PolarKote ${ }^{46}$ ), providing a highly uniform diffuse reflective surface.

The panels are illuminated in quarters by a bank of three projectors in each corner of the windbaffle structure just above the central structure which supports the primary mirror and the altitude bearings. The projectors output a quarter-annulus, which fills the pupil of the telescope and avoids illuminating the secondary structure directly. One of these units in each of the three-projector assemblies is illuminated by a $3100 \mathrm{~K}$ quartz-iodine lamp, ${ }^{47}$ and is used for flat fields; another houses a mercury-cadmium lamp, ${ }^{48}$ which has a moderately rich emission spectrum in the blue and green, and the last houses a

\footnotetext{
$\overline{46}$ Manufactured by Light Beam Industries LLC, http://www.lightbeaminc.com

47 Phillips Projection Lamp, Type 7027, model\# 409829, supplied by Specialty Bulb Co. Inc., http://www.bulbspecialists.com

48 Osram HgCd Lamp, model\# HgCd/10, supplied by Specialty Bulb Co. Inc., http://www.bulbspecialists.com
} 
neon lamp, ${ }^{49}$ which is used primarily to calibrate the red region of the spectrum.

The flat-field lamp is fitted with a combination of glass Schott filters (2 mm FG3 $+2 \mathrm{~mm} \mathrm{BG14})$ in an attempt to mitigate the very rapid falloff of the lamp spectrum to the blue, so that reasonable flat fields can be obtained with good $\mathrm{S} / \mathrm{N}$ in one exposure with no danger of saturation. This approach was moderately successful, but at the expense of producing a rather "lumpy" spectrum.

Since the spectrographs are mounted on the telescope, they flex, and so it is necessary to be careful about flat fields. Given the very large spatial modulation of brightness on the CCD, if the image of a fiber shifts 1 pixel during an exposure, the registration of the object spectrum and the calibration flat field drift out of alignment. Hence, very little flexure could be tolerated. For this reason, special flat fields are occasionally recorded by dithering the collimator mirror slightly in angle along the spatial direction. Doing so produces wider traces than the normal flat field exposures, thus determining pixel-to-pixel variations on the detector, and relaxing the flexure tolerance in the spatial direction. One can then properly calibrate the pixel-response during science exposures even if the spectrum moves slightly due to flexure.

In practice, this process of flat-field dithering consists of 20 successive exposures at various tilts of the collimator mirror. The sequence generates a map of flat-field projections offset by 0.6 pixels between exposures. Traversing a span of 12 pixels, about four fiber widths, these dithered flats therefore sample the detector response over the entire spatial direction.

\subsection{Guiding}

Guiding for the SDSS spectrograph is accomplished by the use of coherent imaging fiber bundles, which are connected to the plug-plates at the locations of guide stars using ferrules, which are externally identical to the science fiber ferrules. The imaging bundles used in SDSS-I and SDSS-II were Sumitomo ${ }^{50}$ coherent fibers of two sizes, which projected to $7^{\prime \prime}$ and $11^{\prime \prime}$ in the focal plane. There were nine $7^{\prime \prime}$ bundles and two $11^{\prime \prime}$ bundles. The larger ones were primarily intended for acquisition of the guide stars, although they were also used for normal guiding as well after all the stars were centered.

The guide fiber ferrules were modified slightly for this purpose; it is necessary, clearly, to orient the guide fibers rotationally so the guider will know what to do when a star is off-center. This is accomplished by mounting a collar with a small pin on the exterior diameter of the ferrule. A hole, smaller than the ferrule holes, receiving the pin is drilled into the plate at the time it is drilled, and the plate pluggers rotate the guide ferrule to drop the pin into the hole. The guide fibers are not randomly plugged, but assigned to marked holes in the plate.

The guide fibers are routed to 11 holes in a small block into which they are cemented and polished. That block is imaged through a back-to-back pair of $50 \mathrm{~mm} f / 1.2$ Nikon camera lenses, with a $2 \mathrm{~mm}$ Schott BG38 filter in the parallel beam between them, onto the CCD in a Roper Scientific (Photometrics) SenSys camera. ${ }^{51}$ The CCD is a Kodak KAF0401E, which has an $768 \times 512$ array of $9 \mu \mathrm{m}$ pixels. Detector readout was set in a bin-by- 2 mode, which resulted in $18 \mu \mathrm{m}\left(0{ }^{\prime} .3\right)$ pixels. The

\footnotetext{
49 Osram Neon Lamp, model\# Ne/10, supplied by Specialty Bulb Co. Inc., http://www.bulbspecialists.com

50 Sumitomo Electric Lightwave Corp., http://www.sumitomoelectric.com

51 Roper Scientific, Inc., http://www.roperscientific.com
}

camera has a thermoelectric cooler, which limits the CCD temperature to $10^{\circ} \mathrm{C}$, but is otherwise not temperature controlled. The camera came equipped with a SCSI interface, and was connected to an Apple Macintosh computer with a commercial SCSI-to-fiber-to-SCSI interface.

The images were buffered on the Mac and sent by fiber Ethernet to the control room where they are received and processed by the Linux SOP computer. A PID servo was implemented in that machine to generate correction signals to the telescope control computer. Display code to allow the observers to monitor the performance of the guider, either by generating basically a raw image of the fiber block or a display in which the images of the fibers are placed approximately where they are on the sky and oriented properly, was written.

The performance of the system was never completely satisfactory; the CCD was (essentially) uncooled, was noisy, had many dark defects, and its mounting was not mechanically stable. Halfway through the survey new code was written that handled centroiding properly even if the star was at the edge of the fiber image, and explicitly used dark frames and flat fields to correct the raw images. This enhancement helped, but a new and better camera was clearly needed, which did not arrive until the beginning of the BOSS survey.

\section{BOSS UPGRADE DESIGN}

The SDSS camera and spectrographs were operated for from 2000-2005, followed by a three-year extension known as SDSS-II. In 2005, the Astrophysical Research Consortium, which owns and operates the Apache Point Observatory, put out a call for proposals to operate the $2.5 \mathrm{~m}$ Sloan telescope and its instruments after 2008. SEGUE-II, a one-year extension of the Sloan Extension for Extra-Galactic Understanding and Exploration (SEGUE; Yanny et al. 2009) was approved for one observing season to use the SDSS spectrograph from mid2008 to mid-2009. In response to this announcement, the BOSS (Schlegel et al. 2009; Dawson et al. 2013) was also proposed and awarded five years of dark observing time beginning in 2009. The primary scientific objective proposed by the BOSS project was to map the baryon acoustic peak to redshift $z=0.7$ with percent-level precision. In addition, BOSS would pioneer a new technique to detect large-scale structure in the spectra of distant quasars by observing the so-called Ly $\alpha$ forest, a series of absorption features caused by intervening clouds of hydrogen gas.

To achieve the BOSS scientific goals, a major upgrade of the SDSS spectrographs was required. In particular, BOSS needed a higher multiplex factor in the number of fibers per exposure, and higher optical throughput. To achieve this, the BOSS proposal included plans to build a new fiber system and to upgrade the optical system and cameras of the original SDSS spectrographs. In this section we describe the requirements for BOSS and the hardware upgrades to achieve the new performance.

\subsection{BOSS Requirements}

The BOSS science goals require spectra of approximately 1.35 million luminous galaxies as faint as $i=19.9$, and 160,000 quasar spectra $(g<22)$, over $10,000 \mathrm{deg}^{2}$, in five years. Compared with SDSS, the BOSS spectrographs must obtain $35 \%$ more spectra per unit time for objects that are more than a magnitude fainter. While most of the SDSS spectrograph is portable to the BOSS requirements, higher multiplex factor and throughput necessitated changes to the original design. Here we 
summarize the requirements that dictated the upgrades for the BOSS spectrographs.

\subsubsection{Number of Fibers}

To achieve a percent-level precision measurement of the cosmic distance ladder, BOSS requires redshift measurements of approximately 1.35 million luminous galaxies to $z=0.7$ over $10,000 \mathrm{deg}^{2}$. The availability of modern, large format CCDs with smaller pixels enabled us to increase coverage of the spectrograph focal plane and an increase in the number of fibers. The total number of fibers for BOSS was increased to 1000 per plate, or 500 per spectrograph. This final number of fibers represents a practical limit given the desire to retain most of the original SDSS spectrograph optics while minimizing vignetting for objects imaged near the edge of the optics.

\subsubsection{Fiber Diameter}

As discussed in Section 2.1, the optimal fiber diameter is set by the desire to maximize $\mathrm{S} / \mathrm{N}$ for an extended source given the sky background. The BOSS galaxy sample extends to $z=0.7$, projecting a typical galaxy onto the telescope focal plane at an angular scale of a few arcseconds. The appropriate fiber size to maximize signal from the source while minimizing sky background for most distant galaxies is $2^{\prime \prime}$, corresponding to a fiber diameter of $120 \mu \mathrm{m}$. The BOSS fibers are therefore $2 / 3$ the original SDSS fiber diameter. The smaller diameter fiber scales with the reduction in pixel size $(24 \mu \mathrm{m}$ for SDSS and $15 \mu \mathrm{m}$ for BOSS) for the BOSS CCDs, thus preserving the desired spectral sampling of 3 pixels. The smaller diameter also facilitates the increase in the number of fibers described in the previous paragraph.

\subsubsection{Wavelength Range}

BOSS requires a modest increase in wavelength range compared with SDSS. The extension in the blue wavelength coverage is driven by the desire to measure absorption in the Ly $\alpha$ forest at $z>2.2$. The Ly $\alpha$ transition occurs at a wavelength of $1216 \AA$ in the quasar rest frame and the $\operatorname{Ly} \beta$ transition occurs at $1026 \AA$. The minimum wavelength requirement for BOSS was set at $3560 \AA$ so as to sample roughly half of the Ly $\alpha$ forest illuminated by a $z=2.2$ quasar between the $\operatorname{Ly} \alpha$ and $\operatorname{Ly} \beta$ transitions. The red cutoff was also extended from $9100 \AA$ to $10400 \AA$. The increase in coverage allows better coverage of the continuum redward of the $\mathrm{Ca} I \mathrm{~K}$ and $\mathrm{H}$ absorption lines that are the most common features used for redshift determination in the BOSS galaxy sample. The additional wavelength coverage enhances our ability to reliably classify passive galaxies up to a redshift of at least $z=0.8$. The BOSS wavelength coverage is larger than the original SDSS requirements by $1600 \AA$, enabled by increased detector size.

\subsubsection{Detector Read Noise and Dark Current}

To maintain Poisson-limited statistics in the $z=2.2$ region of the $\mathrm{Ly} \alpha$ forest, the BOSS blue channel detector must have a read noise less than $3.0 e^{-}$pixel $^{-1} \mathrm{rms}$. The requirement for the red channel is less stringent because the spectra are sky-dominated for most of the red channel wavelength range. The read noise for the red detector is required to be less than $5.0 e^{-}$pixel $^{-1} \mathrm{rms}$. To prevent significant noise contributions during an integration, a dark current that is less than $1.0 e^{-}$for the blue CCDs and less than $2.0 e^{-}$for the red CCDs during a 15 minute exposure is required. All of these requirements for detector performance were enabled by improvements in CCD technology.

\subsubsection{Resolving Power}

The resolution requirement for SDSS was broadly defined to balance the tradeoff between resolving absorption lines in relatively massive galaxies and maximizing wavelength coverage. The resolution requirement for BOSS is driven by the ability to fit the Balmer series in calibration stars between $3800 \AA<\lambda<4900 \AA$. From these fits we model surface gravity and stellar parameters and derive a synthetic spectrum for each standard star to calibrate spectrophotometry for the exposure.

Experience with SDSS indicates that a resolving power greater than $R=1400$ in this wavelength range is adequate for precise and reliable stellar classification. The BOSS galaxy spectra require resolution sufficient for achieving rms redshift error of $300 \mathrm{~km} \mathrm{~s}^{-1}$, achieved with a resolving power of $R>1000$ for the remainder of the wavelength range.

\subsubsection{Throughput and Signal-to-noise Ratio}

The required throughput is set by the desire to obtain spectra of 1.35 million luminous galaxies to a limiting magnitude of $i=19.9$ in five years. From observations of fainter galaxies in SDSS and a well-tested data reduction pipeline, it became clear that successful redshift classifications were possible with significantly lower $\mathrm{S} / \mathrm{N}$ than was achieved in SDSS. We established a rough goal of $\mathrm{S} / \mathrm{N}>3-4$ per $\AA$ for the faintest targets. With one hour exposures and 1000 fibers, BOSS requires a factor of two improvement in peak throughput. The improvement is possible through the use of state-of-the-art large format VPH gratings and modern CCDs with higher QE.

\subsection{Fiber System Upgrade}

A major part of the SDSS spectrograph upgrade for BOSS was the acquisition of nine new fiber cartridges with 1000 fibers to replace the original SDSS cartridges, which had 640 fibers. For BOSS, the cartridge design, including the castings themselves, is identical to that used for SDSS with the exception of revised slitheads to accommodate more fibers having a smaller diameter. The fiber mapper system follows the same philosophy as that used for SDSS, and fiber performance was tested in the same manner as the original SDSS fibers. For more detail on the cartridge design, the fiber mapper, and the method for assessing fiber performance see Section 2.2. Here we describe the BOSS fiber system upgrade, which for the most part is limited to the implementation of a larger number of smaller fibers.

\subsubsection{Fibers}

The optical fiber selected for BOSS is a step-index, all-silica, UV-enhanced fiber. It is similar to the fiber originally chosen for SDSS, except that it has a smaller core diameter of $120 \mu \mathrm{m}$. The core is surrounded by $25 \mu \mathrm{m}$ of a lower-index cladding with a cladding-to-core ratio of at least 1.4 , which confines the radiation by total internal reflection. The cladding is protected by a polyimide sheath, or buffer, bringing the outer diameter to $190 \mu \mathrm{m}$. The tolerance on the outer diameter was specified at $\pm 3 \mu \mathrm{m}$, with a maximum decentering of the core relative to the outer diameter of $6 \mu \mathrm{m}$. These tolerances were tighter than the $\pm 5 \mu \mathrm{m}$ outer diameter that was specified for BOSS prototype fibers with a larger outer diameter of $197 \mu \mathrm{m}$. The fiber was manufactured by Polymicro Technologies, Inc. and is known as FBP 120-170-190. The total length of each fiber is $1830 \pm 25 \mathrm{~mm}$.

The design of the fiber harnesses for BOSS follows that of the original SDSS fiber harnesses as described in Section 2.2, 


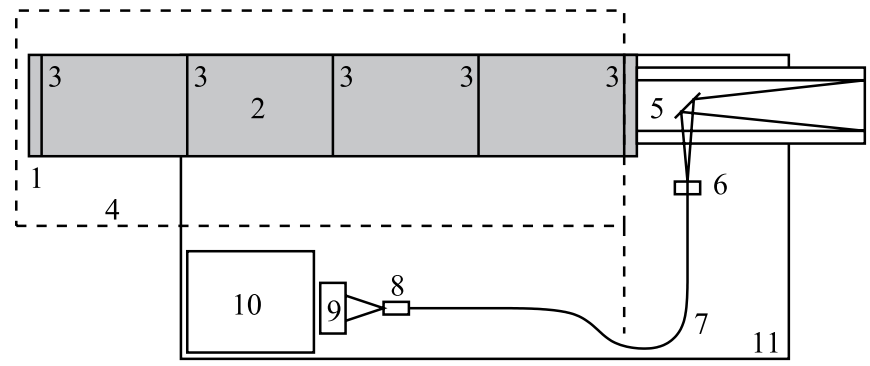

Figure 20. BOSS fiber FRD test setup diagram: (1) white LED array; (2) diffuser tube; (3) diffusing screens; (4) light shroud; (5) Newtonian reflector telescope stopped to $f / 5$ with $27 \%$ central areal obscuration; (6) fiber input bracket; (7) BOSS optical fiber; (8) v-groove block with fiber termination; (9) SBIG ST-8300M CCD camera; (10) data-acquisition computer; (11) optical breadboard $(24 \times 48$ in). System shown approximately to scale.

with the same overall dimensions and components. The same manufacturer, C-Technologies, Inc, was selected during a competitive bidding process. As in the original SDSS design, a fiber harness consists of 20 fibers, with one end mass-terminated by gluing the fibers into a metal v-groove block, while the free ends of the fibers are individually terminated in stainless steel ferrules. The v-groove blocks align the fibers on $260 \mu \mathrm{m}$ centers; the v-groove blocks are then glued to the slitheads. The v-groove blocks were machined in the Physics Machine Shop at the University of Washington. The stainless steel ferrules were manufactured by Swiss Screw Products. ${ }^{52}$ After the fiber was inserted into the ferrule and glued using a low-shrinkage adhesive, the end of the fiber was polished, removing a small amount of material and maintaining strict tolerances on planarity. The ferrule end was designed for insertion into the holes drilled in the plug-plates, where it is held by friction. As with the SDSS configuration, a jacket of nylon tubing on the ferrule end protects the fibers from breakage during repeated plugging and unplugging operations.

\subsubsection{Fiber Performance}

To measure the FRD characteristics of BOSS fibers as configured for science observations (as well as to characterize the effective exit pupil of the telescope-spectrograph system), the testing setup diagrammed in Figure 20 was constructed. This system was used to illuminate 18 fibers, one at a time, within a spare BOSS harness with a white-light input beam matched to the $f / 5$ SDSS $2.5 \mathrm{~m}$ telescope beam, including the $27 \%$ areal obscuration that removes the central rays from the cone of light incident on the telescope focal plane. The ferrule of each fiber under investigation was held in place using an aluminum plate drilled with a decommissioned SDSS-III drill bit. A tiptilt bracket was used to adjust the fibers for telecentricity with respect to the input beam. The diverging output beam was imaged using an SBIG ST-8300M CCD camera ${ }^{53}$ with $3326 \times 2504$ pixels of $5.4 \mu \mathrm{m}$ pitch, with the detector plane located a distance of $4.7 \pm 0.1 \mathrm{~cm}$ from the output termination of the fibers in the v-groove block. The resulting beam-profile images show a characteristic "fuzzy donut" of the telescope pupil convolved with the FRD within the fiber. The beam patterns were flux-centroided in the images, and the fractional flux enclosed as a function of distance from beam center was computed for each of the 18 fibers. The resulting curves are

\footnotetext{
52 Swiss Screw Products, Inc., http://www.swissscrew.com

53 SBIG Astronomical Instruments, http://www.sbig.com
}

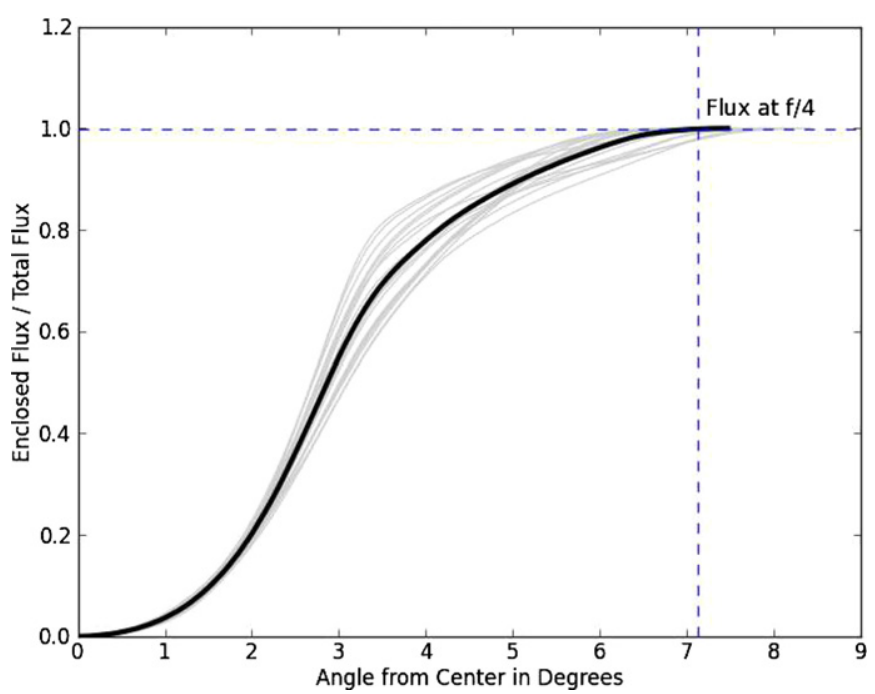

Figure 21. FRD curves for 18 tested BOSS fibers (thin gray), with average curve (thick black).

(A color version of this figure is available in the online journal.)

shown in Figure 21, with respect to an abscissa of beam halfangle in degrees. The mean curve for all 18 fibers is shown in thick black. We confirm that with an $f / 4$ spectrograph collimator, essentially all of the emergent flux is captured by the optical system.

\subsubsection{Slithead}

The BOSS slitheads are nearly identical in design to the original SDSS slitheads. They consist of a thin, stiff aluminum slitplate with a curved edge of radius $640 \mathrm{~mm}$. There are two slitheads per cartridge, each having 500 fibers. The $120 \mu \mathrm{m}$ diameter fibers are mounted in groups of 20 in v-groove blocks, with $25 \mathrm{v}$-groove blocks being glued to each slithead. The centerto-center spacing between fibers on adjacent v-groove blocks is $624 \mu \mathrm{m}$, compared with $260 \mu \mathrm{m}$ between fibers within a $\mathrm{v}$-groove block. The total length of the arc is $138.6 \mathrm{~mm}$ from outside edge to outside edge of the first and last fibers, slightly taller than the original SDSS design.

\subsection{Optical Upgrades}

\subsubsection{Optical Design Overview}

The optical design of the BOSS spectrograph is nearly identical to the original SDSS design. For BOSS the bandpass is extended in both channels to cover $3560 \AA<\lambda<10400 \AA$. Additionally the replicated surface relief gratings used in the SDSS design were replaced with VPH gratings, and modern CCDs with higher QE and smaller pixels were installed; the combined effect being an impressive $70 \%$ peak instrumental efficiency, a significant improvement over the $45 \%$ peak of the SDSS design.

Figure 22 shows the BOSS optical layout. Light enters the spectrograph through $120 \mu \mathrm{m}$ diameter fibers, which terminate at a curved slithead in the same manner as the original SDSS slithead. The collimator, unchanged from the original design, collimates the $f / 4$ beam producing a $160 \mathrm{~mm}$ diameter beam, reflecting it back toward the dichroic beamsplitter. Light blueward of $640 \mathrm{~nm}$ is reflected into the blue channel, with the remaining light being transmitted into the red channel. A VPH grism in each channel disperses the light, which is then imaged by upgraded cameras, each containing a single $4 \mathrm{k} \times 4 \mathrm{k}$ CCD 


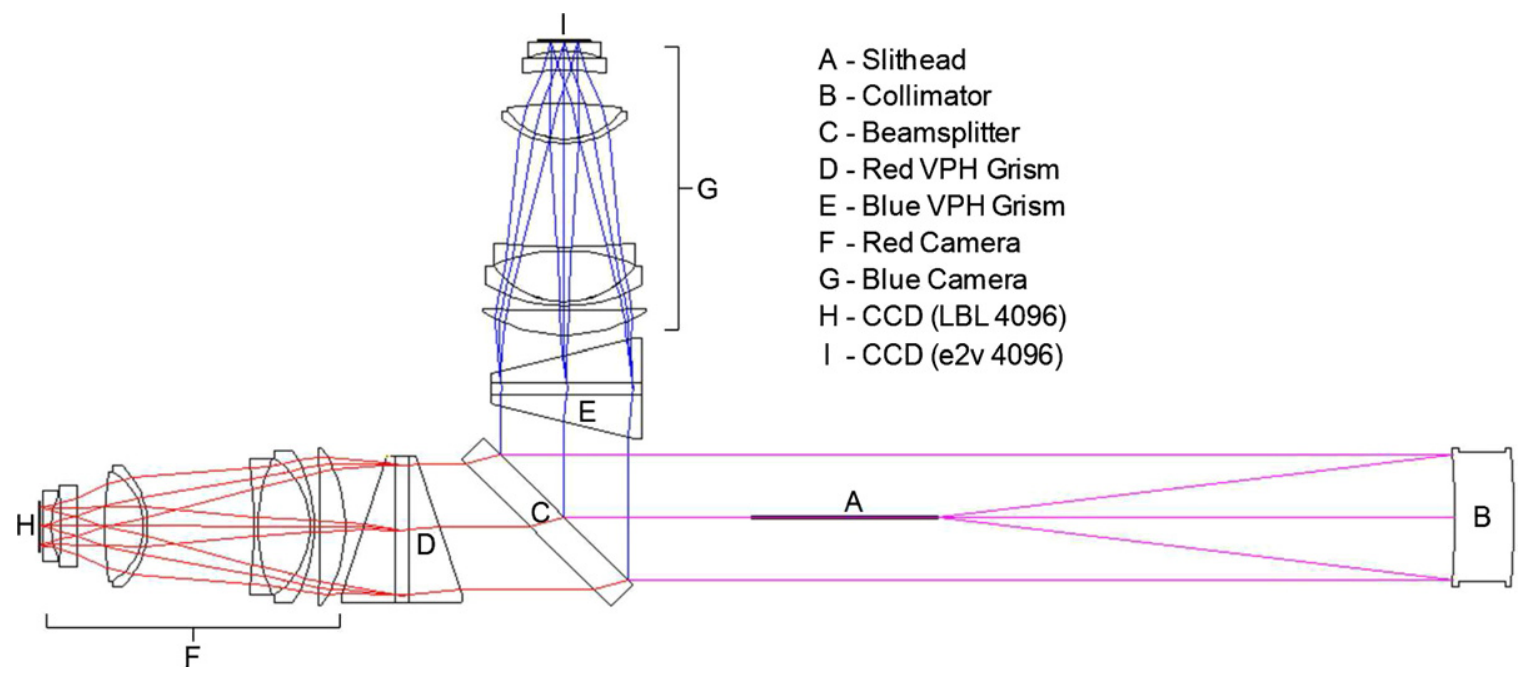

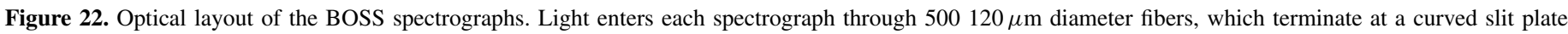

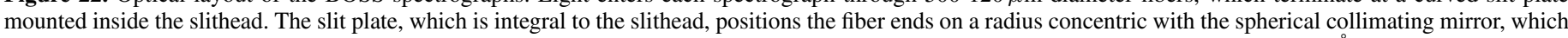

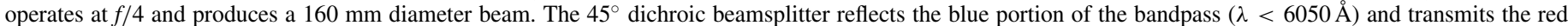

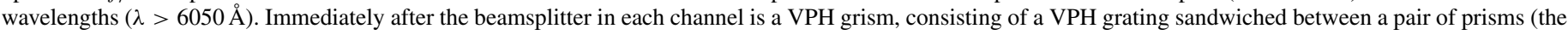

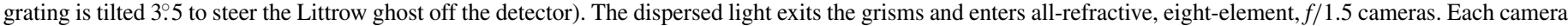

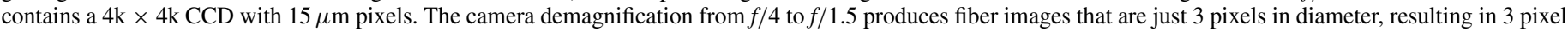
tall spectra on the detector.

with $15 \mu \mathrm{m}$ pixels. The use of smaller fibers and correspondingly smaller pixels, along with the same collimator/camera demagnification preserves the scale of the spectra on the detector, thus yielding 3 pixel wide spectral profiles separated by 6 pixels center-to-center, identical to the SDSS configuration.

\subsubsection{Collimator}

The BOSS survey started in 2009 with the same collimators used for SDSS. At that time protected silver coatings offering significantly higher reflectivity at the blue end of the spectrum had been realized on small optics. Given the obvious benefit of such a coating, the BOSS project set out to re-coat the collimators, beginning with the spare collimator originally produced for SDSS. However, realization of such a coating on a large surface such as the collimator was challenging due to the process control required to achieve very uniform layer depositions over a large surface area. Attempts by one vendor ultimately failed after more than a year of trial and error. A successful coating was achieved by Infinite Optics ${ }^{54}$ in late 2011, and newly coated collimators have been installed in both spectrographs.

\subsubsection{Beamsplitter}

The dichroic beamsplitter in each spectrograph was replaced for BOSS given the expanded bandpass of the instrument. For BOSS, wavelengths shorter than $605 \mathrm{~nm}$ are reflected by the new coating, a $15 \mathrm{~nm}$ shift toward the red as compared with SDSS. Although the BOSS bandpass is extended much more into the red than the blue, the crossover wavelength was shifted only a small amount. In the process the entire beamsplitter, i.e., substrate and coating, were replaced, a logical consequence of the desire to keep the instrument operational while the new coatings were applied, and to mitigate the risk of potentially taking the instrument offline for an extended period should stripping the original coating lead to damage of the substrate.

\footnotetext{
54 Infinite Optics, http://www.infiniteoptics.com
}

An additional, and significant, consideration in replacing the beamsplitters was the desire to obtain a denser coating using ion-assisted deposition, which would be more immune to wavelength shifts with variations in humidity. The SDSS beamsplitters suffered from throughput variations with changes in humidity inside the instrument, which occurs due to the periodic insertion of new cartridges. Each time a cartridge is installed the slithead door is opened, allowing moist air to displace the dry air injected into the bench to protect the calcium fluoride singlet in the camera. Once the door closes the humidity level slowly reduces back to its original near-zero level. The effect on the data are throughput variations as large as $5 \%$ in $\sim 50 \AA$ wide ripples in the $3800-4800 \AA$ range, and shifts of the dichroic transition region. A more detailed description of this problem and its removal from the data can be found in Section 8.1 of Abazajian et al. (2009). There is no evidence of throughput variations with the new coating.

\subsubsection{Gratings}

As in the SDSS spectrographs, a grism immediately follows the beamsplitter in each channel. However, the significant increase in efficiency offered by VPH gratings convinced us to abandon the SDSS replicated surface relief grism design in favor of a VPH grism. The grism consists of a VPH transmission grating sandwiched between two prisms; the use of prisms is necessary to preserve the straight-through beam layout of the SDSS design.

The BOSS design utilizes a ruling density of $4001 \mathrm{~mm}^{-1}$ in the red channel and $5201 \mathrm{~mm}^{-1}$ in the blue channel. The grisms were designed independently at Johns Hopkins University (using the Zemax raytrace program) and at Kaiser Optical Systems, Inc. ${ }^{55}$ (KOSI), who built the gratings. Both the VPH grating substrates and the prisms are made from BK7 or equivalent glass. The blue channel components were made from Ohara Corporation's BSL7Y glass, a BK7 equivalent with enhanced UV transmission. The prism apex angles (total for

\footnotetext{
55 Kaiser Optical Systems, Inc., http://www.kosi.com
} 
the two-prism assembly) are $28^{\circ}$ and 35.6 for the blue and red grisms, respectively.

A phenomenon now well-known to builders of VPH gratingbased spectrographs, is that of the Littrow ghost, or recombination ghost as it is sometimes called (Burgh et al. 2007). This is an undispersed (white-light) ghost image of the entrance slit or fibers of the instrument, and appears in the image plane at the location of the blaze or Bragg wavelength. Because the ghost is undispersed, its intensity can be quite bright relative to the nearby features of interest in the spectrum. This particular type of ghost is present in any spectrograph operating in the Littrow configuration $(\alpha=\beta)$ but plagues in particular instruments with VPH gratings as they are so often used at or near the Littrow mode in order to obtain the highest efficiency possible.

To better understand the implications of the recombination ghost for the BOSS spectrographs, we undertook a rather extensive raytrace analysis of the possible ghost paths initiated by a reflection off the detector. In addition, we analyzed the paths of the zero-order light and the undesired diffracted orders with any appreciable efficiency predicted by KOSI's rigorous coupled-wave analysis of the grating designs. In order to move the recombination ghost away from the useful part of the spectrum, the grating must be moved away from the Littrow configuration. One way to do this with a VPH grating is by rotating the fringes within the gelatin layer during exposure to be off-normal with respect to the plane of the grating itself. The grating is rotated by the same angle in use such that the fringe orientation is returned to the Bragg condition where efficiency is highest, but the grating is no longer at Littrow. Our analysis indicated a rotation of $3^{\circ}$ would be sufficient to move the ghost completely off the detector, and we settled on 3.5 to provide a bit of margin. To accommodate the tilted grating, each prism deviates from a right angle by the same 3.5 tilt, with one prism having an apex angle that is 3.5 larger and the other 3.5 smaller than the right angle design of an untilted grating. We chose this approach, rather than using right angle prisms and rotating the entire grism assembly as a whole, due to extreme space constraints within the upgraded central optics assembly. Figure 23 shows the nominal, first-order light path in the upper panel, and in the lower panel the recombination ghost path arising within the grating layer.

For BOSS, the VPH red grating peak efficiency is $82 \%$ as compared with $64 \%$ for SDSS, and the blue grating peak efficiency is $80 \%$ as compared with $50 \%$ for SDSS.

\subsubsection{Cameras}

Both red and blue camera optical designs were reoptimized for BOSS. The revised designs use the original SDSS prescriptions for the first six elements with the same multiplet configuration: a singlet, followed by a contact triplet, and then a contact doublet. As in the original design this sequence of elements is followed by two field flatteners, which reside just in front of the detector. For BOSS the spacings between these groupings were modified slightly, and the field flatteners were completely redesigned for improved imaging performance and larger focal plane arrays. These changes were motivated in part by the expanded wavelength range in each channel, and in part by new, larger $(4 \mathrm{k} \times 4 \mathrm{k}$ as compared with $2 \mathrm{k} \times 2 \mathrm{k})$, detectors having smaller pixels ( $15 \mu \mathrm{m}$ for BOSS as compared with $24 \mu \mathrm{m}$ for the SDSS design) and working over a slightly larger field. Hence, imaging performance requirements for BOSS are more stringent than for SDSS. Nonetheless, the BOSS design uses the same basic eight-element configuration as the original SDSS

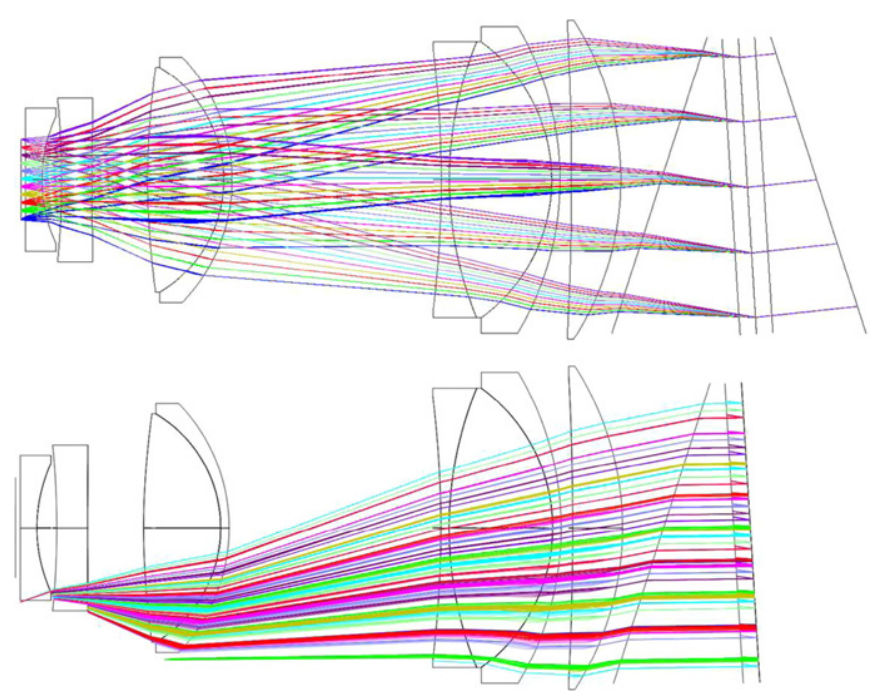

Figure 23. Raytrace layouts illustrating the light path for the recombination (Littrow) ghost. In these layouts the CCDs are on the left, the VPH grism assembly (or part thereof) is on the right. The desired, first-order diffracted light (top) travels right to left through the grism and camera lenses to the CCD. The recombination ghost arising within the grating layer (bottom) is initiated by a reflection off the $\mathrm{CCD}$, which sends some small percentage of the light back through the camera in a left-to-right direction. The camera recollimates this light, which then encounters the grating for a second time. Some of this light is reflected and recombined by the grating and travels back through the camera to the CCD. It is this last, right-to-left pass that is shown in the layout, the other passes are omitted for clarity. Note how the grating is rotated by 3.5 with the prism assembly in order to direct the recombination ghost entirely off the CCD.

cameras. In fact, the singlet and doublet elements are re-used from SDSS. For the triplet, new lenses were fabricated for the first and third elements to take advantage of improved coatings that are properly matched to the BOSS bandpasses in each channel.; the central $\mathrm{CaF}_{2}$ triplet, which is not coated, was re-used from SDSS. For the field flatteners, new lenses were fabricated.

\subsubsection{Optical Prescription}

Table 2 shows the optical prescription for the BOSS spectrograph. The surface descriptions are the same as in Table 1. As with the SDSS spectrographs, all materials are from Ohara Glass except for $\mathrm{CaF}_{2}$ and the lens couplant, Dow-Corning Q2-3067.

\subsubsection{Predicted Optical Performance}

The following sections discuss the predicted optical performance of the BOSS spectrographs, including image quality, spectral resolution, and throughput. Measured performance is discussed in Section 4.

Image quality. Spot diagrams for the red and blue channels are shown in Figures 24 and 25, respectively. The spots are shown within a $45 \mu \mathrm{m}$ diameter circle, representing the diameter $\left(2^{\prime \prime}\right)$ of the imaged BOSS fiber on the detector. Each diagram covers the full respective bandpass of the channel, and field points cover the full length of the slithead. The average rms spot diameter for the red channel is $18.3 \mu \mathrm{m}$ and the maximum rms diameter is $27.5 \mu \mathrm{m}$. For the blue channel the average rms diameter is $19.6 \mu \mathrm{m}$ and the maximum rms diameter is $30.9 \mu \mathrm{m}$. Compared with the SDSS image quality, the BOSS design is slightly better on average, and this is over an expanded bandpass and larger field of view (taller slit). However, the fiber diameter is smaller for BOSS as are the detector pixels. Nonetheless, when the geometric aberrations are convolved with the fiber diameter the resulting FWHM is still very near $45 \mu \mathrm{m}$, which matches three rows of pixels on the detector. 
Table 2

Optical Prescription for the BOSS Spectrographs

\begin{tabular}{|c|c|c|c|c|c|c|}
\hline \multirow[t]{2}{*}{ Surface } & \multicolumn{2}{|c|}{ Radius (mm) } & \multicolumn{2}{|c|}{ Thickness (mm) } & \multicolumn{2}{|c|}{ Material } \\
\hline & Blue & Red & Blue & Red & Blue & Red \\
\hline Slithead & -640.080 & -640.080 & 630.123 & 630.123 & Air & Air \\
\hline Collimator mirror & -1263.904 & -1263.904 & -1087.831 & -1087.831 & Air & Air \\
\hline Prism & $\operatorname{Plano}(-14.0)$ & Plano $(-17.8)$ & -33.604 & -37.414 & BSL7Y & BK7 \\
\hline Cover plate & Plano(-3.5) & Plano(-3.5) & -8.000 & -8.000 & BSL7Y & BK7 \\
\hline Grating & Plano $(-3.5)$ & Plano $(-3.5)$ & -8.000 & -8.000 & BSL7Y & BK7 \\
\hline Prism & Plano(14.0) & Plano(17:8) & -33.376 & -40.005 & BSL7Y & BK7 \\
\hline Singlet,front & -182.804 & -182.804 & -30.531 & -30.531 & $\mathrm{CaF}_{2}$ & $\mathrm{CaF}_{2}$ \\
\hline Singlet,back & -1813.560 & -1813.560 & -6.604 & -6.960 & Air & Air \\
\hline Triplet,first,front & -185.522 & -185.522 & -5.156 & -5.156 & LAL7 & LAL7 \\
\hline Triplet,first,back & -105.867 & -105.867 & -0.076 & -0.076 & Q2-3067 & Q2-3067 \\
\hline Triplet,second,front & -105.867 & -105.867 & -63.525 & -63.525 & $\mathrm{CaF}_{2}$ & $\mathrm{CaF}_{2}$ \\
\hline Triplet,second,back & 226.136 & 226.136 & -0.076 & -0.076 & Q2-3067 & Q2-3067 \\
\hline Triplet,third,front & 226.136 & 226.136 & -5.105 & -5.105 & BSM2 & BSM2 \\
\hline Triplet,third,back & -661.416 & -661.416 & -131.750 & -129.718 & Air & Air \\
\hline Doublet,first,front & -110.490 & -109.423 & -5.080 & -5.080 & BAL35Y & BAL35Y \\
\hline Doublet,first,back & -87.274 & -81.331 & -0.076 & -0.076 & Q2-3067 & Q2-3067 \\
\hline Doublet,second,front & -87.274 & -81.331 & -44.120 & -46.990 & FPL51Y & FPL51Y \\
\hline Doublet,second,back & $429.006^{\mathrm{a}}$ & $432.892^{\mathrm{b}}$ & -44.094 & -34.823 & Air & Air \\
\hline Flattener,first,front & 294.716 & $3362.350^{c}$ & -14.961 & -18.796 & BSM51Y & $\mathrm{CaF}_{2}$ \\
\hline Flattener,first,back & Plano & -503.453 & -9.398 & -12.141 & Air & Air \\
\hline Flattener,second,front & 99.187 & 94.971 & -6.096 & -10.185 & BSM51Y & S-LAH59 \\
\hline Flattener,second,back & Plano & Plano & -3.175 & -3.048 & Air & Air \\
\hline $\mathrm{CCD}$ & Plano & Plano & 0 & 0 & Silicon & Silicon \\
\hline
\end{tabular}

Notes.

${ }^{a}$ Aspheric surface coefficients: $\alpha_{4}=7.517856 \mathrm{E}-04, \alpha_{6}=-5.900703 \mathrm{E}-05, \alpha_{8}=2.644114 \mathrm{E}-06$.

${ }^{\mathrm{b}}$ Aspheric surface coefficients: $\alpha_{4}=-7.880071 \mathrm{E}-04, \alpha_{6}=6.800388 \mathrm{E}-05, \alpha_{8}=-2.676036 \mathrm{E}-06$.

${ }^{\mathrm{c}}$ Aspheric surface coefficients: $\alpha_{4}=6.490621 \mathrm{E}-05, \alpha_{6}=5.825222 \mathrm{E}-05, \alpha_{8}=-4.033623 \mathrm{E}-05$.

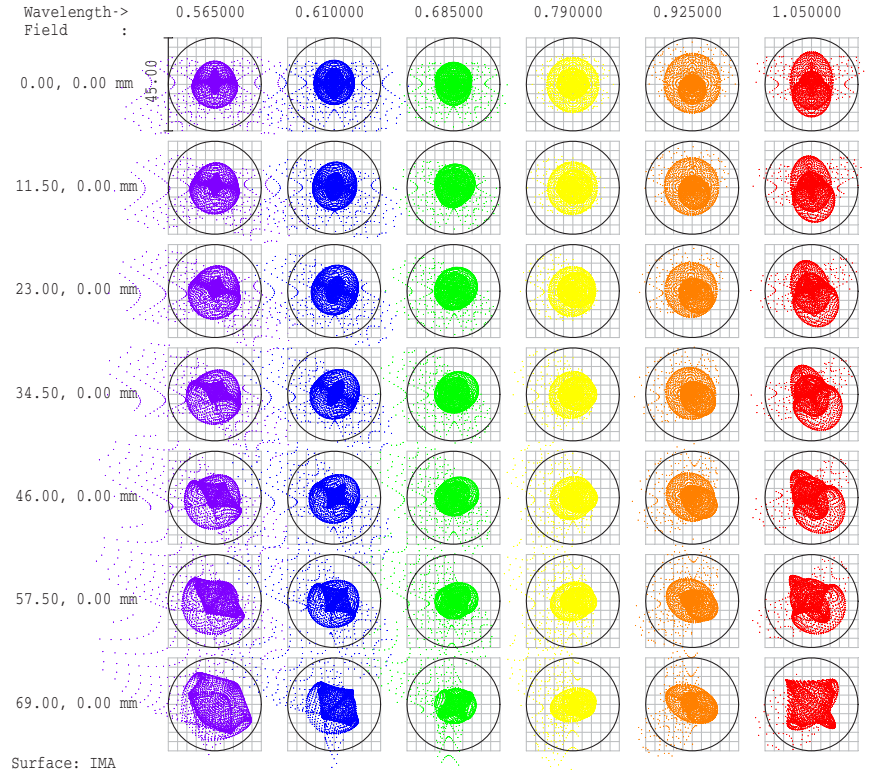

Figure 24. Spot diagram for the BOSS red channel. The average rms spot diameter is $18.3 \mu \mathrm{m}$ and the maximum rms diameter is $27.5 \mu \mathrm{m}$.

Spectral resolving power. Spectral resolving power predictions for the BOSS spectrograph are shown in Figure 26. These predictions were derived in the same manner as for the SDSS spectrograph resolving power discussed in Section 2.3. Comparing the BOSS and SDSS predictions, the blue channel is slightly lower than the SDSS design, while in the red channel the resolving power is slightly higher. The improved resolving power in

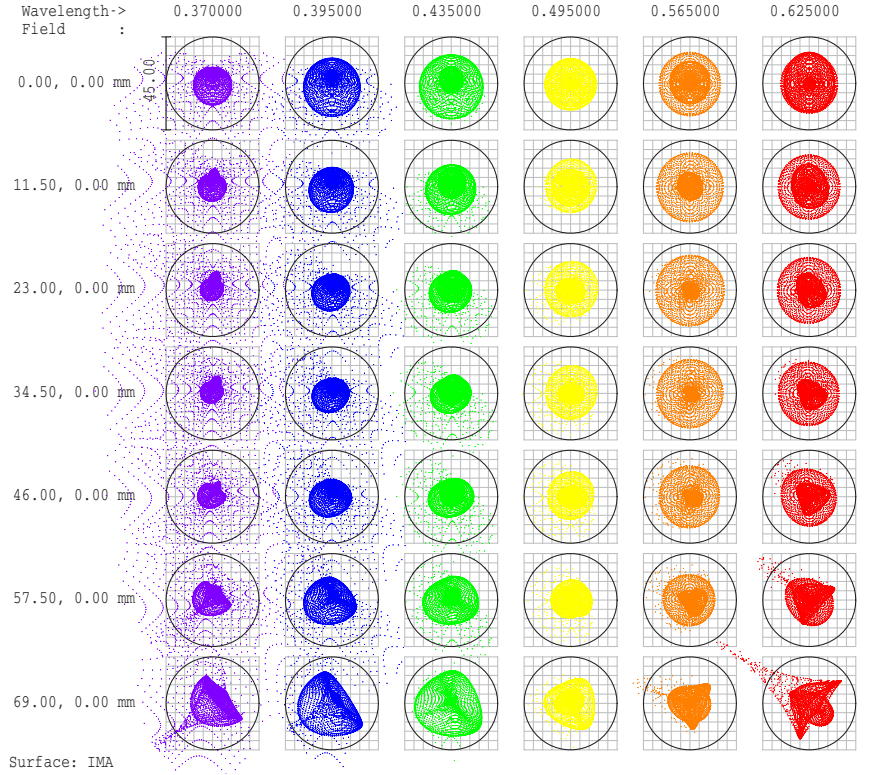

Figure 25. Spot diagram for the BOSS blue channel. The average rms diameter is $19.6 \mu \mathrm{m}$ and the maximum rms diameter is $30.9 \mu \mathrm{m}$.

the red channel is the result of a larger detector with more pixels, more than offsetting the expanded wavelength coverage. In the blue channel, the grating dispersion was deliberately chosen to underfill the detector in the spectral direction, thus maximizing the $\mathrm{S} / \mathrm{N}$ while providing sufficient resolving power to carry out the desired science.

We also present a two-dimensional contour image, Figure 27, of the BOSS CCDs, showing the rms width $\sigma_{p}$ of the line-spread 


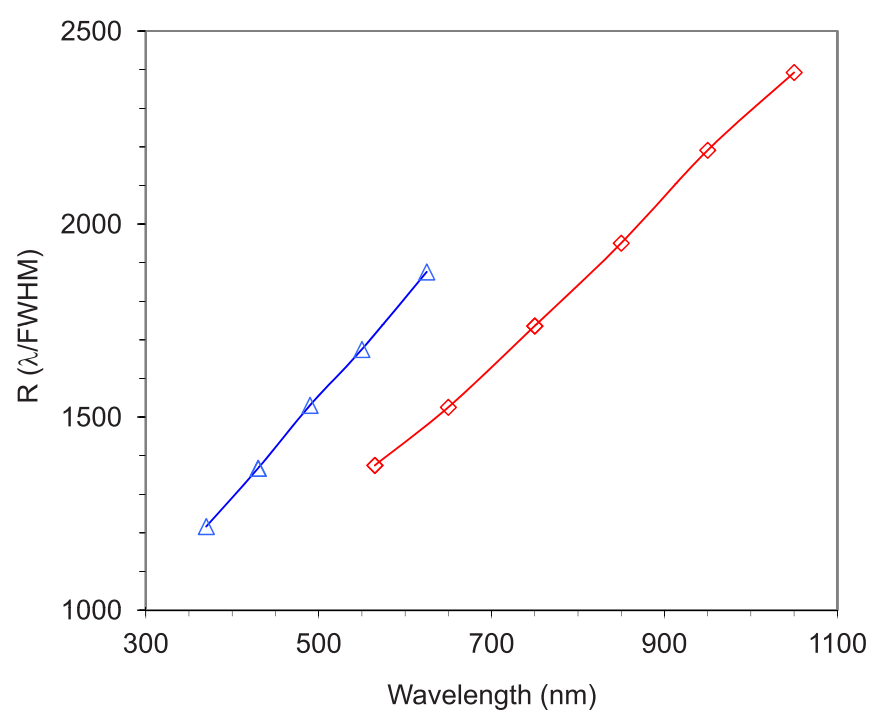

Figure 26. Predicted resolving power, $R$, as a function of wavelength for the BOSS spectrographs. As with the SDSS design, predictions are derived from the FWHM of simulated, uncollapsed images computed using Zemax. In reality, the resolving power of the instrument is determined using collapsed spectra, so the prediction here is on the low side. However, the Zemax analysis assumes perfect optics and alignment. For this reason the predictions have been based on uncollapsed spectra, which is the conservative approach. The expectation is that the actual resolving power will be somewhat better than shown here.

function in native pixels to demonstrate the predicted variation in resolution over the focal plane. The FWHM $=2.35 \times \sigma_{p}$. Note that the linewidths are moderately degraded at the shortest wavelengths of the blue channel near the ends of the slit.

Throughput. As was the case for the SDSS spectrographs, the total throughput, on-sky, for the BOSS spectrographs was predicted from an end-to-end component model as a function of wavelength. Included in the model were: atmospheric extinction, seeing (slit) losses, telescope, fibers, collimator, dichroic, grism, camera, and CCD QE. Atmospheric extinction was modeled at one airmass as before. Seeing losses were modeled using a double Gaussian PSF with a FWHM of $1^{\prime \prime}$ centered in a $2^{\prime \prime}$ aperture, resulting in a $17.5 \%$ throughput loss assumed flat across the bandpass. The telescope efficiency is based on measured data for $\mathrm{CO}_{2}$-cleaned bare aluminum mirrors (Wilson, RTO II), along with simulated anti-reflection coating curves to match the specifications of the two wide field corrector lenses (a small overall effect). Measured curves were used for the dichroic, grism, camera coatings, and CCD QE. The manufacturer's curve for Denton FSS99 silver was used for the collimator coating. The new silvered coating applied to the collimators in 2012 by Infinite Optics, led to a system throughput somewhat better than what is described in this paper, particularly at short wavelengths. Internal transmission curves for the camera glasses were obtained from the manufacturer's data sheets. The fiber efficiency (85\%) is based on lab measurements, representing an average value for the fibers measured and assumed flat across the bandpass. An additional 3.6\% loss is included for FRD beyond the $f / 4$ beam the collimator was designed to accept. Figure 28 shows the individual component efficiencies used for this model along with the total expected system throughput. Peak efficiencies in the blue and red channels are similar at about $31 \%$. This represents a major gain relative to the SDSS spectrographs, where a similar component model resulted in expected peak efficiencies of $17 \%$ and $22 \%$ for the blue and red channels, respectively.
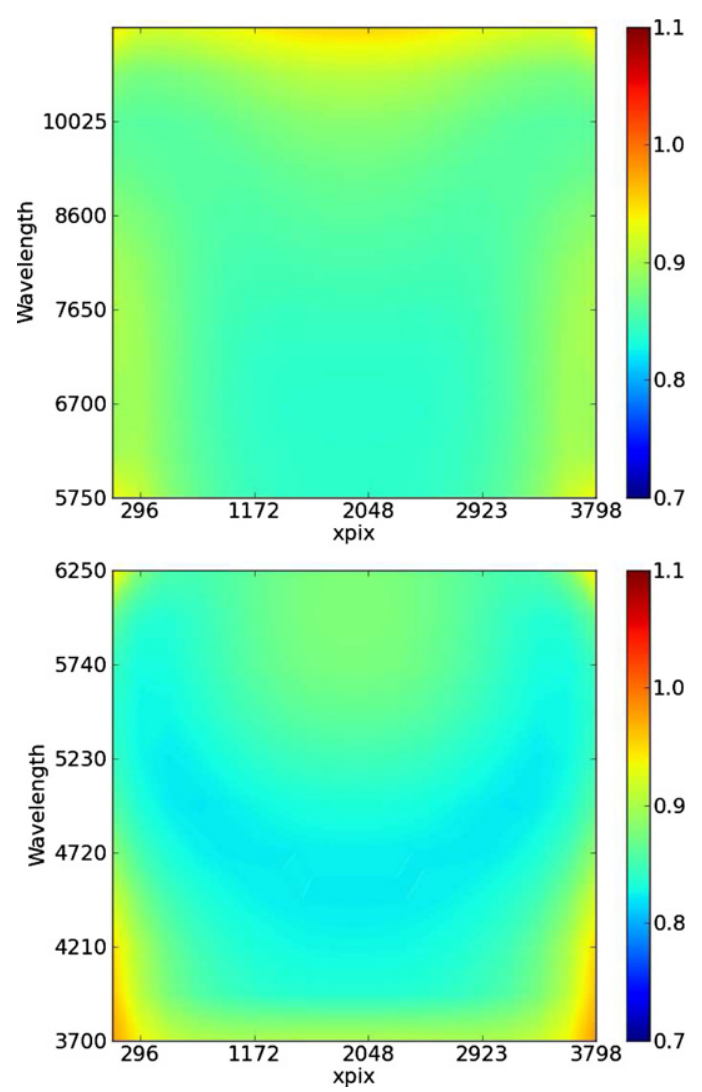

Figure 27. Contour image of the BOSS CCDs showing the rms width $\sigma_{p}$ of the line-spread function in native pixels to demonstrate the predicted variation in resolution over the focal plane. The FWHM $=2.35 \times \sigma_{p}$; red CCD (top) and the blue CCD (bottom). The wavelength decreases from top to bottom.

\subsection{Mechanical Upgrades}

\subsubsection{Overview}

Figure 29 shows the mechanical layout for the BOSS spectrograph. The revised design is nearly identical to that for SDSS. In fact, most of the hardware in the SDSS spectrograph is reused for BOSS. Upgrades include new fiber cartridges and slitheads, a new central optics assembly to house new beamsplitters and VPH grisms, modified lens mounts and hardware to accommodate the revised optical design, and redesigned dewars for the new CCDs. The fiber system upgrades were discussed in Section 3.2. In this section we describe the details of the remaining opto-mechanical upgrades.

\subsubsection{Collimator}

The collimator opto-mechanics are the same for BOSS as they were for SDSS. However, a subtle, yet important, modification was made by inserting a small mask in front of the collimator. A $3 / 16$ inch wide vertical strip was placed down the middle of the collimator, aligned parallel to the slithead, to prevent reflections off the tips of the fibers and back into the optical system.

\subsubsection{Central Optics Assembly}

As described in Section 3.3, the right angle grisms used in SDSS were replaced with VPH grisms for BOSS. The new grisms consist of a plane, parallel, VPH grating sandwiched between two prisms. Given the new grism form factor, the central optics assembly was redesigned. The revised configuration can be seen in Figure 29. The new design utilizes the same basic 


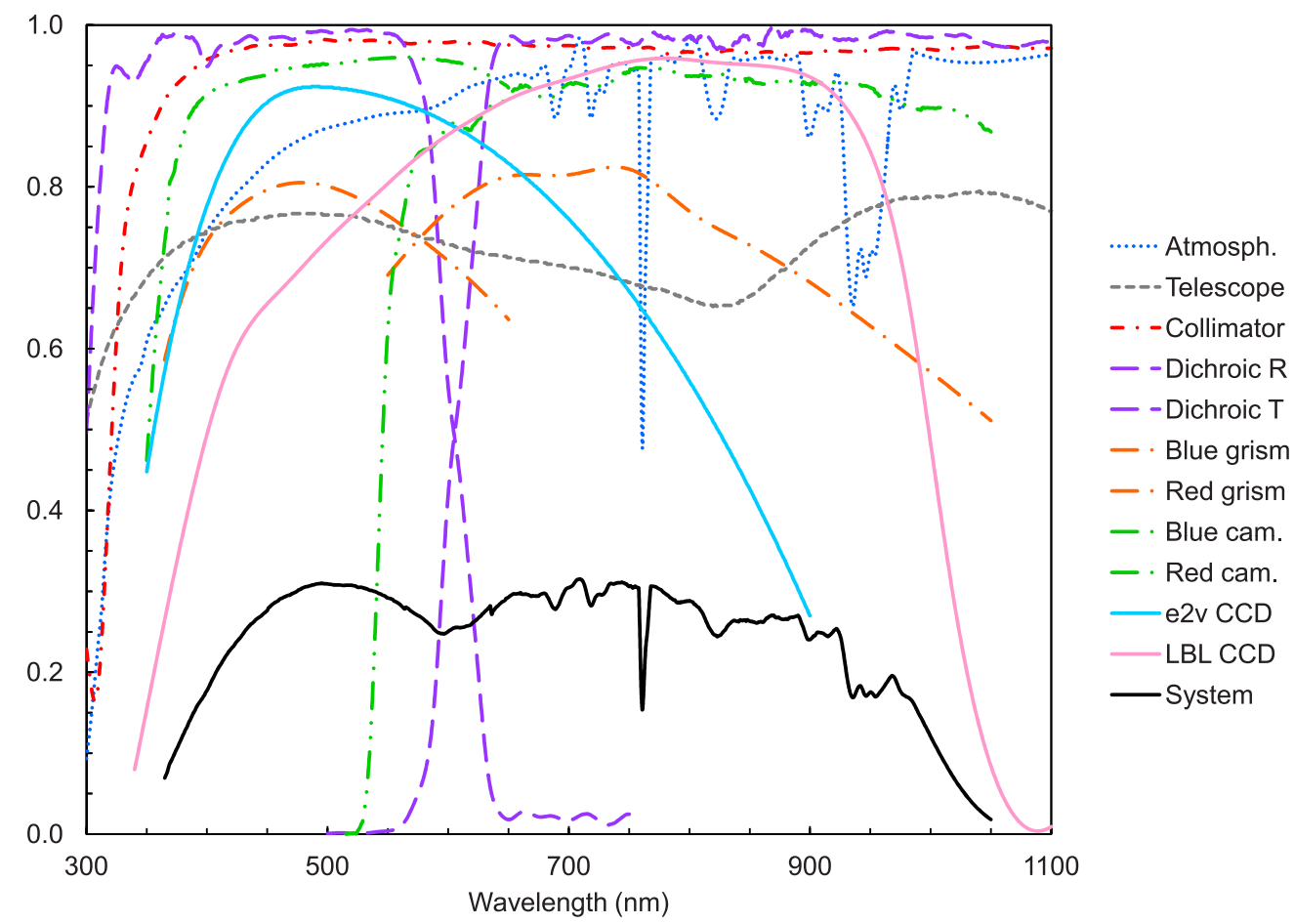

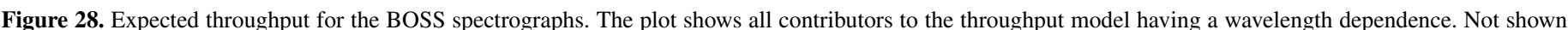

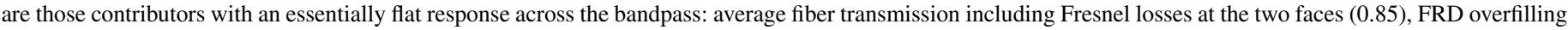

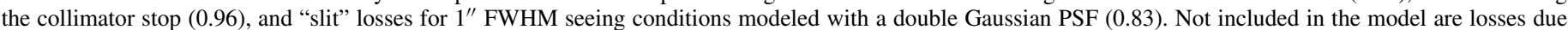

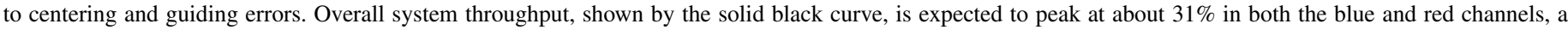
significant gain over the original SDSS spectrographs.

mounting schemes as its predecessor. Each grism is kinematically located by six reference points and registered against those points using spring plungers. Retention of the dichroic is identical to the SDSS design with simple spring plungers to preload the optic against six reference points. As in the original design, all but 1 of the 18 locating references ( 6 for each of the 3 optics) are machined into the base of the assembly, which ensures accurate placement. A mask was added in this design just in front of each grism to prevent undispersed light from sneaking past the grism, something that was discovered during commissioning. Overall the assembly is slightly larger and more massive than the SDSS design. The mass of the BOSS version is $44 \mathrm{~kg}$ as compared with $39 \mathrm{~kg}$ for the SDSS configuration. Fortunately, the opening in the side of the optical bench was large enough to accept the larger-sized assembly. However, a minor adjustment to the guide rails inside the bench was required to allow for a slight shift in the location of the assembly relative to the opening in the bench.

\subsubsection{Camera Opto-mechanics}

Exclusive of the dewars, which are discussed below, only minor opto-mechanical adjustments to the cameras were required to accommodate the BOSS optical design. The BOSS cameras (both red and blue) have new field-flattening lenses and revised singlet-to-triplet and triplet-to-doublet spacings. To achieve the increased spacing between the singlet and the triplet the singlet cells were remade, the new cells being thicker than the original. The triplet-to-doublet spacings were adjusted by fabricating new inner barrels with the appropriate length. Figure 30 shows a cross-section of the BOSS blue camera.

While the SDSS cameras performed well for many years, the grease coupling layers in the triplet degraded slowly over time, the result being a random web-like pattern of voids, which reduced transmission and increased scattered light. Considerable effort was invested in resolving this problem. Tests using the grease originally used, Dow Corning Q2-3067, showed that these voids begin to appear after just one thermal cycle. Hence, re-coupling the lenses with fresh grease as a routine maintenance effort was not a viable solution. After much trial and error using alternative optical couplants such as Sylgard 184, ${ }^{56}$ and an optical gel, Lightspan LS6943 from NuSil, ${ }^{57}$ both of which failed during thermal testing, it was determined that optical coupling fluid (i.e., an optically transparent oil) was the only reliable solution.

The triplet cell was redesigned with the lenses being coupled by Cargille Laser Liquid $1074 .{ }^{58}$ The revised design is shown in Figure 31. In the new design, the $0.25 \mathrm{~mm}$ gap between the lenses is set by a kapton shim with fingers that extend radially inward near the lens outer diameters. The space between the fingers allows the laser liquid to penetrate into the gap. Silicone O-rings seal the liquid volume at the rear face of the last element and at the rear face of the front element. Two reservoirs with pistons were integrated as well to accommodate differences in volumetric expansion between the laser liquid and the space it occupies. A drain and fill port, $180^{\circ}$ opposed, penetrate the outer diameter of the cell to facilitate filling and draining the liquid.

Some consideration was given to the choice of O-ring material given that the Robert Stobie Spectrograph (RSS; Kobulnicky et al. 2003) suffered UV transmission loss when the coupling fluid became contaminated from interaction with the polyurethane material used for the expansion bladder in their

\footnotetext{
56 Produced by Dow Corning, http://www.dowcorning.com

57 NuSil Technology, http://www.nusil.com

58 Cargille Labs, http://www.cargille.com
} 


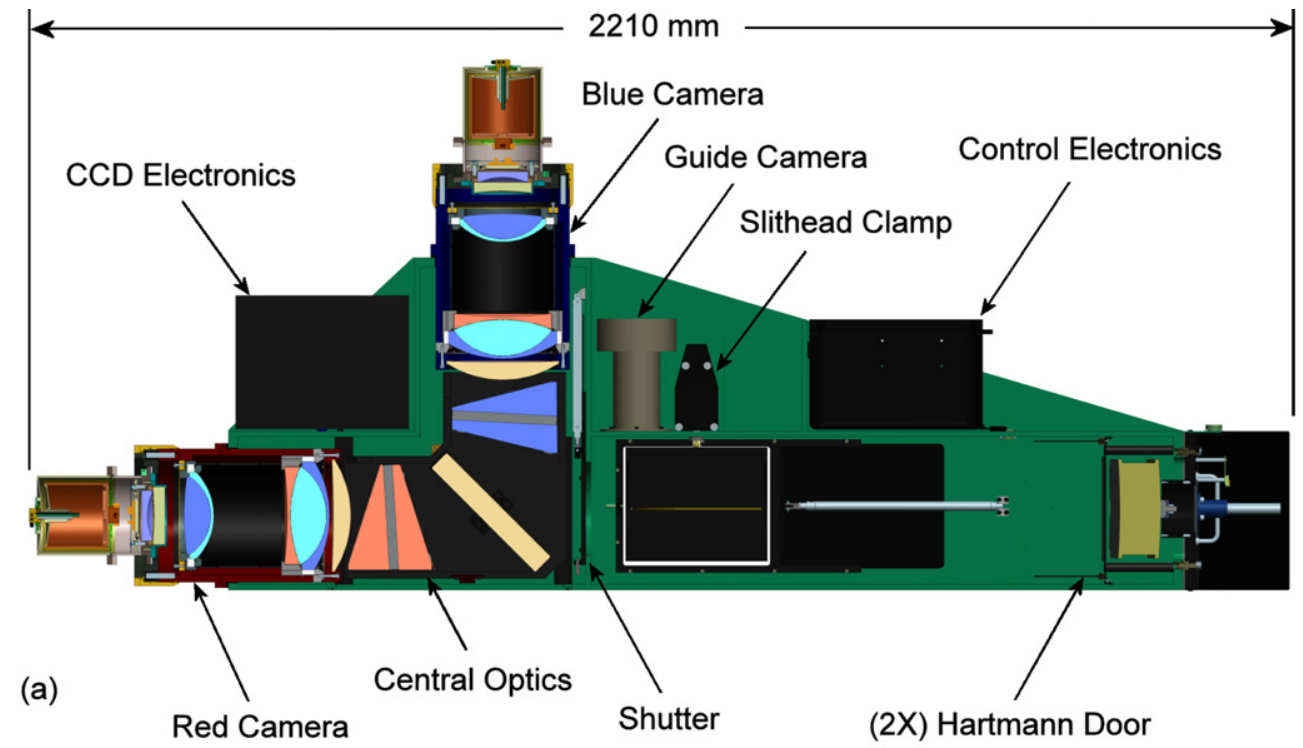

(3X) Spectrograph Mount

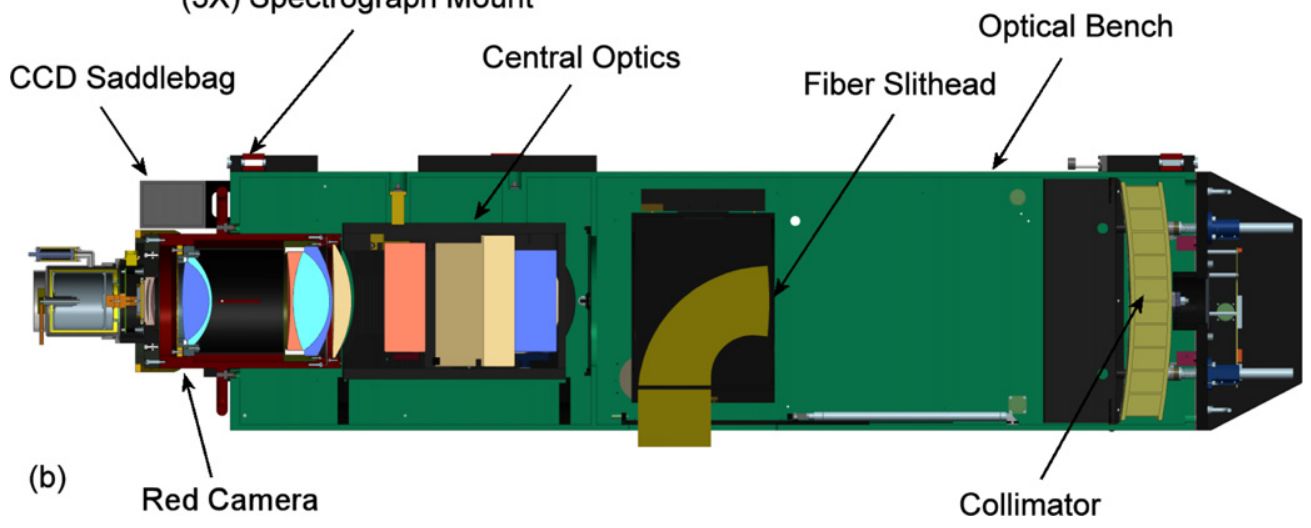

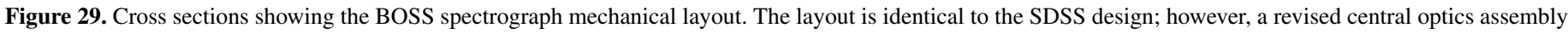

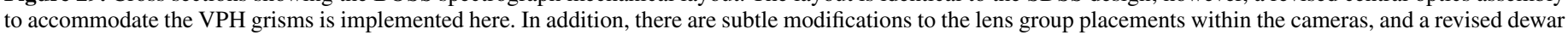
design to accommodate new CCDs.

liquid-coupled cell. At the time the BOSS triplet cell was designed, we were unable to conduct our own material compatibility testing, other than simple qualitative tests; the schedule did not permit it. Instead, we relied on data obtained from studies done for the Binospec instrument (D. Fabricant 2010, private communication), and from discussions with Cargille Labs. Based on both sources of data, silicone was chosen for the large O-rings that seal against the front and rear elements, and Viton was used to seal the pistons and drain plugs. Silicone was used for the main seals because it was available in a lower durometer (i.e., a softer material). Data published in 2010 on the RSS experience (Nordsieck et al. 2010) confirmed that both silicone and Viton have acceptably low reactivity with Laser Liquid, however, in general, silicone appears to be better for wavelengths $<380 \mathrm{~nm}$.

In addition to the changes made to accommodate the revised optical design, the focus locking mechanism was also modified. It had been determined that while the locking mechanism was sufficient to lock the dewar to the focus ring, the focus ring itself was not properly locked to the barrel. As a result, the blue camera in Spectrograph 1 would suddenly drift out of focus within a predictable, narrow range of motion on the rotator; see Section 4.3 for a more detailed discussion of this issue. To solve the problem, three large locking screws were installed in the dewar flange, which press against the back face of the barrel. Tightening the screws preloads the connection between the threaded focus ring and the barrel in a very positive way. The screws are loosened when focus adjustments are made, and then tightened to secure the adjustment. Each screw is equipped with a large knob for ease of use.

\subsubsection{Dewars}

Larger CCDs, larger field flatteners, and new readout electronics necessitated a redesign of the dewars for BOSS. The new design borrows considerably from SDSS with the front half of the dewar containing the field flatteners, the detector, and the pre-amp board, and the rear half of the dewar containing the $\mathrm{LN}_{2}$ reservoir. However, unlike the SDSS configuration, the red and blue detector packages are physically quite different, both from each other and from the original CCDs. The blue channel CCD has a thick silicon carbide package while the fully depleted device used in the red channel is mounted to an invar substrate. This led to a redesign of the detector mounting and adjustment scheme, the red and blue designs being unique. 


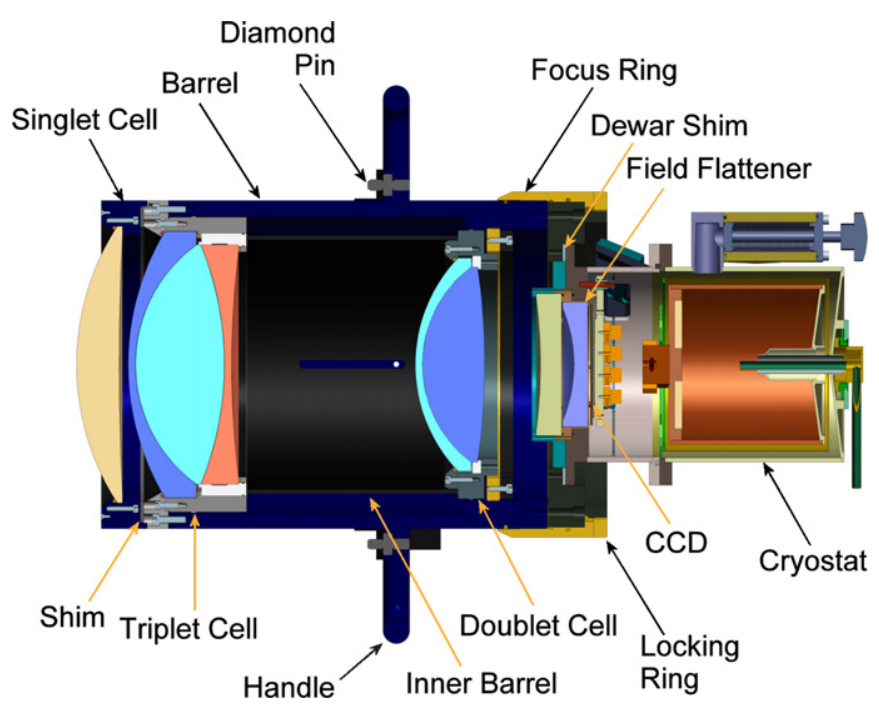

Figure 30. Cross section of the BOSS blue camera. As with the original SDSS design, the red camera opto-mechanical design is identical except for subtle differences in the location of the lens groups.

The design of the $\mathrm{LN}_{2}$ reservoir is nearly identical to the SDSS design. The dewar volume is increased slightly with the usable volume being 0.4 liters. The heat load is approximately $4.5 \mathrm{~W}$, slightly larger than in the original design. With the increased reservoir volume, the hold time is approximately four hours. The dewar is filled automatically at two hour intervals.

\subsubsection{Dewar Autofill System}

BOSS uses the same autofill system implemented for SDSS, with one minor exception. About a year after the start of BOSS, once the imaging camera was decommissioned, the 180 liter tank used to cool the imager was re-purposed for cooling one of the spectrographs. Hence, the current autofill configuration uses two 180 liter supply dewars, one dedicated to each spectrograph.

\subsection{Flexure}

Given the measured flexure for SDSS (see Section 4.3), smaller fibers, and smaller CCD pixels, the flexure expected for BOSS is 0.5 pixels for $15^{\circ}$ on the sky. This exceeds the 0.3 pixel goal set for SDSS by a modest amount. However, the acceptable tolerances (spectral and spatial) for mechanical flexure are, in practical terms, determined by the extent to which they can be compensated for in the data reduction.

Based on the method developed for SDSS (see Section 4.1), we considered 0.5 pixels of flexure to be acceptable. If we consider a maximal $15^{\circ}$ rotation per hour, then we have a fiducial shift of 0.13 pixels in the spectral direction and 0.13 pixels in the spatial direction over the course of a single 15 minute exposure. The magnitude of the blurring introduced by this flexure within an exposure will be given by the total shift scaled by $1 / \sqrt{12}$, which corresponds to the rms of a tophat function. When added in quadrature to the $\sim 1$ pixel dispersion of the spectrograph PSF, this is a fractional effect of $7 \times 10^{-4}$, an insignificant effect on the resolution of the spectra. It also does not degrade the quality of near-infrared sky subtraction, since the sky-modeling algorithm does not rely on any calibrated estimate of the spectral resolution for sky lines, but rather implicitly fits for the spectral resolution as a low-order function of fiber number simultaneously with the fit for the sky spectrum in each exposure. In the spatial direction, this introduces only a negligible mismatch with the

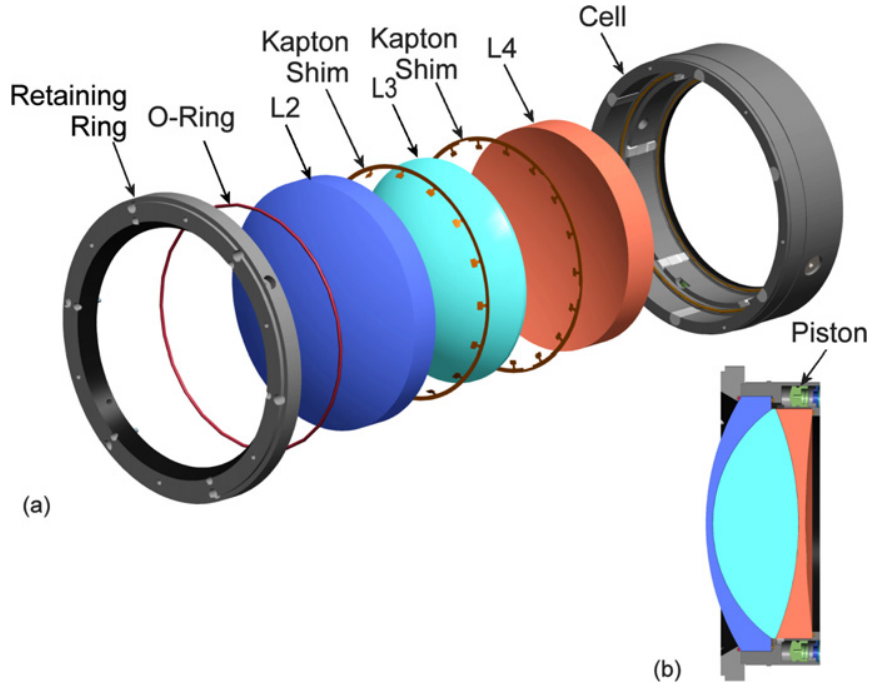

Figure 31. (a) Exploded view of the BOSS camera triplet, and (b) a crosssection of the BOSS triplet. In this revised design, the lenses are coupled by Cargille Laser Liquid 1074. Lenses are spaced using $0.25 \mathrm{~mm}$ Kapton shims with fingers that extend radially inward near the lens outer diameters. Gaps between the fingers allow fluid to flow into the gap between the lenses. O-rings seal the liquid volume at the rear face of the last element and at the rear face of the front element.

cross-sectional profile measured from flat-field exposures that is used to extract the science spectra.

\subsection{Detectors, Electronics, and Data Acquisition Upgrades}

\subsubsection{Detectors}

Modern CCDs with higher QE and smaller pixels were installed for BOSS. On the blue side of the spectrograph, e $2 \mathrm{v}^{59}$ CCDs were installed for improved QE in the far blue while fully depleted devices from Lawrence Berkeley National Laboratory (LBNL) were installed for improved QE near $10000 \AA$ (Holland et al. 2006). With some improvements in the optics as described previously, the $15 \mu \mathrm{m}$ pixel size provides optimal 3 pixel sampling of the PSF and the larger format allows for larger gaps between v-groove blocks to sample the wings of the PSF as well as additional pixels in the dispersion direction to accommodate the larger range in wavelength. Equally important, as evidenced in a comparison of Figure 12 to Figure 28, the upgrade to the new devices provides substantially improved QE in the regions $3560 \AA<\lambda<5000 \AA$ and $7000 \AA<\lambda<10400 \AA$.

\subsubsection{Readout Electronics}

The readout electronics are almost unchanged from the earlier system, with some additions and modifications required for driving the p-channel LBNL CCDs and handling the larger format of both new detectors. A new dewar board incorporating the preamp (now four channels, one for each quadrant of the new detectors) and clock drivers was designed and built, one version each for the $\mathrm{n}$-channel blue CCDs and the $\mathrm{p}$-channel red CCDs. The Burr-Brown OPA627s were replaced with Analog Devices AD8610s, ${ }^{60}$ which are slightly noisier but faster and have lower power dissipation; the gain of the new devices is so much higher than that of the earlier CCDs that the slightly higher noise is of no consequence, and the signal chain adds less than a third of an electron in quadrature to the system noise. New and

\footnotetext{
59 e2v Technologies, http://www.e2v.com

60 Analog Devices, http://www.analog.com
} 
more modern analog switches were incorporated as well, partly to better incorporate the new faster readout ( 88 kilopixels per second), which is twice as fast as the one used previously. Some of the SDSS camera devices had only one good amplifier and had to be read at this rate, so the signal chain was designed to handle it from the beginning, but better and faster switches make it work better. With the $88 \mathrm{kHz}$ rate, the larger format devices with four channels are read in the same time as the earlier SDSS devices with two.

The dewars are equipped with two hermetic micro-D connectors, one with 51 pins for DC and video, and another with 37 pins for digital clock signals. Cables lead from connectors to saddlebags similar to the originals, but with more boards. The original bus receivers, power-distribution, and signal-chain/bias boards are used with a few gain-setting component changes, but now two sig/bias boards are used, since each was built to handle only two outputs. For the "upside down" LBNL p-channel device, a new board that inverts the bias voltages was designed and built. This board also handles the control of the large substrate bias voltage, which places a large DC potential across the device to minimize charge diffusion.

The data and control between the saddlebags and the controller are handled on two cables, a standard 68 pin SCSI cable carrying the RS485 clock signals and a few slow CMOS signals, and a 50 pin MDM cable carrying the digitized video, ADC clocks, and the new voltages.

The controllers are substantially modified. A new power supply to generate the large negative voltages $(-30 \mathrm{~V})$ for VDD and VRD and the very high positive substrate bias $(+100 \mathrm{~V})$ for the LBNL devices was added. The single-micro topology was changed to the camera's two-micro architecture to accommodate the more complex operating environment using two rather different kinds of devices. The clocks are generated synchronously for both red and blue CCDs, but the two sets of clocks themselves are completely different for the two CCD types. The architectures of the detectors are different in detail but also grossly - the e $2 \mathrm{v}$ blue devices are four-phase in the parallels, but the LBNL devices are three-phase, and the way charge is passed to the serial register is rather different.

The FOXIs have been replaced by a fast Ethernet-interfaced micro, the Netburner, described in the next subsection.

\subsubsection{Data Acquisition}

The data acquisition (DAQ) system is responsible for data transfer from the spectrograph to the instrument control computer (ICC), located approximately 300 feet from the telescope. The DAQ receives pixel data from the spectrograph electronics, performs some processing including simple data integrity tests, formats the data according to the FITS standard, and saves the images on a disk array. These operations have to be performed in real time to maximize throughput and overall survey efficiency. The architecture of the BOSS DAQ is similar to the proven design of the original SDSS DAQ, described in Section 2.6. The main change was the use of standard commodity computing components to replace the original, aging hardware modules, many of them custom-built and no longer supported. An additional benefit was the replacement of licensed software packages with free, open-source products.

A block diagram of the BOSS DAQ system is shown in Figure 32. Separate DAQ modules connect to the digitizer boards of the two cameras in each of the spectrographs. Copper Ethernet cables connect the DAQ modules to a network switch mounted on the telescope, where the data is converted to optical signals and travels over optical fibers to the computer housed in a separate building.

$D A Q$ hardware. To efficiently collect, package, monitor, and transmit data from the BOSS spectrographs, a custom printed circuit card was designed. The card is called the DAQ board and a block diagram of its contents is shown in Figure 33. One DAQ board is required for each BOSS spectrograph. During normal operation, the DAQ board receives eight synchronous streams of pixel data, coming from the two cameras on each spectrograph, each camera having one CCD with four outputs. The incoming data, in RS-485 format, are stored in a frame buffer to decouple input and output operations, reformatted and transferred via a 100 Mbit Ethernet link to the ICC over optical fiber to the computer room. The input stage of the DAQ board operates at the pixel clock rate set by the front-end electronics. The buffer memory is of sufficient size to store an entire exposure, but under normal circumstances the network interface is fast enough to keep up with the input rate. In order to fit in the limited space available on the spectrograph, the DAQ card was designed to be compact, $65 \times 115 \mathrm{~mm}$, and to operate from a single $5 \mathrm{~V}$ supply. The design of the DAQ card is based on three main components; a Xilinx FPGA, a NetBurner Ethernet Core Module, and eight $8 \mathrm{M} \times 16$ pseudo-static ram chips (PSRAM). An overview of these components and their functions is given below.

The Xilinx FPGA, a Spartan XC3S1400AN, ${ }^{61}$ controls the input stage of the DAQ module and the frame buffer memory. The bus interface to the NetBurner module is also implemented in the FPGA. The pixel data streams are de-serialized and recorded in the frame buffer which is organized as a First-In/First-Out (FiFo) memory. The FPGA is configured to write to all eight memory modules simultaneously, thus maximizing the data recording speed. At the FPGA level data integrity checks are performed by keeping pixel and line counts. The acquisition software running on the NetBurner module can access this information and, if necessary, raise appropriate flags in the ICC. For testing and debugging purposes the FPGA also contains logic to emulate the spectrograph signals and load test images to the frame buffer, which in turn can be read and sent to the ICC. With this feature, problems upstream from the DAQ card may be located and the ICC and analysis code can be debugged easily using well-defined images. In addition, the FPGA firmware includes a watchdog timer that will raise a flag if the camera data is not loaded into the DAQ card within a reasonable time limit.

The NetBurner Ethernet Core Module, model 5234, ${ }^{62}$ coordinates the communication between the DAQ card and the ICC. The software for the NetBurner is written in $\mathrm{C} / \mathrm{C}^{++}$and will be discussed in detail in the following section. The NetBurner contains a Freescale ColdFire 5234 microprocessor, 2 MB Flash memory, 8 MBytes of SDRAM, and 10/100 Ethernet interface. The ColdFire microprocessor has a full 32 bit architecture providing more than 140 million instructions per second of processing power. The NetBurner module is interfaced to the FPGA via a 16 bit external data bus, which provides a simple and fast communication link.

The $8 \mathrm{M} \times 16$ pseudo-static DRAM ram chips used to buffer and store the spectrograph images are Micron MT45W8MW16BGX. ${ }^{63}$ Pseudo-static PSRAM is a memory technology that features an SRAM-like architecture using a

\footnotetext{
61 Xilinx, Inc., http://www.xilinx.com

62 NetBurner, Inc., http://www.netburner.com

63 Micron Technology, Inc., http://www.micron.com
} 


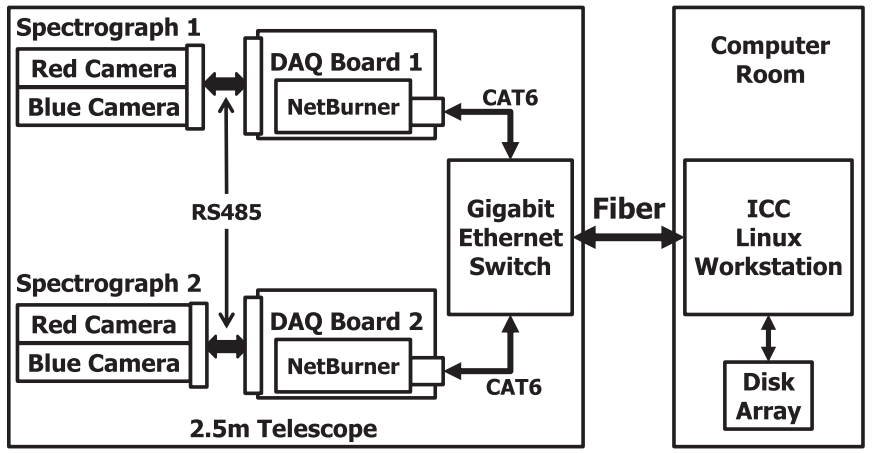

Figure 32. Block diagram of the upgraded BOSS DAQ system.

DRAM cell with internal refresh operations that do not interfere with user read or write cycles. This approach significantly reduces the complexity of the DAQ board while still allowing for sufficient memory to buffer an entire exposure. Should a problem arise with the communication link to the ICC, this feature allows the data to be resent repeatedly until it is received correctly. The PSRAM type chosen is limited to a read/write timing of $70 \mathrm{~ns}$, but consumes little power; less than $50 \mathrm{~mA}$, during a read or write cycle.

$D A Q$ software. The DAQ software consists of two major pieces. The acquisition part, written in $C$, runs on the NetBurner modules on each of the DAQ boards. The second software component, a Python module, provides image formatting as well as an interface to the BOSS online system. This software is executed on the instrument control computer in the computer room. The acquisition program takes advantage of the soft real time functionality provided by the NetBurner software tools. One task implements a command interface and support for the emulation mode used for hardware and software debugging. A second task is responsible for reading out the data from the frame buffer on the DAQ module and for data transmission to the ICC using the TCP/IP protocol. Additional functionality provided by the application includes a separate reset connection to restart the software in case of problems, as well as telnet support to allow remote connections to the NetBurner low-level debugger. Furthermore, the NetBurner allows for software updates over $\mathrm{TCP} / \mathrm{IP}$, permitting remote modification and testing in the event that the acquisition software developer is not on site.

Both BOSS spectrographs operate independently at the DAQ level and are seen by the online system as independent devices. A Python module was developed to allow the online system to interact with the DAQ controllers. Responsibilities for the data transfer, connection management and data integrity checks, such as the correct number of pixels, lie with this module. The Python module also rearranges the pixel streams from the four amplifiers for each of the two CCDs, generating FITS formatted files with contiguous data sections and with the overscan regions moved to the edges of the array. Header keywords are generated and added, and for each camera a separate FITS file is written to disk.

$D A Q$ performance. In almost two years of survey operation the BOSS DAQ system has performed efficiently and reliably. The readout time has been measured to be $55.6 \mathrm{~s}$. Only $1.6 \mathrm{~s}$ of this period is added by the DAQ system, while the bulk of the readout time is used for CCD digitization and is set by the clocking speed, which is optimized to minimize read noise.

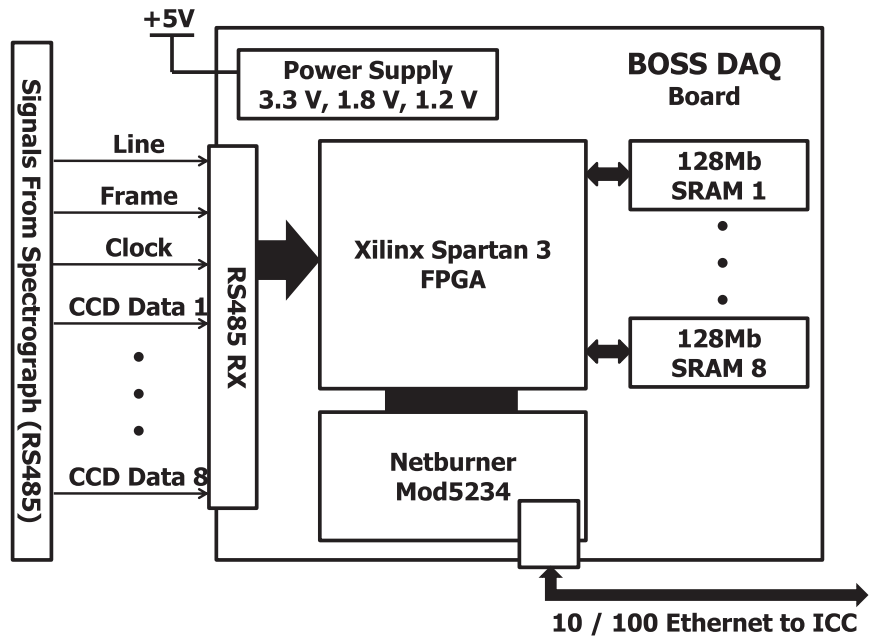

Figure 33. Block diagram of the BOSS DAQ card.

\subsection{BOSS Calibration}

The calibration system described in Section 2.7 was largely preserved for calibration in BOSS with the exception of two minor changes. The neon lamp was replaced by a carefully constructed neon-argon tube ${ }^{64}$ to provide argon lines farther to the red than the neon spectrum reaches. Pixel-to-pixel variation was mapped using a "lossy-fiber" that was placed in the position of the usual fibers along the slithead of an engineering cartridge. This fiber was acquired from MeshTel (model\# GF-400-1-SMA) ${ }^{65}$ and is illuminated by a Deuterium Tungsten Halogen light source provided by Ocean Optics (model\# DTMini-2-GS) ${ }^{66}$ It is designed with a diffusive cladding and transmittive coating so that it glows uniformly along its length. While the usual flat fields are illuminated through discrete fibers, the lossy-fiber spectra is aligned with the slit so that it produces a similar spectral dependence but with nearly uniform illumination in the spatial direction.

\subsection{Guider Upgrade}

\subsubsection{Overview}

As mentioned in Section 2.8, the original Roper Scientific camera used for the guider in SDSS-I and SDSS-II was unsatisfactory on several counts, and it was decided at the outset of SDSS-III to replace it with a cooled camera with a better detector. In addition, although the pointing of the telescope is usually accurate enough that guide stars are found in two large $\left(11^{\prime \prime}\right)$ guide fibers, the efficiency of the survey would be enhanced if we had larger acquisition fibers, and we implemented two of these per cartridge in SDSS-III.

With the original SDSS system, focus was achieved by trial and error, adjusting focus of the telescope and tracking changes in the FWHM of the stars in the guide fibers. The focus system for the SDSS imaging camera worked quite well. In this system, stars were simultaneously imaged onto one focal plane that was set some distance behind the scan data and another set of stars were imaged onto a focal plane that was set some distance in front of the scan data. From the images mapped in these three focal planes, it was possible to derive a focus error signal, and it was decided to implement a similar design in the new

\footnotetext{
${ }^{64}$ Custom made by Absolutely Neon, Inc., http://www.absolutelyneon.com

65 MeshTel INTELITE, Inc., http://www.meshtel.com

66 Ocean Optics, Inc., http://www.oceanoptics.com
} 
spectroscopic guide system for SDSS-III. In a similar fashion to the imaging system, a set of guide fibers was placed in the same focal plane as the science fibers for the spectrograph, a second set was slightly displaced outside of focus, and a third set inside. In addition to the two large acquisition fibers, the number of guide fibers was increased from 11 to 14 to account for any potential decrease in sensitivity in the unfocused fibers to perform the other guider functions.

\subsubsection{Guider Camera}

The new guider camera is an Alta E47 from Apogee Imaging Systems. ${ }^{67}$ The camera employs a $100 \mathrm{Mb} \mathrm{s}^{-1}$ Ethernet interface, uses a thermoelectrically cooled e2v CCD47-10 AIMO $1024 \times 1024$ back-illuminated CCD with $13.5 \mu \mathrm{m}$ pixels, normally binned $2 \times 2$. This results in $27 \mu \mathrm{m}, 0$.'45 pixels. The images are nearly always well-sampled for the seeing conditions at APO. The camera is much more sensitive and stable than the old one, and allows the use of much fainter guide stars.

The camera was supplied with a liquid cooling head, which we replaced with a similar part made of copper. The replacement is intended to alleviate prior bad experiences in the glycol system with aluminum parts. Otherwise, the camera is used as supplied. The reimaging system from the output fiber block to the CCD and the broad visual (BG38) filter from the old system was retained for the new guider.

\subsubsection{Guide Fibers}

The new system uses 14, $8^{\prime \prime}(500 \mu \mathrm{m})$ Sumitomo (model\# IGN-05/10) coherent fibers of the same kind used in the old system for guiding. Eight of these are in ferrules which place them at the nominal focus of the plate, and three each are $200 \mu \mathrm{m}$ inside and outside of nominal focus. The focus offset adds 0 '.7 FWHM in quadrature to the seeing of the images, which is not yet enough to make them unsatisfactory for guiding, but still provides a sensitive measure of focus.

In addition to the 14 guide fibers, there are two much larger $24^{\prime \prime}(1.5 \mathrm{~mm})$ acquisition fibers, which are inexpensive coherent plastic fiber bundles manufactured by NanOptics (model\# B29060J-175), ${ }^{68}$ used for guide star acquisition only. Though not used for guiding, their quality seems to be almost as good as the glass guide fibers.

\subsubsection{Software, Control, and Performance}

The software for the new control system is an adaptation of the old guider code, written mostly in $\mathrm{C}$. There are a few enhancements over the old system, including a fast acquisition mode that uses the acquisition fibers only, a mode in which successive guider images can be accumulated to improve stability when using bright guide stars and therefore short exposures, and a slow servo loop to actively and automatically control the scale of the telescope (in the SDSS-I/II surveys the scale of the telescope was adjusted manually). The upgraded BOSS guider automatically tracks in R.A., decl., rotation, and scale. All of the fits used to generate guider offsets are simple least-square deviation fits; it is possible that we could do better if a refraction model using the actual distribution of the guide stars on the plate were used, but this has never been implemented. The present system is satisfactory if the guide stars are reasonably uniformly distributed over the plate; we strive to achieve this distribution in the process of designing each plate.

\footnotetext{
67 Apogee Imaging Systems, http://www.ccd.com

68 NanOptics, Inc., http://www.nanoptics.com
}

The display code was completely rewritten for the new system but is functionally identical to the old one. A "raw" CCD image is displayed to the observers as is an image in which the fiber images are distributed approximately as they are on the sky. This display makes qualitative evaluation of the guider behavior easy. In addition, the guider errors are displayed for each fiber for each image and are recorded in the guider frame headers.

\section{SDSS AND BOSS SPECTROGRAPH PERFORMANCE}

Here we describe the methods for determining the instrument performance for SDSS and BOSS in relation to the predictions outlined earlier in the paper. A summary of the design predictions and measured performance is presented in Table 3. Instrument characterization is presented in three parts: the spectroscopic pipeline, the spectrograph performance, and CCD performance. Data found in DR8 (Aihara et al. 2011) are used in the characterization of the SDSS spectrograph and data in DR9 (Ahn et al. 2012) are used in the characterization of BOSS.

\subsection{Spectroscopic Pipeline}

The spectroscopic redshifts and classification for SDSS and BOSS are based on the data reduction pipeline idlspec $2 \mathrm{~d} .{ }^{69} \mathrm{~A}$ brief explanation of the routines for SDSS is found in AdelmanMcCarthy et al. (2008) and Aihara et al. (2011). SDSS data were processed using idlspec2d v5_3_12, which is the same version of the software used in DR8. The pipeline was modified for BOSS to account for the increase in wavelength coverage, number of fibers, and other changes discussed in Section 3. For a brief description of the BOSS software reductions, see Dawson et al. (2013) and for a full description of the automated classification, see Bolton et al. (2012). BOSS data were processed using v5_4_31, a somewhat earlier version of the v5_4_45 software used for DR9 (Ahn et al. 2012). There are minor changes between these two versions, but the differences do not change the conclusions of the work presented here. The intermediate data products produced by the idlspec $2 \mathrm{~d}$ pipeline are used to characterize the instrument performance; we include a brief explanation of the relevant functions here.

SDSS and BOSS observations of each plate follow the sequence: mount cartridge, slew to field, focus spectrograph, take exposure with calibration arc lamps and flat field lamps, and obtain a series of 15 minute science exposures. In the first step of the data reduction, the read noise and bias level from the raw images is determined from the overscan of the image. Next, raw images are bias-subtracted and counts are converted into electrons using CCD amplifier gains specific to each quadrant of each detector. Cosmic rays are then identified using a filter to discriminate between the sharp edges of cosmic ray trails and the smoother profile of optical sources. The noise of each pixel is determined by taking the Poisson noise from the electron counts in quadrature with the measured read noise. Finally, a flat-field image is used to correct the pixel-to-pixel response in the CCD.

Each fiber projects a spectrum onto the CCD with an rms in the spatial direction of roughly 1 pixel and a spectral dispersion of roughly 1 pixel rms in the direction of the parallel clocking. The data from the flat field exposures are used to model the profile of each fiber on the CCD and also to normalize fiber-tofiber throughput variations. The data from the arc exposures are used to determine the wavelength solution for each fiber.

\footnotetext{
69 Current and development versions of the software are found at http://www.sdss3.org/dr9/software/products.php.
} 
Table 3

Design Predictions and Measured Performance for SDSS and BOSS

\begin{tabular}{|c|c|c|c|c|}
\hline \multirow[b]{2}{*}{ Quantity } & \multicolumn{2}{|c|}{ SDSS } & \multicolumn{2}{|c|}{ BOSS } \\
\hline & Design & Measured & Design & Measured \\
\hline \multicolumn{5}{|c|}{ Spectrograph 1} \\
\hline Wavelength range & $3900-9100 \AA$ & $3800-9220 \AA$ & $3560-10400 \AA$ & $3560-10400 \AA$ \\
\hline Resolving power $(\lambda<3800 \AA)$ & $\mathrm{N} / \mathrm{A}$ & $\mathrm{N} / \mathrm{A}$ & 1000 & $1220-1480$ \\
\hline Resolving power $(3800 \AA<\lambda<4900 \AA)$ & 1500 & $1500-1900$ & 1400 & $1200-1900$ \\
\hline Resolving power $(\lambda>4900 \AA)$ & 1500 & $1900-2600$ & 1000 & $1550-2550$ \\
\hline Total throughput (4000 ̊̊) & 0.11 & 0.08 & 0.18 & 0.15 \\
\hline Total throughput (6000 ̊̊) & 0.20 & 0.17 & 0.25 & 0.21 \\
\hline Total throughput (8000 ̊̊) & 0.14 & 0.13 & 0.29 & 0.25 \\
\hline Total throughput (10000 ̊̊) & $\mathrm{N} / \mathrm{A}$ & $\mathrm{N} / \mathrm{A}$ & 0.12 & 0.10 \\
\hline Flexure: blue channel, spectral (pixels per $15^{\circ}$ on the sky) & 0.3 & 0.33 & 0.5 & 0.40 \\
\hline Flexure: blue channel, spatial (pixels per $15^{\circ}$ on the sky) & 0.3 & 0.18 & 0.5 & 0.11 \\
\hline Flexure: red channel, spectral (pixels per $15^{\circ}$ on the sky) & 0.3 & 0.30 & 0.5 & 0.41 \\
\hline Flexure: red channel, spatial (pixels per $15^{\circ}$ on the sky) & 0.3 & 0.21 & 0.5 & 0.18 \\
\hline \multicolumn{5}{|c|}{ Spectrograph 2} \\
\hline Wavelength range & $3900-9100 \AA$ & $3800-9220 \AA$ & $3560-10400 \AA$ & $3560-10400 \AA$ \\
\hline Resolving power $(\lambda<3800 \AA)$ & $\mathrm{N} / \mathrm{A}$ & $\mathrm{N} / \mathrm{A}$ & 1000 & 1300 \\
\hline Resolving power $(3800 \AA<\lambda<4900 \AA)$ & 1000 & $1700-2200$ & 1400 & $1350-1950$ \\
\hline Resolving power $(\lambda>4900 \AA)$ & 1000 & $1850-2650$ & 1000 & $1700-2600$ \\
\hline Total throughput (4000 ̊̊) & 0.11 & 0.09 & 0.18 & 0.14 \\
\hline Total throughput (6000 ̊) & 0.20 & 0.18 & 0.25 & 0.24 \\
\hline Total throughput (8000 ̊̊) & 0.14 & 0.12 & 0.29 & 0.25 \\
\hline Total throughput (10000 ̊) & $\mathrm{N} / \mathrm{A}$ & $\mathrm{N} / \mathrm{A}$ & 0.12 & 0.10 \\
\hline Flexure: blue channel, spectral (pixels per $15^{\circ}$ on the sky) & 0.3 & 0.30 & 0.5 & 0.43 \\
\hline Flexure: blue channel, spatial (pixels per $15^{\circ}$ on the sky) & 0.3 & 0.31 & 0.5 & 0.07 \\
\hline Flexure: red channel, spectral (pixels per $15^{\circ}$ on the sky) & 0.3 & 0.27 & 0.5 & 0.44 \\
\hline Flexure: red channel, spatial (pixels per $15^{\circ}$ on the sky) & 0.3 & 0.25 & 0.5 & 0.15 \\
\hline
\end{tabular}

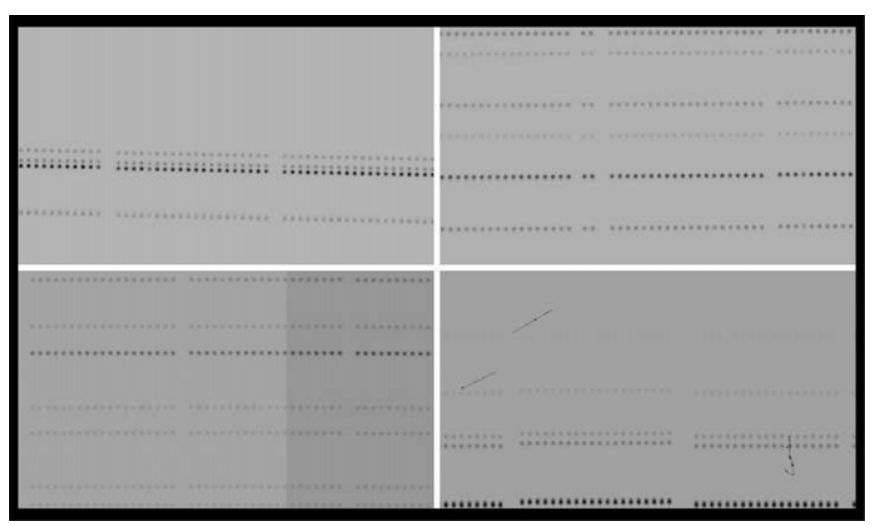

Figure 34. Raw arc image taken with the BOSS spectrograph. Top left panel: 3650-3850 Å on blue CCD; top right panel: 5050-5250 A on blue CCD; bottom left panel: 6000-6250 ̊̊ on red CCD; bottom right panel: 8850-9100 ̊ on red CCD. Each panel covers roughly 50 spectra.

Figure 34 shows an example of the arc lines obtained in a BOSS exposure. The top left panel is taken near the edge of the blue CCD and shows a slight tilt in the wavelength direction due to optical distortion. This image also demonstrates the bundling of 20 fibers separated by a gap on either side. The top right panel demonstrates a dropped fiber near the right edge of the first bundle. The bottom left image from a red camera is taken near the center of the CCD and shows the amplifier gain between two quadrants at a different background count. The lower right panel shows several cosmic rays interacting with the CCD on a red camera. The bottom right image also shows an offset in the wavelength direction between the central bundle and the surrounding bundles due to imperfections in the slithead assembly. The top two images are more representative of the SDSS CCDs because the red BOSS CCDs are much thicker and lead to more pronounced cosmic ray trails. We use the wavelength solution and line-spread function derived from the arc exposures to determine wavelength coverage and resolution.

Each two-dimensional science image is collapsed into a series of one-dimensional spectra using the fitted profile and wavelength solution from the calibration exposures for each fiber. At this point, the "SKY" fibers (fibers assigned to areas with no detected objects) are used to model the background as a function of position on the detector. The model sky background is subtracted from the one-dimensional spectra. These one-dimensional spectra contain all of the information required to characterize the instrument performance. For science applications, the spectrum from each fiber is resampled to a pixel size of $69 \mathrm{~km} \mathrm{~s}^{-1}$ and combined with the spectra from the same fiber for each exposure in the sequence to generate a high signal-to-noise spectrum. Redshifts and object classification are determined from these co-added spectra as is described in Bolton et al. (2012).

The data reduction pipeline also compensates for spectral shifts due to mechanical flexure. Shifts between exposures in the spatial direction are compensated by iteratively re-centering the traces of all fibers starting from the positions determined from flat-field exposures, and adopting the median spatial shift as a global value for each exposure. For this process to converge properly, the accumulated spatial shift between flat and science frames must be significantly less than one-half the typical fiberto-fiber spatial separation, a condition that is satisfied for any reasonable number of science exposures in sequence. Shifts between exposures in the spectral direction are compensated 
by measuring the positions of known bright sky emission lines and fitting a shift-plus-scale for each fiber and each exposure relative to the wavelength solution determined from arc-lamp calibration frames. The ultimate validation of the combined hardware-software system for flexure control is the $\sim 1 \%$ level of systematic residual errors in near-infrared sky subtraction achieved for BOSS (Bolton et al. 2012).

\subsection{Spectrograph Performance}

\subsubsection{Wavelength Coverage}

The SDSS spectrographs have excellent wavelength calibration between the $\mathrm{Hg}$ I arc line at $3901.87 \AA$ and the $\mathrm{Ne}$ I arc line at $9148.67 \AA$. The BOSS spectrographs have excellent wavelength calibration between the Cd I arc line at $3610.51 \AA$ and the $\mathrm{Hg}$ arc line at $10140 \AA$. In between these detected arc lines for SDSS (3902-9149 $\AA$ ) and BOSS (3611-10140 $\AA$ ), the wavelength solutions are typically accurate to $3 \mathrm{~km} \mathrm{~s}^{-1}$. Beyond those wavelengths the solutions are extrapolated and the accuracy has not been evaluated.

The scientific rationale behind the wavelength coverage for the SDSS and BOSS surveys was described in Sections 2.1 and 3.1, respectively. Including the extrapolation beyond the prominent arc lines, the wavelength range included in data reductions for the SDSS spectrographs is 3800-9220 $\AA$. This is slightly broader than the design wavelength range of 3900-9100 $\AA$. The BOSS spectrographs have useful data in the 3560-10400 A wavelength range that are included in all data releases. Although the detectors see light beyond this wavelength range, it is discarded in the reductions since the throughput is very low and the longer wavelengths suffer from second-order light.

Both the SDSS and BOSS blue limits ensure the Ca II $\mathrm{H}$ and $\mathrm{K}$ lines are detected at zero redshift. The BOSS blue limit provides extended coverage of the Ly $\alpha$ forest for quasars at $z>2.2$. The BOSS red limit measures the $\mathrm{Mg} \mathrm{b} 5175 \AA$ and the Na D $5893 \AA$ absorption features for galaxies redshifted to $z<0.72$. The wavelength coverage for SDSS and BOSS leads to detection of at least 31 common astrophysical emission lines, as detailed in Table 5 of Bolton et al. (2012).

\subsubsection{Spectral Resolution}

The spectral resolution is measured from calibration arc images taken before each set of science exposures. The onedimensional arc image is first masked to include only pixels within 12 pixels of the center of each arc line on each fiber. A Gaussian of width $\sigma_{\lambda}$ is fit to each spectral profile using the 25 unmasked pixels. A fourth-order Legendre polynomial model is fit to the derived $\sigma_{\lambda}$ as a function of wavelength to model the dispersion over the full wavelength range. The resolving power for SDSS and BOSS is then formally defined as $R=\frac{\lambda}{2.35 \times \sigma_{\lambda}}$. The resolution is the FWHM of the Gaussian, $2.35 \times \sigma_{\lambda}$.

We measured the resolving power as a function of wavelength from a sample of 100 SDSS plates and 100 BOSS plates. For each plate and each camera, we computed the mean $R$ as a function of wavelength. Figure 35 shows a comparison of the SDSS resolving power to that for BOSS.

The extended coverage at long wavelengths from the new BOSS CCDs is evident redward of $9200 \AA$ while the extended coverage at short wavelengths is more subtle, but also shown in the figure. The presence of a dichroic for SDSS and BOSS is also revealed near $6000 \AA$, where the resolving power no longer increases monotonically with wavelength. The apparent
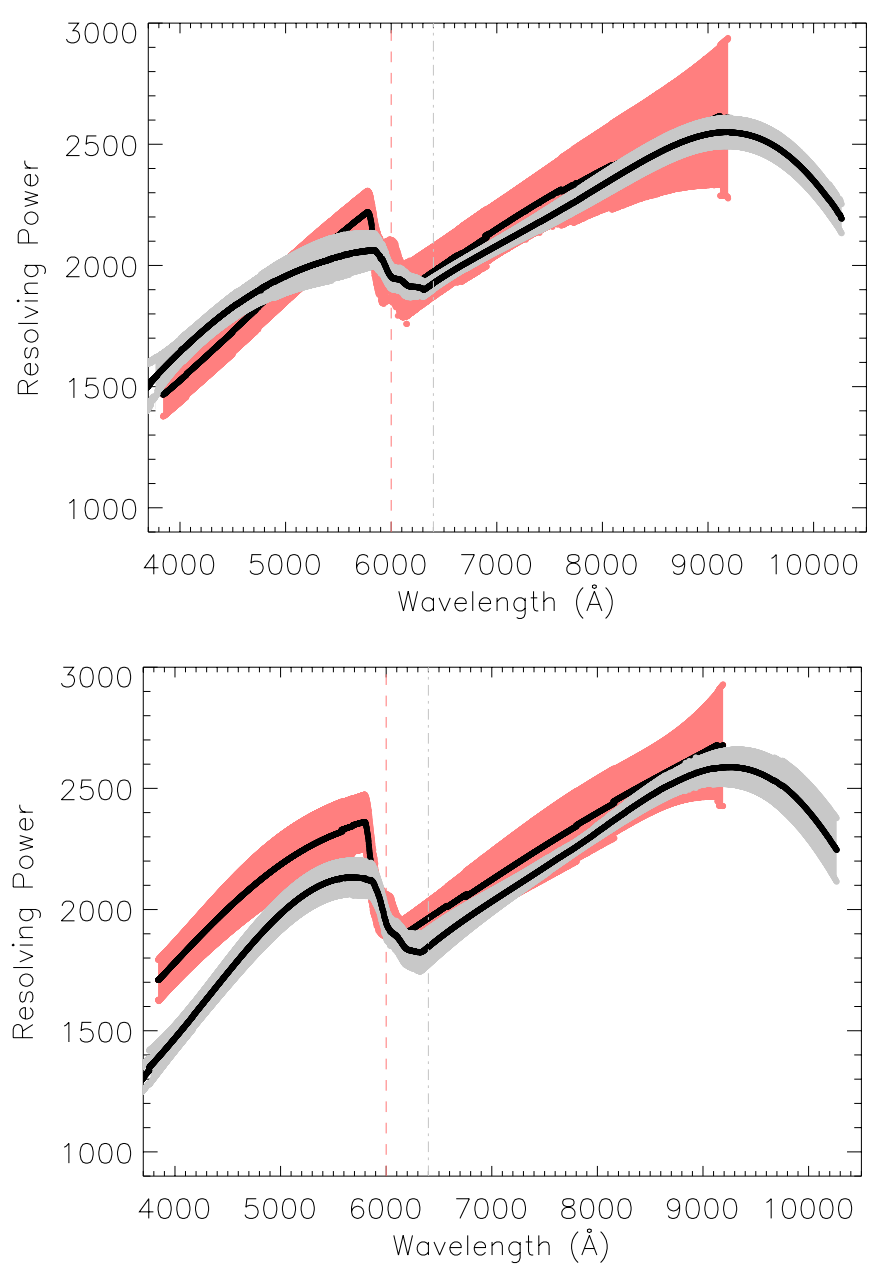

Figure 35. The resolving power for BOSS (gray) and SDSS (red). The shaded regions correspond to the regions that contain $68 \%$ of the plates in the measurement. The top panel shows the results for Spectrograph 1 and the bottom panel shows the results for Spectrograph 2 .

transition around the dichroic is due to the fact that both the red and blue channels contribute to this region, each with different dispersive elements. In the regions where we count flux from both cameras, we simply determine the resolving power by a mean weighted by the relative throughput of the two cameras.

The BOSS requirements and measured resolving power are presented in Figure 36. For BOSS, the position of the fiber on the CCD impacts the resolution due to optical distortion over the larger detectors. The resolving power of the middle fiber is representative of $\sim 80 \%$ of the BOSS fibers, $100 \%$ of the SDSS fibers, and clearly exceeds specifications at all wavelengths for the first spectrograph. However, the fibers near the edge of the first spectrograph have a resolving power that is degraded by up to $20 \%-25 \%$ relative to the central fibers. Also shown in the figure, the two blue channels show quite different patterns of image quality, with most fibers in the second spectrograph missing the requirement for resolving power around $380 \mathrm{~nm}$. The variations in resolution around 4000-5000 $\AA$ are likely due to slight differences in the alignment of the detectors or optics, effects that tend to be amplified at blue wavelengths. A fraction $(\lesssim 25 \%)$ of the BOSS fibers therefore do not meet specifications for spectral resolving power in the wavelength range $3800 \AA<\lambda<4900 \AA$.

In addition to the resolving power as a function of wavelength, we have also determined the rms width $\sigma_{p}$ of the line-spread 

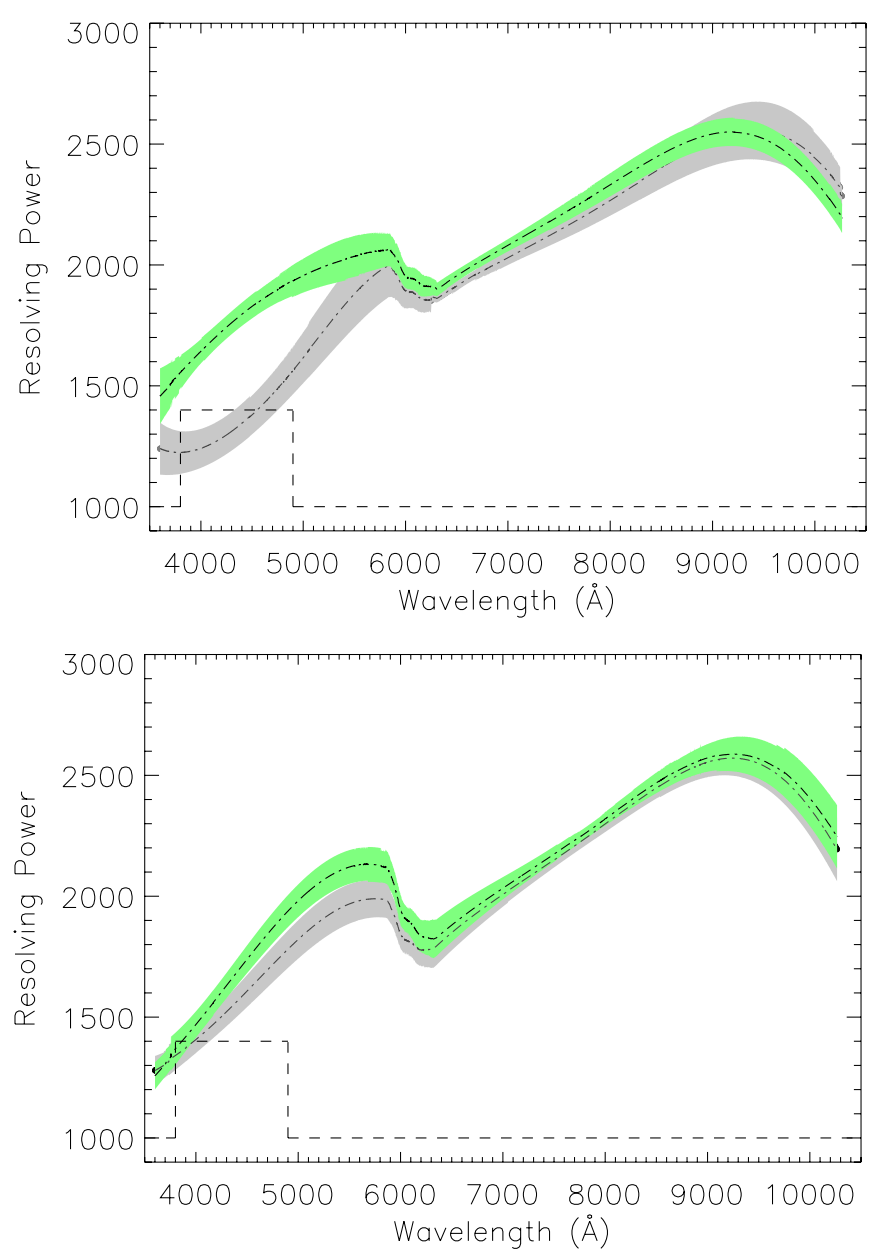

Figure 36. The requirements and the measured resolving power for the two BOSS spectrographs. The results for Spectrograph 1 are shown in the top panel. Results for Spectrograph 2 are shown in the bottom panel. The dashed black curve is the requirement for the resolving power. The green curve is the $68 \%$ confidence limit about the mean of the resolving power for the central fiber and is representative of $\sim 80 \%$ of the fibers. The gray curve is the $68 \%$ confidence limit about the mean of the resolving power for a representative fiber near the edge of the spectrograph slit.

function in native pixels to demonstrate the spatially varying resolution over the focal plane. Figure 37 shows the measured rms pixel resolution of the illuminated region for each of the four BOSS CCDs. For the blue cameras, the spectra near the edges of the detector have a higher $\sigma_{p}$, reflecting the lower optical quality and deviations from flatness of the focal plane at large field angles. The optics project a saddle-shaped focal plane, which contributes to this poorer resolution, an effect that is broadly consistent with predictions in Section 3.3.. The two red cameras display increased rms width between $9500 \AA<\lambda<$ $10200 \AA$, mostly as a consequence of the increased path length of longer wavelength light in the silicon.

\subsubsection{Throughput}

As discussed in Section 3, the primary motivation for rebuilding the spectrographs for BOSS was to increase the instrument throughput. We define the throughput as the ratio of the measured flux for a point source relative to the incoming flux outside the atmosphere. A higher throughput is important for the fainter objects targeted by BOSS, particularly the highest redshift galaxies and the quasars near the magnitude limit of the survey. The throughput described here is the throughput of
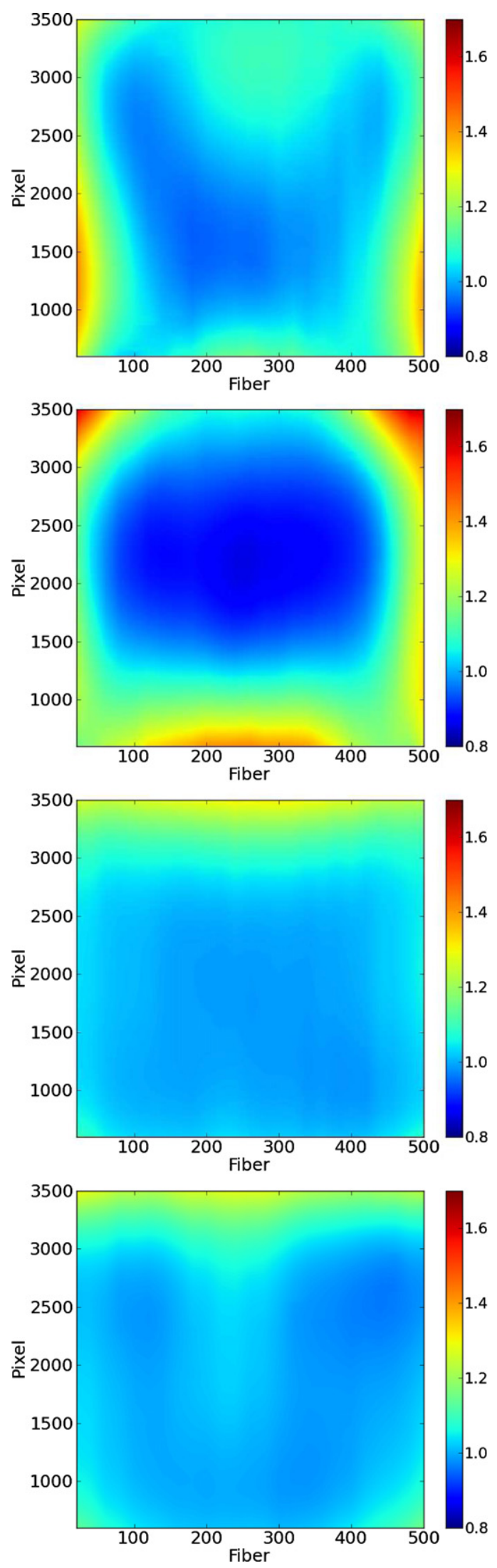

Figure 37. Contour images showing the rms width in pixels in the wavelength direction for each of the four BOSS CCDs. The top panels represent the two blue cameras while the bottom panels represent the two red cameras. In all cases, the wavelength increases from the bottom of the image to the top of the image. The SDSS images have similar characteristics and are not shown here. 

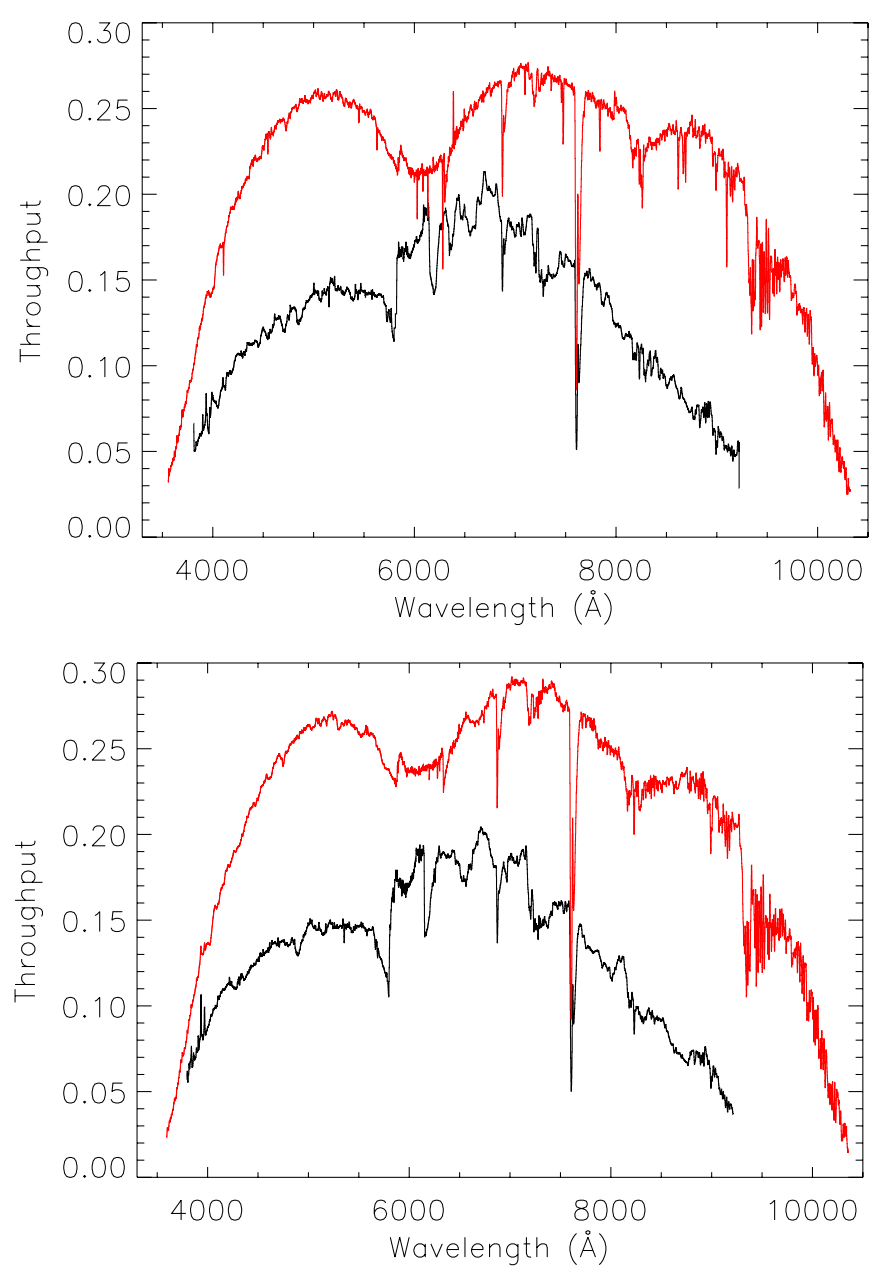

Figure 38. Throughput curves for SDSS (black) and BOSS (red). The results for Spectrograph 1 are displayed in the top panel. The results for Spectrograph 2 in the bottom panel. Note the telluric absorption features.

the entire instrument; the predicted throughput of the individual components was discussed in Sections 2.3 and 3.3.

The throughput is measured from the spectrophotometric standard stars on the plate during science exposures. For each standard star, raw photon counts are measured using a 3 pixel radius boxcar extraction on the calibrated, two-dimensional science frame before flux calibration. The aperture is centered on the spectral trace using the fiber flat-fields from the calibration sequence. The photon counts from the source are estimated at each pixel using the observed magnitudes from the SDSS imaging survey and the synthetic spectral template derived from spectrum. The synthetic spectrum is converted to photons per pixel using the wavelength solution, the effective collecting area of the obstructed primary mirror, and the time of each exposure. The throughput at each pixel is calculated by taking the ratio of the measured raw counts to the number of photons per pixel as predicted by the model.

The throughput for SDSS was measured by averaging the throughput from 84 different standard stars observed under photometric conditions at an airmass of $\sim 1.0$ and seeing $\leqslant 1$ ". 15 . Because BOSS has smaller fibers, the measured throughput on the BOSS spectrographs is more susceptible to guiding errors. We attempt to mitigate this effect by averaging the throughput over the four stars that produce the highest throughput on each camera. The throughput for BOSS was measured by averaging the throughput of 24 stars over three plates, split evenly between

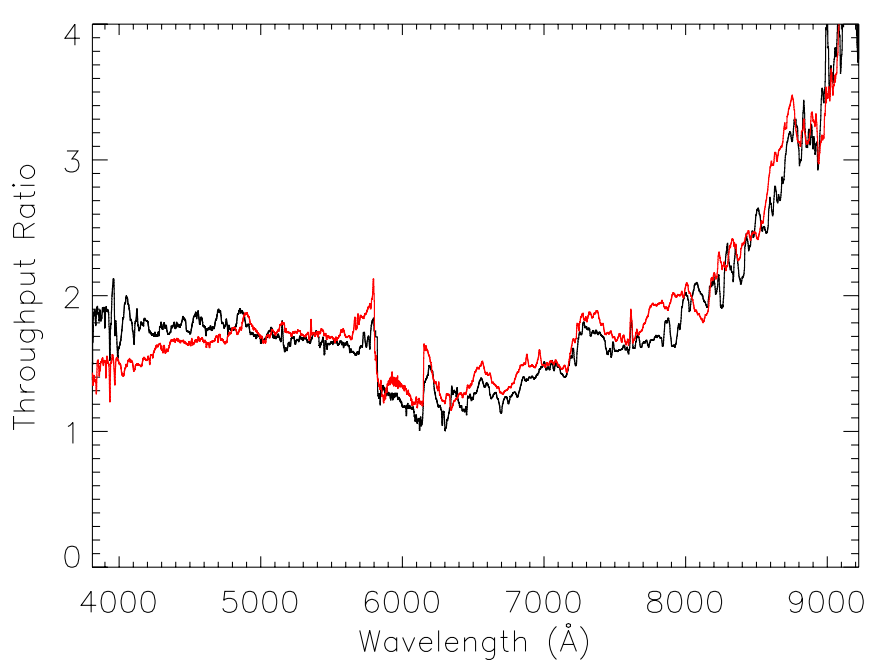

Figure 39. Ratio of throughput BOSS/SDSS after applying a median smoothing kernel of width 100 pixels. The figure displays results for Spectrograph 1 (black) and Spectrograph 2 (red).

the two spectrographs. As with SDSS, we selected standard stars observed under photometric conditions at an airmass of $\sim 1.0$ and seeing $\leqslant 1^{\prime \prime}$. 15 . In both SDSS and BOSS measurements, we correct small differences in seeing by normalizing the observed flux to a double Gaussian PSF (as described in Section 2.3.8) with FWHM of $1^{\prime \prime}$ integrated over an aperture that corresponds to the area of the $2^{\prime \prime}$ or $3^{\prime \prime}$ fiber. The throughput curves have been flat-field corrected to account for fiber-to-fiber variations, thereby normalizing each fiber to the median for each plate.

As shown in Figure 38, the throughput after the BOSS upgrades has been significantly improved. The ratio of BOSS to SDSS throughput is also shown in Figure 39. Not demonstrated here is a fiber dependent variation in the throughput. As in the resolution measurements, fibers near the edge of the BOSS detectors have a slightly lower throughput than fibers near the middle of each spectrograph. For BOSS, there is an additional $\sim 10 \%$ rms variation on the average throughput curve (particularly at short wavelengths) between standard stars. This variation in BOSS is likely caused by scale changes in the telescope over the course of an observation and possible guiding errors (BOSS is more sensitive to guiding because of the smaller fibers). The effect is probably present in the SDSS data as well, but is amplified in BOSS due to the decrease in fiber diameter.

Overall, the SDSS spectrographs delivered on-sky throughput that was consistent between the two instruments to within approximately $10 \%$ across all wavelengths, fell short of design predictions by roughly $10 \%-25 \%$ depending on wavelength, and met the requirements set forth in Section 2.1.6 except at the bluest wavelengths where the $8 \%$ and $9 \%$ delivered throughputs at $4000 \AA$, for Spectrographs 1 and 2, respectively, is compared to the requirement of $10 \%$. The throughput predictions were based on a combination of measured, published, and modeled efficiency curves for a large number of system components, and the agreement with measured throughput to $10 \%-15 \%$ over the majority of the bandpass is satisfying, as is the agreement in overall spectral shape. The largest uncertainty in the throughput prediction is the telescope, which is not well characterized and continually changes with exposure to the environment and periodic cleaning of optical surfaces. The telescope contains two skyward looking surfaces, the primary mirror and the first surface of the dual element wide field corrector. These surfaces, in addition to the secondary mirror, are periodically cleaned with 


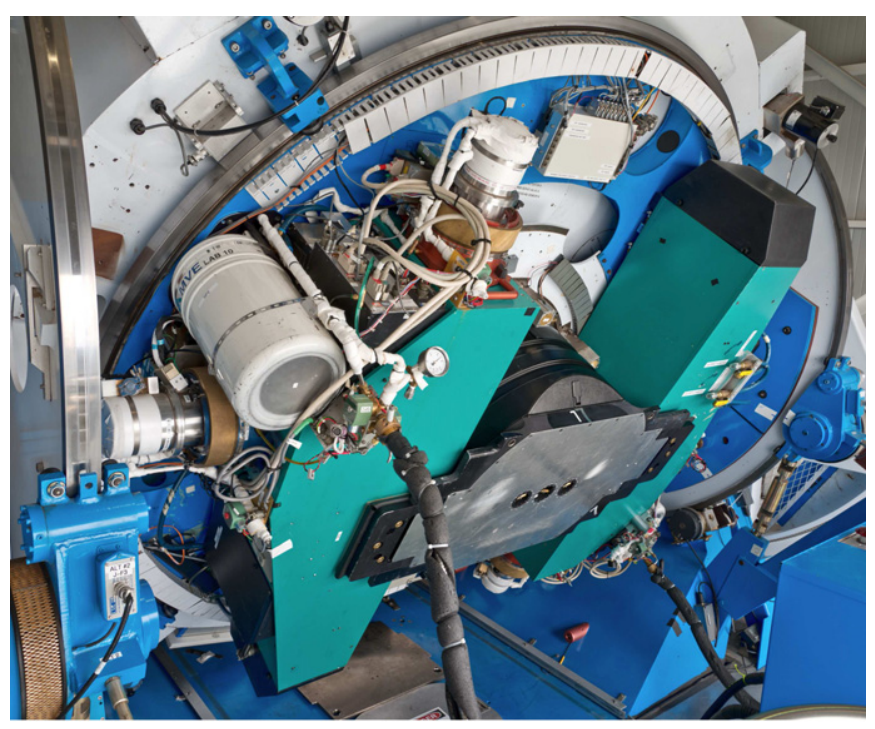

Figure 40. Photograph of the spectrographs mounted to the Cassegrain rotator. Spectrograph 1 is on the right. Spectrograph 2 is on the left. In this orientation the rotator is at zero degrees. Positive rotation is defined as counterclockwise motion as viewed from behind the rotator. Here, the telescope is a zenith angle of $60^{\circ}$

$\mathrm{CO} 2$ snow, which deals with much of the dust but not stickier contaminants such as pollen. This contamination undoubtedly degrades the UV throughput of the telescope more than the longer wavelengths but again, is not well characterized. In addition, the efficiency of several other system components in the UV is rolling off precipitously; thus, the wider discrepancy between predicted and measured throughput in the UV is not unexpected.

The BOSS spectrographs exhibit somewhat more variation in throughput between the two instruments, but not to any degree of surprise or concern; the greatest variation is about $15 \%$ in the dichroic crossover region near $6000 \AA$, while the difference in peak throughput is only $5 \%$. The discrepancy between predicted and measured throughput is flatter across the bandpass than was the case for SDSS, with an average shortfall in measured throughput of roughly $15 \%$. The discrepancy is highest at both ends of the bandpass, but not dramatically so and this may exonerate the telescope throughput model somewhat as a major suspect in the UV discrepancy for SDSS. Again, many component efficiencies are rolling off in the UV and at the other end for BOSS, at $10000 \AA$ the CCD efficiency is falling steeply.

The requirement on BOSS throughput from Section 3.1.6 is a factor of two increase in peak throughput relative to SDSS. While the actual peak throughput was increased by only about $40 \%$, from $21 \%$ to $29 \%$, the throughput was nearly doubled over much of the blue bandpass, and was more than doubled longward of $8000 \AA$.

\subsection{Flexure}

Flexure was characterized for both SDSS spectrographs in 1999. Measurements were taken with the telescope near the horizon at a zenith angle of $72^{\circ}$. A sparsely populated plate was illuminated with $\mathrm{Ne}$ and $\mathrm{HgCd}$ arc lamps to provide an array of spots at mostly random positions on the CCDs. Data were taken approximately every $45^{\circ}$ over the entire $360^{\circ}$ range of the rotator. The sign convention is such that positive rotator angles represent a counterclockwise rotation of the rotator as viewed from behind the rotator. Positive values in the spectral direction represent motion of the spots toward longer wavelengths, and positive values in the spatial direction represent motion of the spectra toward higher column number on the CCD. For reference, a photograph of the spectrographs on the back of the rotator is shown in Figure 40. As shown, the rotator is at the zero degree orientation, and the telescope is at a zenith angle of $60^{\circ}$.

Figure 41 shows image motion as a function of rotator angle for both the spectral and spatial directions in Spectrograph 1. Results for Spectrograph 2 are comparable and a summary can be found in Table 3. At $24 \mu \mathrm{m}$ per pixel, flexure in the spectral direction was measured to be $\sim 0.3$ pixels in the worst case in the red channel and $\sim 0.33$ pixels in the worst case in the blue channel over a $15^{\circ}$ rotation. Total flexure in the spectral direction, over the $360^{\circ}$ rotator range, was measured to be $\sim 3.6$ pixels peak-to-valley in the red channel and $\sim 3.3$ pixels peak-to-valley in the blue channel. In the spatial direction the flexure was lower, about 0.2 pixels in both channels over $15^{\circ}$. Total flexure in the spatial direction was measured to be $\sim 1.4$ pixels and $\sim 1.1$ pixels peak-to-valley in the red and blue channels, respectively. It should be noted that these results are near worst case since most fields observed are much further from the horizon, and flexure is at a maximum at the horizon. Flexure scales as the sine of the zenith angle for instruments on a Cassegrain rotator.

These results are in-line with the performance goal of 0.3 pixels for $15^{\circ}$ on the sky that was discussed in Section 2.5. A noteworthy observation from the results is that the flexure is much worse in the spectral direction, a surprise that should have been anticipated given the collimator mount design. The tall rectangular collimator is supported by a thin membrane fastened to the rear face of the collimator. Having the support behind the center of gravity, combined with the fact that the tip/tilt/piston actuators are not infinitely rigid, allows the mirror to tilt slightly as the gravity vector changes. Considering the contact points of the actuators, the collimator tilts considerably more in the narrow (spectral) direction than in the tall (spatial) direction.

Not long into SDSS operations a subtle problem appeared in the blue channel of one of the spectrographs. It was discovered that the blue camera on Spectrograph 1 (b1), would go out of focus more often than the other cameras. This sudden defocus would tend to happen over a particular range of rotator angle $\left(90^{\circ}-135^{\circ}\right)$, sometimes requiring a refocus of the collimator. Measurements in 2004 indicated the CCD tilted approximately $35 \mu \mathrm{m}$ (corner-to-corner) over a small range of rotator motion but the location of the spectra did not change. In the early phases of BOSS, the problem suddenly got worse, probably due to the increased mass of the BOSS dewars. It was discovered that the focus locking mechanism, by design, did not lock-out the backlash in the focus ring threads. The locking mechanism was redesigned as discussed in Section 3.4.4 and the problem was finally solved.

With the implementation of upgraded cameras and a redesigned focus locking mechanism, flexure was re-characterized for BOSS. Figure 42 shows image motion as a function of rotator angle for both channels of Spectrograph 1. Measurements were taken with the telescope zenith angle at $60^{\circ}$, then scaled to a zenith angle of $72^{\circ}$ for comparison with the results for the SDSS spectrographs. From Figures 41 and 42 it is clear that the new focus locking mechanism reduced flexure overall. Total flexure in the spectral direction, in microns, was reduced from $\sim 85 \mu \mathrm{m}$ peak-to-valley in the red channel of SDSS Spectrograph 1 to $\sim 52 \mu \mathrm{m}$ peak-to-valley in BOSS. Similarly, flexure 

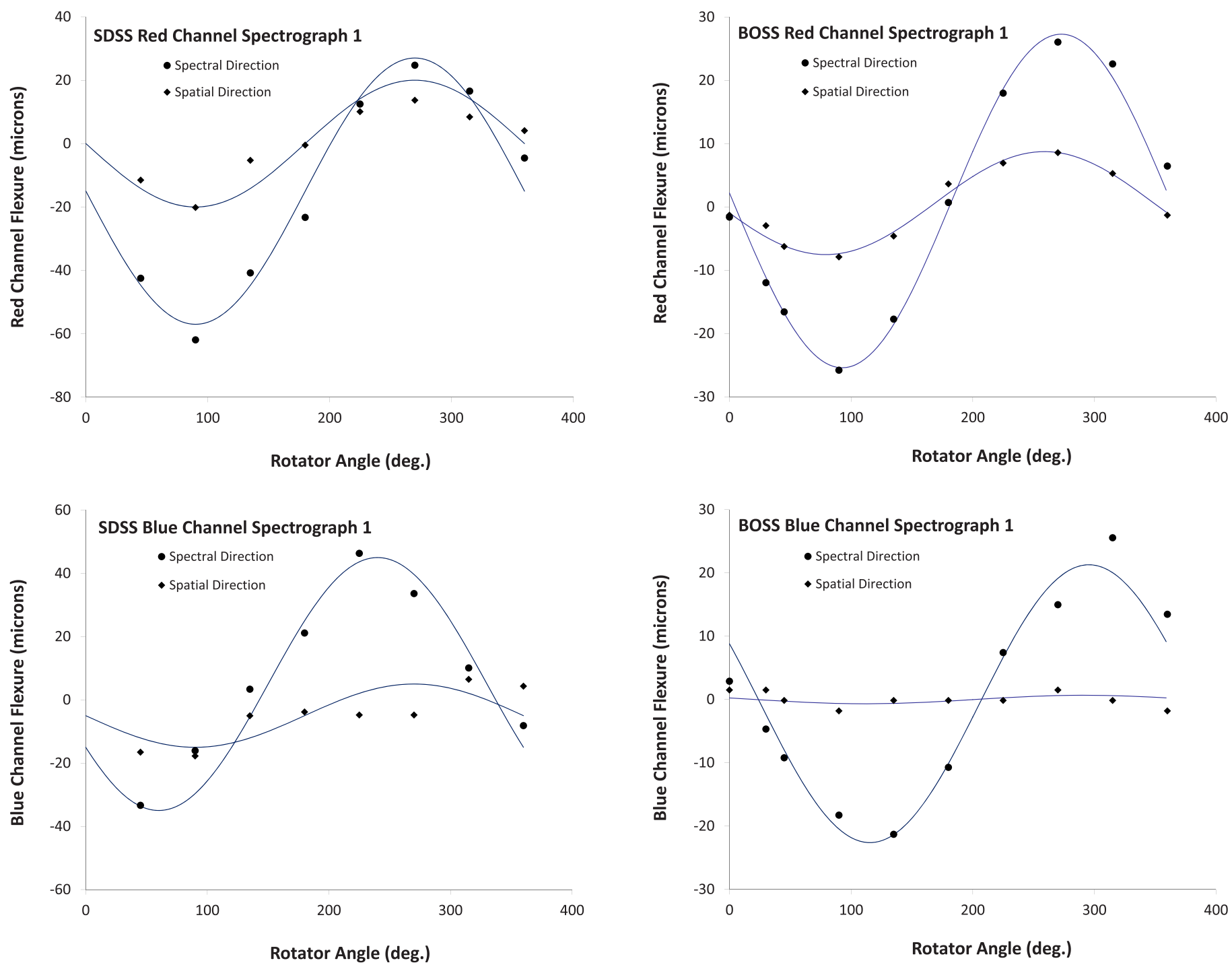

Figure 41. Gravity induced image motion in SDSS Spectrograph 1 as a function of rotator angle for a zenith angle of $72^{\circ} .24 \mu \mathrm{m}$ represents 1 pixel. The top and bottom plots show flexure in the red and blue channels, respectively. Flexure in the spectral direction is greater than in the spatial direction due to compliance in the collimator mount; a natural consequence of a tall mirror.

(A color version of this figure is available in the online journal.)

in the blue channel was reduced from $\sim 80 \mu$ m peak-to-valley to $\sim 47 \mu \mathrm{m}$ peak-to-valley in the spectral direction. However, given the smaller, $15 \mu \mathrm{m}$ pixel CCDs used in BOSS, the results are comparable to SDSS in units of pixels. For $15^{\circ}$ on the sky, the spectral shift is 0.41 pixels (worst case) in both channels of BOSS Spectrograph 1, modestly lower than the design expectation of 0.5 pixels; Spectrograph 2 is 0.44 pixels worst case. In the spatial direction, the worst case flexure is 0.18 pixels in Spectrograph 1, and 0.15 pixels in Spectrograph 2. Again, see Table 3 for a summary of the results.

\subsection{CCD and Electronics Performance}

We measured the CCD and electronics performance for SDSS and BOSS and report the performance in Table 4. Because the detectors are divided into multiple quadrants, we report the range of each measurement between the lowest value and highest value obtained in the quadrants of each detector. While only a few aspects of detector performance were specified during the design of the surveys, we describe the results of the detector

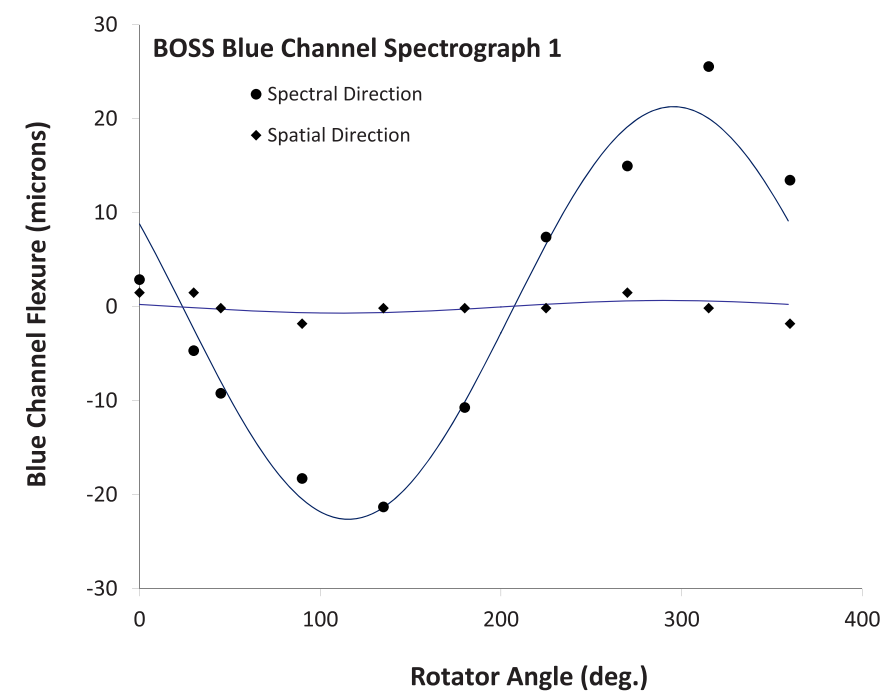

Figure 42. Gravity induced image motion in BOSS Spectrograph 1 as a function of rotator angle for a zenith angle of $72^{\circ} .15 \mu \mathrm{m}$ represents 1 pixel. The top and bottom plots show flexure in the red and blue channels, respectively.

(A color version of this figure is available in the online journal.)

performance below. When detector performance was specified, the CCDs exceeded specifications in all cases except for small regions on the $\mathrm{r} 2$ camera that slightly exceed the design goal of $2 e^{-}$pixel $^{-1} / 15$ minute of dark current.

First, the CCD images were checked for cosmetic defects such as saturated hot pixels, pixels with a large deviation from neighbors, blocked columns at low flux levels, and other bad pixels. These bad pixels are masked during the data processing. The hot pixels are recorded by identifying any pixel greater than 15,000 ADU in the bias exposures. Hot columns are identified in bias exposures as regions of connected pixels that lie above the background, in the direction of parallel clock transfer, by an amount equal to five times the read noise. For SDSS, pixel-to-pixel variation was mapped using the flat field dither technique described in Section 2.7. For BOSS, pixel-to-pixel variation is mapped using the images taken with the lossy-fiber as described in Section 3.7. Blocked columns and defective pixels are identified in these images as clustering of pixels that lie $50 \%$ below the mean background. The flat fields from these tests are also applied at the same time as the bad pixel masks in the 
Table 4

CCD Performance for SDSS and BOSS

\begin{tabular}{|c|c|c|c|c|c|}
\hline & $\begin{array}{l}\text { Read Noise } \\
\qquad\left(e^{-}\right)\end{array}$ & $\begin{array}{c}\text { Dark Current } \\
\left(e^{-} \text {pixel }^{-1} / 15 \text { minute }\right)\end{array}$ & $\begin{array}{c}\text { Gain } \\
\left(e^{-} \mathrm{ADU}^{-1}\right)\end{array}$ & $\begin{array}{c}\text { Number of } \\
\text { Bad Columns }\end{array}$ & $\begin{array}{l}\text { Fraction of } \\
\text { Bad Pixels }\end{array}$ \\
\hline \multicolumn{6}{|c|}{ SDSS } \\
\hline b1 & $2.8-3.8$ & $1.05-1.10$ & $1.05-1.10$ & 4 & \\
\hline b2 & $3.2-3.9$ & $1.23-1.26$ & $1.23-1.26$ & 4 & \\
\hline r1 & $3.5-4.1$ & $1.00-1.09$ & $1.00-1.09$ & 14 & \\
\hline $\mathrm{r} 2$ & $3.6-4.4$ & $1.04-1.05$ & 1.05 & 10 & \\
\hline \multicolumn{6}{|c|}{ Start of BOSS } \\
\hline b1 & $1.79-1.98$ & $0.64-0.68$ & $1.01-1.05$ & 0 & $2.0 \mathrm{e}-05$ \\
\hline b2 & $1.74-2.04$ & $0.61-0.65$ & 0.99-1.04 & 2 & $2.1 \mathrm{e}-04$ \\
\hline r1 & $2.36-2.72$ & $0.88-1.10$ & $1.54-1.97$ & 5 & $1.4 \mathrm{e}-04$ \\
\hline $\mathrm{r} 2$ & $2.42-2.99$ & $0.95-1.97$ & $1.54-1.96$ & 3 & $1.8 \mathrm{e}-04$ \\
\hline \multicolumn{6}{|c|}{ MJD 55300 (r2 replaced) } \\
\hline b1 & $1.83-2.01$ & $0.51-0.53$ & $1.01-1.05$ & 0 & $2.1 \mathrm{e}-05$ \\
\hline b2 & $1.87-2.03$ & $0.53-0.56$ & $0.99-1.04$ & 2 & $2.1 \mathrm{e}-04$ \\
\hline r1 & $2.43-2.73$ & $0.63-0.80$ & $1.54-1.97$ & 5 & $3.3 \mathrm{e}-04$ \\
\hline $\mathrm{r} 2$ & $2.73-2.89$ & $1.19-2.27$ & $1.59-1.66$ & 11 & $4.4 \mathrm{e}-04$ \\
\hline \multicolumn{6}{|c|}{ MJD 55800 (r1 replaced) } \\
\hline b1 & $1.77-2.02$ & $0.46-0.49$ & $1.01-1.05$ & 0 & $2.1 \mathrm{e}-05$ \\
\hline b2 & $1.86-2.01$ & $0.56-0.59$ & $0.99-1.04$ & 2 & $2.1 \mathrm{e}-04$ \\
\hline r1 & $2.45-2.82$ & $0.57-0.82$ & $1.47-1.93$ & 1 & $3.3 \mathrm{e}-04$ \\
\hline $\mathrm{r} 2$ & $2.85-2.88$ & $1.30-1.56$ & $1.59-1.66$ & 11 & $2.5 e-04$ \\
\hline
\end{tabular}

data processing. Other bad pixels are found from a combination of flats, biases, and dark frames.

In addition to cosmetic defects, the read noise, dark current, and gain of the CCDs were measured as shown in Table 4. The read noise was measured in a series of zero second exposures; bias frames that were obtained throughout the surveys. A series of 15 minute exposures is averaged pixel-by-pixel to determine the dark current in each pixel. We include any light source detected by the CCDs that does not originate from the fibers, including light leaks, in our measurement of dark current. The dark current in each quadrant of a CCD is averaged, and we report the minimum and maximum values of dark current in the four quadrants. Gain is determined for each quadrant from photon statistics in a series of flat field exposures.

For BOSS, we use measurements in the detector lab at LBNL to determine full-well depth for the red CCDs. We refer to documentation from $\mathrm{e} 2 \mathrm{v}$ for these measurements on the blue CCDs. The full-well depth exceeds the maximum number of electrons expected $\left(65,000 e^{-}\right.$pixel $\left.^{-1}\right)$ in a science exposure for all cameras. The charge transfer efficiency (CTE) records the fraction of electrons that are successfully transferred during each clock cycle during readout. CTE for the red CCDs was measured using an ${ }^{55} \mathrm{Fe}$ source (as described in Bebek et al. 2002; Dawson et al. 2008) in the LBNL detector lab for the red CCDs and recorded from the $2 \mathrm{v}$ documentation for the blue CCDs. All detectors exceed the CTE requirement of 0.99999 in both serial transfer and parallel transfer. Finally, to meet our goal for overall observing efficiency, a maximum of $70 \mathrm{~s}$ can be used for the CCD pre-exposure flush time and end-of-exposure readout time. The readout time of the BOSS CCDs has been measured to be $55.6 \mathrm{~s}$ as described above. This requirement means that not more than $10 \%$ of the observing time is used for the CCD flush and readout for two calibration exposures and four $900 \mathrm{~s}$ science exposures. Because the detectors have been replaced by newer technologies, we do not report values for full-well depth, CTE, or readout time for the SDSS detectors.

\section{CONCLUSION}

The SDSS spectrographs were designed to be highthroughput, robust instruments, to conduct an extragalactic survey producing a million redshifts over a five-year lifetime. These goals have clearly been met, with 1.6 million spectra of stars, galaxies, and quasars over 2880 plates, primarily in the northern Galactic cap at high Galactic latitudes. Spectra over $9274 \mathrm{deg}^{2}$ were collected in nine years of operation between the start of SDSS-I in 1999 and the completion of the first of two extended phases, SDSS-II, including SEGUE which produced roughly 250,000 stellar spectra between 2004-2008 (Yanny et al. 2009). These data were released to the astronomy community nearly every year; the full data set from SDSS-I and SDSS-II is included in DR8 (Aihara et al. 2011). Aside from a few minor technical issues where the instruments did not meet predicted design performance, the spectrographs proved to be quite reliable and exceptionally productive instruments. With improvements in CCD technology, the use of VPH gratings, and enhancements to the optics (i.e., fluid coupled camera triplets, new dichroics, and higher reflectivity collimator coatings) the instrumental throughput was enhanced significantly, allowing the use of smaller diameter fibers and making the BOSS survey possible. Along with improvements to the guide camera, modifications to improve flexure, and a host of minor upgrades, the BOSS spectrographs have proven to be as productive as the original SDSS design and have many years of life remaining.

The spectroscopic data from SDSS have enabled studies across a broad range of astronomical disciplines including the evolution and clustering of galaxies (e.g., Kauffmann et al. 2004; Tegmark et al. 2004), gravitational lensing (e.g., Bolton et al. 2006), the properties of quasars (e.g., Vanden Berk et al. 2001), and stellar astrophysics (e.g., West et al. 2008). One of the prominent scientific contributions from SDSS and SDSS-II data is the discovery of acoustic oscillation signatures in the clustering of galaxies (Eisenstein et al. 2005), opening the door 
to a new method of cosmological measurement. SDSS imaging and spectroscopic data have been included in more than 3500 refereed papers.

As described in detail in Dawson et al. (2013), the BOSS survey will cover $10,000 \mathrm{deg}^{2}$ in five years, obtaining spectra for 1.35 million luminous galaxies with redshifts $0.15<z<0.7$, and approximately 160,000 quasars with redshifts between $2.15<z<3.5$. The galaxies provide a highly biased tracer of matter, and at these densities over this area provide percentlevel distance scale measurements through studies of BAO. The quasars provide a backlight to illuminate neutral hydrogen through Ly $\alpha$ absorption along the line of sight, thereby mapping large-scale structure in the foreground of each quasar. These distance measurements will represent a significant improvement on the accuracy of existing BAO measurements, and will tighten the constraints on the dark energy equation of state, $w=p / \rho$, where $p$ is pressure and $\rho$ is density, and the evolution of $w$ with time. Detection of the BAO feature in quasar spectra would allow BOSS to make the first characterization of dark energy at early times, when dark energy should be sub-dominant according to the prevailing $\Lambda \mathrm{CDM}$ theory.

BOSS completed imaging of the southern Galactic cap spectroscopic footprint in 2008 and 2009; spectroscopic observations began 2009 December 5 after a three month commissioning phase. The algorithm for selecting galaxy targets is outlined briefly in White et al. (2011) and Eisenstein et al. (2011). The quasar target selection is described in Ross et al. (2012) using photometry from the SDSS imaging survey supplemented with data from programs at ultraviolet, infrared, and radio wavelengths to enhance the detection efficiency. These first two years of BOSS spectra include 324,198 unique galaxy targets $(z>0.43)$ with a $98.7 \%$ rate of successful classification, and 103,729 unique lower redshift galaxy targets $(0.15<z<0.43)$ with a $99.9 \%$ successful rate of classification. There were more than 85,000 quasars observed, 61,933 of which were at $z>2.15$. The spectra of these objects are classified as described in Bolton et al. (2012) and each quasar is visually inspected and recorded as explained in Pâris et al. (2012). Data from the first two years of the survey were included in DR9 (Ahn et al. 2012) and include the spectra from 831 plates.

The higher redshift sample of galaxies from DR9 has already been used to fit the position of the acoustic peak at an effective redshift $z=0.57$ (Anderson et al. 2012). With 1.7\% accuracy, this is the most precise distance constraint ever obtained from a galaxy survey at any redshift. The potential for distance measurements at $z>2$ using $\operatorname{Ly} \alpha$ forest absorption from quasars is promising; the first detection of flux correlations across widely separated sightlines was obtained in the first year of BOSS data to comoving separations of $60 \mathrm{~h}^{-1} \mathrm{Mpc}$ (Slosar et al. 2011). More recently, the acoustic peak was measured in the Ly $\alpha$ forest, two complimentary analyses produced distance measurements at $z>2$ with 3\% (Busca et al. 2013) and 2\% (Slosar et al. 2013) precision. BOSS is on pace to complete the $10,000 \mathrm{deg}^{2}$ spectroscopic footprint by the middle of 2014 , providing new cosmology constraints and a wealth of data for studies of galaxy evolution and quasar physics.

The optical spectrographs at APO will have unique capabilities for widefield, multiplexed spectroscopy well beyond the 2014 completion of the BOSS survey. The SDSS collaboration has proposed an "After SDSS III" (AS3) program for 2014-2020 that will capitalize on this resource. Four separate programs in AS3 will use the BOSS spectrograph, likely with only minor modifications. The extended BOSS (eBOSS) will probe dark energy and fundamental physics by making distance measurements with BAO in the redshift range $0.6<z<2$. The Time-Domain Spectroscopic Survey (TDSS) will obtain spectra of 50,000-100,000 Galactic and extragalactic variable sources with simultaneous, multi-epoch optical imaging data. In a program to follow up X-ray sources in new $0.28 \mathrm{keV}$ data obtained from the extended ROentgen Survey with an Imaging Telescope Array (eROSITA; Predehl et al. 2010), the SPectroscopic IDentification of eROSITA Sources (SPIDERS) survey will follow up 50,000-100,000 objects. Finally, Mapping Nearby Galaxies at Apache Point Observatory (MaNGA) will perform spatially resolved spectroscopy on approximately 10,000 nearby galaxies using 15 integral field units integrated into new BOSS-like cartridges. As with the original SDSS spectroscopic survey, these four surveys will provide a premier data sample for astrophysical studies from Galactic to cosmological scales.

Funding for SDSS-III has been provided by the Alfred P. Sloan Foundation, the Participating Institutions, the National Science Foundation, and the U.S. Department of Energy Office of Science. The SDSS-III Web site is http://www.sdss3.org/.

SDSS-III is managed by the Astrophysical Research Consortium for the Participating Institutions of the SDSS-III Collaboration including the University of Arizona, the Brazilian Participation Group, Brookhaven National Laboratory, University of Cambridge, Carnegie Mellon University, University of Florida, the French Participation Group, the German Participation Group, Harvard University, the Instituto de Astrofisica de Canarias, the Michigan State/Notre Dame/JINA Participation Group, Johns Hopkins University, Lawrence Berkeley National Laboratory, Max Planck Institute for Astrophysics, Max Planck Institute for Extraterrestrial Physics, New Mexico State University, New York University, Ohio State University, Pennsylvania State University, University of Portsmouth, Princeton University, the Spanish Participation Group, University of Tokyo, University of Utah, Vanderbilt University, University of Virginia, University of Washington, and Yale University.

\section{REFERENCES}

Abazajian, K. N., Adelman-McCarthy, J. K., Agüeros, M. A., et al. 2009, ApJS, 182,543

Adelman-McCarthy, J. K., Agüeros, M. A., Allam, S. S., et al. 2008, ApJS, 175,297

Ahn, C. P., Alexandroff, R., Allende Prieto, C., et al. 2012, ApJS, 203, 21

Aihara, H., Allende Prieto, C., An, D., et al. 2011, ApJS, 193, 29

Anderson, L., Aubourg, E., Bailey, S., et al. 2012, MNRAS, 427, 3435

Angel, J. R. P., Adams, M. T., Boroson, T. A., \& Moore, R. L. 1977, ApJ, 218,776

Bebek, C., Groom, D. E., Holland, S. E., et al. 2002, Proc. SPIE, 4669, 161

Bolton, A. S., Burles, S., Koopmans, L. V. E., Treu, T., \& Moustakas, L. A. 2006, ApJ, 638, 703

Bolton, A. S., Schlegel, D. J., Aubourg, É., et al. 2012, AJ, 144, 144

Burgh, E. B., Bershady, M. A., Westfall, K. B., \& Nordsieck, K. H. 2007, PASP, 119, 1069

Busca, N. G., Delubac, T., Rich, J., et al. 2013, A\&A, 552, A96

Clayton, C. A. 1989, A\&A, 213, 502

Cohen, J. G., Oke, J. B., Carr, M., Harris, F. H., \& Hamilton, D. 1988, in ASP Conf. Ser. 3, Fiber Optics in Astronomy, ed. S. C. Barden (San Francisco, CA: ASP), 190

Dawson, K., Bebek, C., Emes, J., et al. 2008, ITNS, 55, 1725

Dawson, K. S., Schlegel, D. J., Ahn, C. P., et al. 2013, AJ, 145, 10

Eisenstein, D. J., Annis, J., Gunn, J. E., et al. 2001, AJ, 122, 2267

Eisenstein, D. J., Weinberg, D. H., Agol, E., et al. 2011, AJ, 142, 72

Eisenstein, D. J., Zehavi, I., Hogg, D. W., et al. 2005, ApJ, 633, 560

Epps, H. W. 1990, Proc. SPIE, 1235, 550

Falco, E. E., Kurtz, M. J., Geller, M. J., et al. 1999, PASP, 111, 438 
Fukugita, M., Ichikawa, T., Gunn, J. E., et al. 1996, AJ, 111, 1748

Gunn, J. E., Carr, M., Rockosi, C., et al. 1998, AJ, 116, 3040

Gunn, J. E., Siegmund, W. A., Mannery, E. J., et al. 2006, AJ, 131, 2332

Heacox, W. D. 1986, AJ, 92, 219

Holland, S. E., Bebek, C. J., Dawson, K. S., et al. 2006, Proc. SPIE, 6276, 62760B

Huchra, J., Davis, M., Latham, D., \& Tonry, J. 1983, ApJS, 52, 89

Ivezić, Ž., Lupton, R. H., Schlegel, D., et al. 2004, AN, 325, 583

Kauffmann, G., White, S. D. M., Heckman, T. M., et al. 2004, MNRAS, 353,713

Kobulnicky, H. A., Nordsieck, K. H., Burgh, E. B., et al. 2003, Proc. SPIE, 4841,1634

Murphy, J. D., Hill, G. J., MacQueen, P. J., et al. 2012, Proc. SPIE, 8446, 84465F Nordsieck, K., Nosan, F., \& Schier, J. A. 2010, Proc. SPIE, 7735, 773582

Padmanabhan, N., Schlegel, D. J., Finkbeiner, D. P., et al. 2008, ApJ, 674,1217

Pâris, I., Petitjean, P., Aubourg, É., et al. 2012, A\&A, 548, A66

Pier, J. R., Munn, J. A., Hindsley, R. B., et al. 2003, AJ, 125, 1559
Predehl, P., Andritschke, R., Böhringer, H., et al. 2010, Proc. SPIE, 7732 , $77320 \mathrm{U}$

Ross, N. P., Myers, A. D., Sheldon, E. S., et al. 2012, ApJS, 199, 3

Schlegel, D., White, M., \& Eisenstein, D. 2009, Astro2010: The Astronomy and Astrophysics Decadal Survey, Science White Papers, 314

Slosar, A., Font-Ribera, A., Pieri, M. M., et al. 2011, JCAP, 09, 001

Slosar, A., Iršič, V., Kirkby, D., et al. 2013, JCAP, 04, 026

Smith, J. A., Tucker, D. L., Kent, S., et al. 2002, AJ, 123, 2121

Tegmark, M., Blanton, M. R., Strauss, M. A., et al. 2004, ApJ, 606, 702

Tucker, D. L., Kent, S., Richmond, M. W., et al. 2006, AN, 327, 821

Vanden Berk, D. E., Richards, G. T., Bauer, A., et al. 2001, AJ, 122, 549

Weinberg, D. H., Mortonson, M. J., Eisenstein, D. J., et al. 2013, PhR, in press (arXiv:1201.2434)

West, A. A., Hawley, S. L., Bochanski, J. J., et al. 2008, AJ, 135, 785

White, M., Blanton, M., Bolton, A., et al. 2011, ApJ, 728, 126

Wilson, R. N. 1999, Reflecting Telescope Optics II (Berlin: Springer)

Yanny, B., Rockosi, C., Newberg, H. J., et al. 2009, AJ, 137, 4377

York, D. G., Adelman, J., Anderson, J. E., Jr., et al. 2000, AJ, 120, 1579 